\title{
Dioxygen Reduction by a Pd(0)-Hydroquinone Diphosphine Complex
}

\author{
Kyle T. Horak, Theodor Agapie* \\ *To whom correspondence should be addressed. E-mail: agapie@caltech.edu. \\ Department of Chemistry and Chemical Engineering, California Institute of Technology, $1200 \mathrm{E}$. \\ California Blvd. MC 127-72, Pasadena, CA, USA. \\ Supporting Online Material
}

Contents

Experimental Details

General Considerations

Synthesis of 2,5-diiodo-1,4-bis(methoxymethylether)hydroquinone (A)

Synthesis of 2,5-bis(2-bromophenyl)-1,4-(methoxymethylether)hydroquinone (B) 5

Synthesis of 2,5-bis(2-(diisopropylphosphino)phenyl)-1,4-hydroquinone (1-H) 5

Synthesis of Compound 2

Synthesis of Complex $\mathbf{3}$ using $\mathrm{Pd}(\mathrm{OAc})_{2}$

Synthesis of Compound 3 using $\mathrm{Pd}_{2}(\mathrm{dba})_{3}$ and $\mathrm{O}_{2}$

Synthesis of Compound 4-H

Synthesis of Compound 5-H

Synthesis of Compound 6-H

Synthesis of Compound 7-H

Synthesis of Compound 4-Me

Synthesis of Compound 6-Me

In Situ Preparation of Compound 8-Me

Synthesis of Compound (3) $\mathbf{H}^{+}$

NMR Spectra

Figure S1. ${ }^{1} \mathrm{H}-\mathrm{NMR}\left(500 \mathrm{MHz}, \mathrm{CDCl}_{3}\right)$ spectrum of $\mathbf{A} . \quad 10$

Figure S2. ${ }^{1} \mathrm{H}-\mathrm{NMR}\left(500 \mathrm{MHz}, \mathrm{CDCl}_{3}\right)$ spectrum of $\mathbf{B}$. 11

Figure S3. ${ }^{13} \mathrm{C}-\mathrm{NMR}\left(126 \mathrm{MHz}, \mathrm{CDCl}_{3}\right)$ spectrum of $\mathbf{B}$. 11

Figure S4. ${ }^{1} \mathrm{H}-\mathrm{NMR}\left(300 \mathrm{MHz}, \mathrm{C}_{6} \mathrm{D}_{6}\right)$ spectrum of $\mathbf{1}-\mathbf{H}$.

Figure S5. ${ }^{31} \mathrm{P}-\mathrm{NMR}\left(121 \mathrm{MHz}, \mathrm{C}_{6} \mathrm{D}_{6}\right)$ spectrum of $\mathbf{1 - H} . \quad 12$

Figure S6. ${ }^{13} \mathrm{C}-\mathrm{NMR}\left(126 \mathrm{MHz}, \mathrm{C}_{6} \mathrm{D}_{6}\right)$ spectrum of $\mathbf{1 - H} . \quad 12$

Figure S7. ${ }^{1} \mathrm{H}-\mathrm{NMR}\left(500 \mathrm{MHz}, \mathrm{CD}_{2} \mathrm{Cl}_{2}\right)$ spectrum of $2 . \quad 12$

Figure S8. ${ }^{31} \mathrm{P}-\mathrm{NMR}\left(121 \mathrm{MHz}, \mathrm{CD}_{2} \mathrm{Cl}_{2}\right)$ spectrum of $2 . \quad 13$

Figure S9. ${ }^{13} \mathrm{C}-\mathrm{NMR}\left(126 \mathrm{MHz}, \mathrm{CD}_{2} \mathrm{Cl}_{2}\right)$ spectrum of $2 . \quad 13$

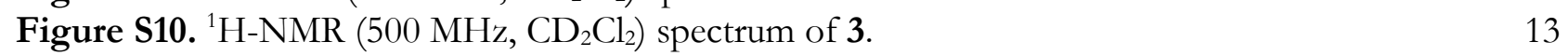

Figure S11. ${ }^{31} \mathrm{P}-\mathrm{NMR}\left(121 \mathrm{MHz}, \mathrm{CD}_{2} \mathrm{Cl}_{2}\right)$ spectrum of $\mathbf{3 .} 14$

Figure S12. ${ }^{13} \mathrm{C}-\mathrm{NMR}\left(126 \mathrm{MHz}, \mathrm{CD}_{2} \mathrm{Cl}_{2}\right)$ spectrum of $\mathbf{3} .14$

Figure S13. ${ }^{1} \mathrm{H}-\mathrm{NMR}\left(500 \mathrm{MHz}, \mathrm{C}_{6} \mathrm{D}_{6}\right)$ spectrum of 4-H. 14

Figure S14. ${ }^{31} \mathrm{P}-\mathrm{NMR}\left(121 \mathrm{MHz}, \mathrm{C}_{6} \mathrm{D}_{6}\right)$ spectrum of 4-H. $\quad 15$

Figure S15. ${ }^{13} \mathrm{C}-\mathrm{NMR}\left(126 \mathrm{MHz}, \mathrm{C}_{6} \mathrm{D}_{6}\right)$ spectrum of 4-H. $\quad 15$

Figure S16. ${ }^{1} \mathrm{H}-\mathrm{NMR}\left(500 \mathrm{MHz}, \mathrm{CD}_{3} \mathrm{CN}\right)$ spectrum of 5-H.

Figure S17. ${ }^{31} \mathrm{P}-\mathrm{NMR}\left(121 \mathrm{MHz}, \mathrm{CD}_{3} \mathrm{CN}\right)$ spectrum of 5-H. 16 
Figure S18. ${ }^{13} \mathrm{C}-\mathrm{NMR}\left(126 \mathrm{MHz}, \mathrm{CD}_{3} \mathrm{CN}\right)$ spectrum of $\mathbf{5}-\mathbf{H}$.

Figure S19. ${ }^{19} \mathrm{~F}-\mathrm{NMR}\left(282 \mathrm{MHz}, \mathrm{CD}_{3} \mathrm{CN}\right)$ spectrum of $\mathbf{5 - H} . \quad 16$

Figure S20. ${ }^{1} \mathrm{H}-\mathrm{NMR}\left(500 \mathrm{MHz}, \mathrm{CD}_{3} \mathrm{CN}\right)$ spectrum of $\mathbf{6 - H} . \quad 17$

Figure S21. ${ }^{31} \mathrm{P}-\mathrm{NMR}\left(121 \mathrm{MHz}, \mathrm{CD}_{3} \mathrm{CN}\right)$ spectrum of $\mathbf{6 - H} . \quad 17$

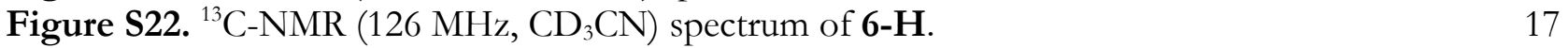

Figure S23. ${ }^{19} \mathrm{~F}-\mathrm{NMR}\left(282 \mathrm{MHz}, \mathrm{CD}_{3} \mathrm{CN}\right)$ spectrum of $\mathbf{6 - H} . \quad 18$

Figure S24. ${ }^{1} \mathrm{H}-\mathrm{NMR}\left(500 \mathrm{MHz}, \mathrm{CD}_{3} \mathrm{CN}\right)$ spectrum of 7-H. 18

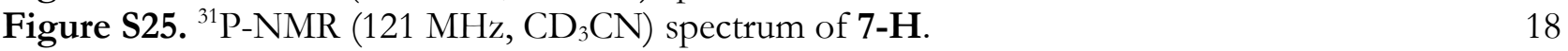

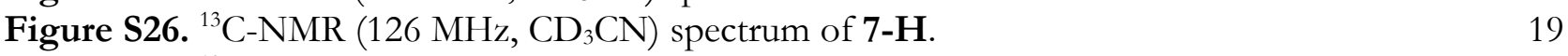

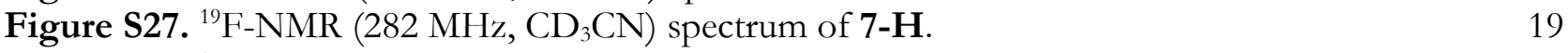

Figure S28. ${ }^{1} \mathrm{H}-\mathrm{NMR}\left(500 \mathrm{MHz}, \mathrm{C}_{6} \mathrm{D}_{6}\right)$ spectrum of 4-Me. 19

Figure S29. ${ }^{31} \mathrm{P}-\mathrm{NMR}\left(121 \mathrm{MHz}, \mathrm{C}_{6} \mathrm{D}_{6}\right)$ spectrum of 4-Me. 20

Figure S30. ${ }^{13} \mathrm{C}-\mathrm{NMR}\left(126 \mathrm{MHz}, \mathrm{C}_{6} \mathrm{D}_{6}\right)$ spectrum of 4-Me. 20

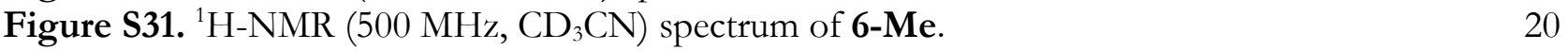

Figure S32. ${ }^{31} \mathrm{P}-\mathrm{NMR}\left(121 \mathrm{MHz}, \mathrm{CD}_{3} \mathrm{CN}\right)$ spectrum of 6-Me. 21

Figure S33. ${ }^{13} \mathrm{C}-\mathrm{NMR}\left(126 \mathrm{MHz}, \mathrm{CD}_{3} \mathrm{CN}\right)$ spectrum of 6-Me. $\quad 21$

Figure S34. ${ }^{19} \mathrm{~F}-\mathrm{NMR}\left(282 \mathrm{MHz}, \mathrm{CD}_{3} \mathrm{CN}\right)$ spectrum of 6-Me. 21

Figure S35. ${ }^{1} \mathrm{H}-\mathrm{NMR}\left(500 \mathrm{MHz}, \mathrm{d}_{8}-\mathrm{THF}\right)$ spectrum of 8-Me. 22

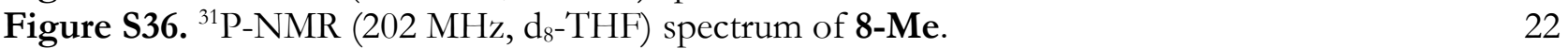

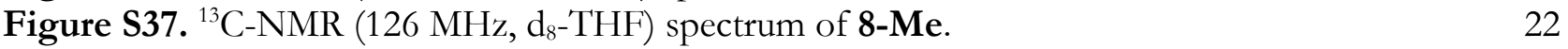

Figure S38. ${ }^{1} \mathrm{H}-\mathrm{NMR}\left(300 \mathrm{MHz}, \mathrm{CD}_{3} \mathrm{CN}\right)$ spectrum of $\mathbf{( 3 ) \mathbf { H } ^ { + } .} 23$

Figure S39. ${ }^{31} \mathrm{P}-\mathrm{NMR}\left(202 \mathrm{MHz}, \mathrm{CD}_{3} \mathrm{CN}\right)$ spectrum of $\mathbf{( 3 ) \mathbf { H } ^ { + } .} 23$

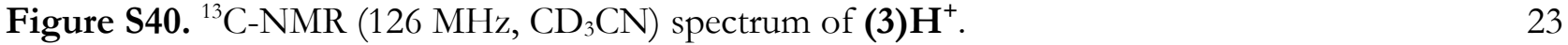

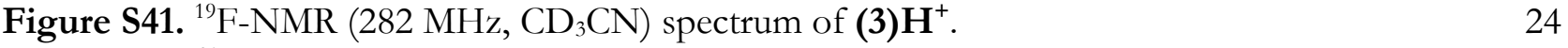

Figure S42. ${ }^{31} \mathrm{P}-\mathrm{NMR}$ Comparison of $(\mathbf{3}) \mathbf{H}^{+}$produced from $\mathbf{3}$ or $\mathbf{6 - H}$

Volumetric Measurement of $\mathrm{O}_{2}$ Consumption

Toepler Pump Experimental Details 25

Table S1. Results of volumetric $\mathrm{O}_{2}$ measurements. 25

ReactIR Spectra

Figure S43. Solution ReactIR spectrum for $4-\mathrm{H}$ at $-78^{\circ} \mathrm{C}$ before $\mathrm{O}_{2}$ addition. 26

Figure S44. Solution ReactIR spectrum for $4-\mathbf{H}$ at $-78{ }^{\circ} \mathrm{C}$ after $\mathrm{O}_{2}$ addition. 26

Figure S45. Solution ReactIR spectrum for 4-H after warming to $-35^{\circ} \mathrm{C}$ after $\mathrm{O}_{2}$ addition. $\quad 27$

Figure S46. Solution ReactIR spectrum for 4-H after warming to $25^{\circ} \mathrm{C}$ after $\mathrm{O}_{2}$ addition. 27

UV/Vis Spectra

Figure S47. Solution UV/Vis spectrum for 2 addition. 28

$\begin{array}{ll}\text { Figure S48. Solution UV/Vis spectrum for } 3 \text { addition. } & 28\end{array}$

Figure S49. Solution UV/Vis spectrum for $\mathbf{6 - H}$ at $25{ }^{\circ} \mathrm{C}$ following $\mathrm{O}_{2}$ addition. 29

Figure S50. Solution UV/Vis spectrum for 6-H after conversion to Int 3 at $25^{\circ} \mathrm{C}$. 29

Oxidation of $\mathbf{4 - H}$ to $\mathbf{3}$ by Various Substrates

Dioxygen 29

$\begin{array}{lr}\text { Nitric Oxide } & 30\end{array}$

$\begin{array}{ll}\text { Nitrous Oxide } & 30\end{array}$

2,4,6-Tritertbutyl Phenoxyl Radical 30

1-Azido Adamantane $\quad 30$

$\begin{array}{lr}\text { Trimethylamine } n \text {-oxide } & 30\end{array}$ 
Representative Conditions for Testing Substrate Oxidation by 8 -Me

Para-trifluoromethyl benzyl alcohol

Para-trifluoromethyl-benzaldehyde

Cyclohexane Carboxaldehyde

Cyclohexene

Triphenylphosphine

Figure S51. NMR spectra of $\mathrm{PPh}_{3}$ addition to 8-Me at $-78^{\circ} \mathrm{C}$ in THF.

Cyclic Voltammetry Data

Electrochemical Details

Figure S52. CV data for complex 2.

Figure S53. CV data for complex 3.

Table S2. Crystal and refinement data for reported complexes. 


\section{Experimental Section}

\section{General Considerations}

All air- and/or water-sensitive compounds were manipulated using standard vacuum or Schlenk line techniques or in an inert atmosphere glove box. The solvents for air- and moisture-sensitive reactions were dried over sodium benzophenone ketyl, calcium hydride, or by the method of Grubbs. ${ }^{1}$ All NMR solvents were purchased from Cambridge Isotopes Laboratories, Inc. and dried over sodium benzophenone ketyl or calcium hydride. Unless mentioned otherwise, reagents were used as received from commercial suppliers without further purification. Nickel(II)acetate, palladium(II)acetate, chlorodiisopropylphosphine, boron tribromide, silver trifluoromethanesulfonate, $1.7 \mathrm{M} t \mathrm{BuLi}$ in pentane, diisopropylethylamine, 1,4-dimethoxybenzene, were purchased from Sigma Aldrich. (1,5cyclooctadiene)palladium(II)chloride, $\quad$ bis(1,5-cyclooctadiene)-Ni(0), $\quad$ tetrakis(triphenylphosphine)palladium(0), tris-(dibenzylideneacetone)-dipalladium $(0), \quad$ were purchased from Strem Chemicals Inc.Iodine monochloride was purchased from Alfa Aesar. , 2-Bromophenylboronic acid was purchased from Ark Pharm. 2.1 M methyl chloromethyl ether $(\mathrm{MOMCl})$ in toluene, ${ }^{2}$ (2,2'-bipyridine)(1,5-cyclooctadiene)nickel(0), ${ }^{3}$ 1,4-dimethoxy2,5-diiodobenzene, ${ }^{4}$ 2,5-diiodo-1,4-hydroquinone, ${ }^{5}$ and $\mathbf{1}-\mathbf{M e}^{6}$ were made according to literature procedures. All ${ }^{1} \mathrm{H},{ }^{13} \mathrm{C}$, and ${ }^{31} \mathrm{P}$ spectra were recorded on Varian Mercury 300 $\mathrm{MHz}$, or Varian INOVA-500 or $600 \mathrm{MHz}$ spectrometers at room temperature. Chemical shifts for ${ }^{1} \mathrm{H}$ and ${ }^{13} \mathrm{C}$ NMR data are reported relative to residual solvent peaks. ${ }^{7}{ }^{31} \mathrm{P}$ NMR chemical shifts are reported with respect to the deuterated solvent used to lock the instrument. Solution IR spectra were obtained as solution samples using a $\mathrm{CaF}_{2}$ window cell on a Thermo Scientific Nicolet 6700 FT-IR spectrometer. In situ solution IR spectra were recorded on an iC10 ReactIR (Mettler Toledo) equipped with a K4 conduit and a SiComp Sentinel sensor in a custom-made glass vessel. The UV/Vis spectra were recorded on a Varian Cary Bio 50 spectrophotometer. Low-temperatured UV/Vis spectra were obtained using a Varian dip-probe (661.202-UV, $10 \mathrm{~mm})$ and custom-made glass vessel. Elemental analyses were performed by Robertson Microlit Laboratories, Ledgewood, NJ.

\section{Synthetic Scheme for 2,5-bis(2-diisopropylphosphinophenyl)-1,4-hydroquinone (1-H)}

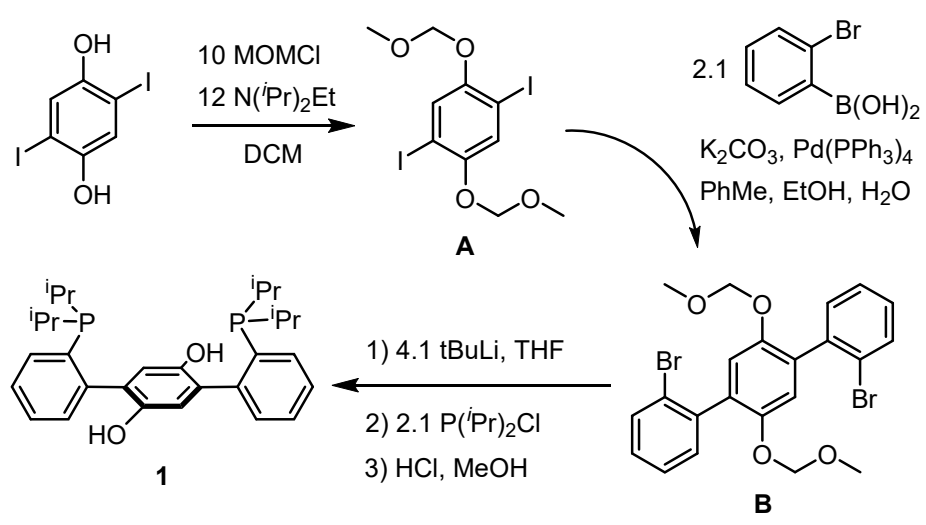

\section{Synthesis of 2,5-diiodo-1,4-bis(methoxymethylether)hydroquinone (A)}

The synthesis of 2,5-diiodo-1,4-bis(methoxymethylether)hydroquinone has been previously reported, however this protocol provides an alternative synthetic route. 2,5diiodo-1,4-hydroquinone ( $2 \mathrm{~g}, 5.53 \mathrm{mmol}, 1$ equiv) was added to a 3 -neck flask under a 
counterflow of nitrogen. Degassed, anhydrous dichloromethane $(100 \mathrm{~mL})$ was then added with a cannula to yield a suspension. Diisopropylethylamine (11.6 mL, $66.3 \mathrm{mmol}, 12$ equiv) was then added with a syringe and the reaction mixture allowed to stir for 1 minute. Methylchloromethylether was then added ( $2.1 \mathrm{M}$ in PhMe, $26.3 \mathrm{~mL}, 55.3 \mathrm{mmol}, 10$ equiv) with a syringe and the reaction was allowed to stir for 3-4 hours during which time the solution became homogenous. The reaction was monitored by GC-MS until all starting material was consumed. The reaction mixture was then washed with a saturated sodium bicarbonate solution. The organic layer was then collected and dried with magnesium sulfate before volatiles were removed by rotary evaporation to yield the clean product as an offwhite residue. Characterization matched previous literature reports. Yield: $2.07 \mathrm{~g}(83 \%)$.

\section{Synthesis of 2,5-bis(2-bromophenyl)-1,4-(methoxymethylether)hydroquinone (B)}

The Suzuki coupling to produce the terphenyl product was run as a modification of literature procedure. ${ }^{8}$ A Schlenk tube fitted with a Teflon stopper was charged with 2,5diiodo-1,4-bis(methoxymethylether)hydroquinone (500 mg, $1.11 \mathrm{mmol}, 1$ equiv.), 2-bromophenylboronic acid (468.6 mg, $2.33 \mathrm{mmol}, 2.1$ equiv.), and $\mathrm{K}_{2} \mathrm{CO}_{3}$ (921.3 mg, $6.67 \mathrm{mmol}, 6$ equiv.). Toluene $(26 \mathrm{~mL})$, ethanol $(6 \mathrm{~mL})$, and water $(6 \mathrm{~mL})$ were then transferred to the Schlenk tube along with a magnetic stirbar. The mixture was degassed by two freeze pump thaw cycles and then put under positive nitrogen pressure. Under a strong counterflow of nitrogen, $\mathrm{Pd}\left(\mathrm{PPh}_{3}\right)_{4}(64.2 \mathrm{mg}, 0.056 \mathrm{mmol}, 0.05$ equiv.) was added and the solution became a pale yellow color. The reaction mixture was then heated to $65^{\circ} \mathrm{C}$ and stirred for $16-24 \mathrm{hrs}$ while monitoring the reaction by GC-MS. The volatiles were then removed on a rotovap and the residue extracted using dichloromethane and water. The organic layer was then collected and dried using magnesium sulfate before volatiles were removed on a rotovap. Following recrystallation from dichloromethane-methanol the product was obtained as off-white crystalline powder. Yield: $400 \mathrm{mg}(71 \%) .{ }^{1} \mathrm{H}$ NMR $\left(500 \mathrm{MHz}, \mathrm{CDCl}_{3}\right) \delta 7.67\left(\mathrm{~d}, \mathrm{~J}_{\mathrm{HH}}=8.0\right.$ $\mathrm{Hz}, \mathrm{ArH}, 2 \mathrm{H}$ ), 7.37 (m, ArH, 4H), 7.25 - 7.20 (m, $\operatorname{ArH}, 2 \mathrm{H}), 7.06$ (s, central-ArH, 2H), 5.02 (broad s, $\left.\mathrm{OCH}_{2} \mathrm{O}, 4 \mathrm{H}\right), 3.33\left(\mathrm{~s}, \mathrm{OCH}_{3}, 6 \mathrm{H}\right) \cdot{ }^{13} \mathrm{C} \mathrm{NMR}\left(126 \mathrm{MHz}, \mathrm{CDCl}_{3}\right) \delta 149.23$ (s), 139.54 (s), 132.68 (s), 132.17 (broad s), 131.93 (s), 129.00 (s), 127.11 (s), 124.21 (s), 118.74 (s), 96.14 (s), 56.20 (s). GC-MS (m/z): Calcd, $508.0\left(\mathrm{M}^{+}\right)$. Found: $508.0\left(\mathrm{M}^{+}\right)$, FAB-MS $(\mathrm{m} / \mathrm{z})$ : Calcd, $506.9630(\mathrm{M}+)$. Found: $506.9623\left(\mathrm{M}^{+}\right)$.

\section{Synthesis of 2,5-bis(2-(diisopropylphosphino)phenyl)-1,4-hydroquinone (1-H)}

Phosphination run as a modification of literature procedures. A Schlenk tube fitted with a screw-in Telfon stopper was charged with 2,5-bis(2-bromophenyl)-1,4bis(methoxymethylether)hydroquinone (1 g, $1.96 \mathrm{mmol}, 1$ equiv.) and a magnetic stirbar. Tetrahydrofuran $(60 \mathrm{~mL})$ was then to the Schlenk tube. The reaction was cooled to $-78{ }^{\circ} \mathrm{C}$ and tert-butyllithium (1.7 M pentane solution, $5.4 \mathrm{~mL}, 8.07 \mathrm{mmol}, 4.1$ equiv.) was added while stirring to generate a pale yellow solution. The reaction mixture was allowed to warm to room temperature and then stirred for an additional hour. During this time the solution became a cloudy suspension. Chlorodiisopropyl phosphine $(0.656 \mathrm{~mL}, 4.13 \mathrm{mmol}, 2.1$ equiv.) was then added to the reaction via syringe. The solution immediately became a homogenous pale yellow solution which was allowed to stir for 16 hours. The volatiles were then removed under reduced pressure on the Schlenk line. Acidified methanol $(13 \mathrm{~mL}$ concentrated $\mathrm{HCl}, 60 \mathrm{~mL} \mathrm{MeOH}$ ) was quickly degassed on the Schlenk line and then cannula transferred to the dried reaction residue which formed a pale yellow homogeneous solution. The reaction mixture was allowed to stir for $4 \mathrm{hrs}$ at $70{ }^{\circ} \mathrm{C}$ to ensure complete removal of the methoxymethylether protecting group before all volatiles were removed 
under reduced pressure. The reaction was then brought into a wet inert atmosphere glovebox and extracted with dichloromethane. The organic layer was washed with saturated aqueous potassium carbonate followed by saturated ammonium chloride. The organic layer was then dried over magnesium sulfate and the volatiles were then removed under reduced pressure. After drying the residue overnight with heating to $60{ }^{\circ} \mathrm{C}$ the reaction was then brought into the dry inert atmosphere glovebox. The residue was washed with cold pentanes and then cold ether to yield the pure product as an off-white solid. Yield: $460 \mathrm{mg}(47 \%) .{ }^{1} \mathrm{H}$ NMR spectra of product is broad at room temperature due to hindered rotation around aryl-aryl bonds. This is corroborated by ${ }^{31} \mathrm{P}$ NMR where two distinct peaks are observed at room temperature. ${ }^{1} \mathrm{H}$ NMR $\left(300 \mathrm{MHz}, \mathrm{C}_{6} \mathrm{D}_{6}\right) \delta 7.22$ (broad s, $\left.\mathrm{ArH}, 4 \mathrm{H}\right), 7.05$ (broad s, ArH, 4H), 5.78 (broad s, OH, 1H), 5.42 (broad s, OH, 1H), 1.93 (broad s, CH, 2H), 1.71 (broad s, CH, 2H), 0.84 (unresolved $\left.\mathrm{m}, \mathrm{CH}_{3}, 24 \mathrm{H}\right) .{ }^{13} \mathrm{C}\left\{{ }^{1} \mathrm{H}\right\}$ NMR $\left(126 \mathrm{MHz}, \mathrm{C}_{6} \mathrm{D}_{6}\right) \delta$ 145.76 (s), 134.38 (d, JPC = $45.8 \mathrm{~Hz}), 131.94$ (s), 129.24 (s), 127.01 (s), 121.76 (s), 120.72 (s), 25.35 (s), 21.97 (s), 21.33 (s), 19.89 (s), 19.27 (s), 18.18 (s). At room temperature, 1 shows two distinct ${ }^{31} \mathrm{P}$ NMR signals due to hindered rotation around the aryl-aryl bonds. ${ }^{31} \mathrm{P}\left\{{ }^{1} \mathrm{H}\right\}$ NMR (121 MHz, C6 $\left.\mathrm{D}_{6}\right) \delta 1.80$ (s), -0.06 (s). FAB-MS (m/z): Calcd: 495.2578 (M+). Found: $495.2582\left(\mathrm{M}^{+}\right)$.

\section{Synthesis of Compound 2}

Compound 1-H (100 mg, $0.202 \mathrm{mmol}, 1$ equiv.) was dissolved in tetrahydrofuran (6 $\mathrm{mL})$ and transferred to a Schlenk tube fitted with a screw-in Teflon stopper. $\mathrm{Ni}(\mathrm{OAc})_{2}(35.7$ $\mathrm{mg}, 0.202 \mathrm{mmol}, 1$ equiv.) was suspended in tetrahydrofuran and then added to the solution of 1. The Schlenk tube was then sealed and heated to $80{ }^{\circ} \mathrm{C}$ for 16 hours outside the glovebox during which time to solution became a cloudy green. The reaction mixture was then dried under reduced pressure and brought back into the inert atmosphere glovebox. The crude residue was suspended in diethyl ether and filtered onto a Celite pad. The green solid was washed with additional diethyl ether until the washes became colorless. The product was brought through the Celite pad using tetrahydrofuran. The combined tetrahydrofuran filtrate was dried under reduced pressure to yield the clean product as a green powder. Yield: $65 \mathrm{mg}(58 \%) .{ }^{1} \mathrm{H}$ NMR $\left(500 \mathrm{MHz}, \mathrm{CD}_{2} \mathrm{Cl}_{2}\right) \delta 7.62\left(\mathrm{~d}, \mathrm{~J}_{\mathrm{HH}}=7.6 \mathrm{~Hz}\right.$, $\operatorname{ArH}, 2 \mathrm{H}), 7.58\left(\mathrm{t}, \mathrm{J}_{\mathrm{HH}}=7.4 \mathrm{~Hz}, \operatorname{ArH}, 2 \mathrm{H}\right), 7.50\left(\mathrm{t}, \mathrm{J}_{\mathrm{HH}}=6.7 \mathrm{~Hz}, \operatorname{ArH}, 2 \mathrm{H}\right), 7.45\left(\mathrm{t}, \mathrm{J}_{\mathrm{HH}}=7.5\right.$ $\mathrm{Hz}, \mathrm{ArH}, 2 \mathrm{H}), 6.16$ (s, central ArH, 2H), 2.56 (m, CH, 2H), 2.28 (m, CH, 2H), $1.32-1.24$ $\left(\mathrm{m}, \mathrm{CH}_{3}, 12 \mathrm{H}\right), 1.21\left(\mathrm{dd}, \mathrm{J}_{\mathrm{PH}}=16.1 \mathrm{~Hz}, \mathrm{~J}_{\mathrm{HH}}=7.0 \mathrm{~Hz}, \mathrm{CH}_{3}, 6 \mathrm{H}\right), 1.09\left(\mathrm{dd}, \mathrm{J}_{\mathrm{PH}}=15.8 \mathrm{~Hz}, \mathrm{~J}_{\mathrm{HH}}\right.$ $\left.=7.0 \mathrm{~Hz}, \mathrm{CH}_{3}, 6 \mathrm{H}\right) \cdot{ }^{13} \mathrm{C}\left\{{ }^{1} \mathrm{H}\right\} \mathrm{NMR}\left(126 \mathrm{MHz}, \mathrm{CD}_{2} \mathrm{Cl}_{2}\right) \delta 162.79(\mathrm{~s}), 146.43-145.62(\mathrm{~m})$, $138.53-136.77(\mathrm{~m}), 130.95\left(\mathrm{~d}, \mathrm{~J}_{\mathrm{PC}}=16.1 \mathrm{~Hz}\right), 127.68(\mathrm{~s}), 120.33-117.76(\mathrm{~m}), 96.46(\mathrm{~s})$, 27.29 (m), 19.99 (s), 17.97 (s), 17.82 (s), 17.43 (s). ${ }^{31} \mathrm{P}\left\{{ }^{1} \mathrm{H}\right\}$ NMR (121 MHz, $\left.\mathrm{CD}_{2} \mathrm{Cl}_{2}\right) \delta 54.30$ (s). IR $\left(\mathrm{CaF}_{2}\right.$ window, THF, $\left.\mathrm{cm}^{-1}\right) v_{\mathrm{C}-\mathrm{o}}$ : 1597. Anal. Calcd. for: $\mathrm{C}_{30} \mathrm{H}_{38} \mathrm{NiO}_{2}(2)(\%): \mathrm{C}, 65.36$; $\mathrm{H}$, 6.95. $\mathrm{C}_{31} \mathrm{H}_{40} \mathrm{Cl}_{2} \mathrm{NiO}_{2}(2 \bullet \mathbf{D C M})(\%)$ : C, 58.53; H, 6.34. Found: C, 58.94; H, 6.18.

\section{Synthesis of Complex 3 using $\operatorname{Pd}(\mathrm{OAc})_{2}$}

Compound 1-H (100mg, $0.202 \mathrm{mmol}, 1$ equiv.) was dissolved in tetrahydrofuran (10 $\mathrm{mL}$ ) and transferred to a $20 \mathrm{~mL}$ scintillation vial. Palladium diacetate $(45.4 \mathrm{mg}, 0.202 \mathrm{mmol}$, 1 equiv.) was added as a tetrahydrofuran solution, which in the immediate formation of a deep purple solution. The reaction mixture was allowed to stir for 16 hours before volatiles were removed under reduced pressure to yield a purple residue. The residue was filtered through a Celite pad with tetrahydrofuran. The combined tetrahydrofuran filtrate was dried under reduced pressure to yield the product as a purple powder. Yield: $70 \mathrm{mg}(58 \%)$. 


\section{Synthesis of Compound 3 using $\mathbf{P d}_{2}(\mathrm{dba})_{3}$ and $\mathrm{O}_{2}$}

Compound 1-H (300 mg, $0.606 \mathrm{mmol}, 1$ equiv.) was dissolved in tetrahydrofuran (10 $\mathrm{mL}$ ) and then transferred to a $20 \mathrm{~mL}$ scintillation vial. Tris(dibenzylideneacetone dipalladium(0) (277.7 mg, $0.303 \mathrm{mmol}, 0.5$ equiv.) was then partially dissolved in tetrahydrofuran $(10 \mathrm{~mL})$ and added to the solution of 1 . The reaction mixture was allowed to stir for 30 minutes. The scintillation vial was then removed from the inert atmosphere glovebox and exposed to air and allowed to stir for 1 hour. The reaction immediately turned a deep purple upon exposure to air. The reaction mixture was then poured onto a silica plug that had been wet packed with ethyl acetate (Note: the product is not indefinitely stable on silica and should be eluted quickly from the plug column). Excess dibenzylideneacetone was eluted with ethyl acetate as evidenced by a yellow band. The product was then eluted with methanol taking care to collect only the purple band. The methanol filtrate was then dried under reduced pressure. The purple residue was dried on the Schlenk line overnight and brought into the glovebox where it was dissolved in dry dichloromethane and redried under reduced pressure to yield the product as a purple powder. Yield: $219.7 \mathrm{mg}(60 \%) .{ }^{1} \mathrm{H}$ NMR $\left(300 \mathrm{MHz}, \mathrm{CD}_{2} \mathrm{Cl}_{2}\right) \delta 7.62-7.54(\mathrm{~m}, \mathrm{ArH}, 2 \mathrm{H}), 7.53-7.48(\mathrm{~m}, \mathrm{ArH}, 4 \mathrm{H}), 7.47-7.37$ (m, $\mathrm{ArH}, 2 \mathrm{H}), 6.10$ (s, central ArH, 2H), 2.46 (dtt, CH, 2H), 2.33 (m, CH, 2H), 1.13 (m, $\mathrm{CH}_{3}$, $24 \mathrm{H}) .{ }^{13} \mathrm{C}\left\{{ }^{1} \mathrm{H}\right\}$ NMR $\left(126 \mathrm{MHz}, \mathrm{CD}_{2} \mathrm{Cl}_{2}\right) \delta 165.45(\mathrm{~s}), 146.28-145.30(\mathrm{~m}), 140.90-139.75$ (m), 132.41 (s), 130.78 (s), 129.82 (s), 128.81 (vt, JPC $=7.9 \mathrm{~Hz}), 127.82$ (d, JPC = $2.5 \mathrm{~Hz}$ ), $102.64(\mathrm{~s}), 28.18-27.56(\mathrm{~m}), 26.85-25.79(\mathrm{~m}), 19.38\left(\mathrm{t}, \mathrm{JPC}_{\mathrm{PC}}=3.7 \mathrm{~Hz}\right), 18.70$ (vt, JPC $=3.6$ $\mathrm{Hz}), 18.34$ (s), 18.05 (s). ${ }^{31} \mathrm{P}\left\{{ }^{1} \mathrm{H}\right\} \mathrm{NMR}\left(121 \mathrm{MHz}, \mathrm{CD}_{2} \mathrm{Cl}_{2}\right) \delta 55.02$ (s). IR ( $\mathrm{CaF}_{2}$ window, THF, $\left.\mathrm{cm}^{-1}\right) v_{\mathrm{C}-\mathrm{O}}$ : 1603. Anal. Calcd. for: $\mathrm{C}_{30} \mathrm{H}_{38} \mathrm{FO}_{4} \mathrm{P}_{2} \mathrm{Pd}(3)(\%): \mathrm{C}, 60.16 ; \mathrm{H}, 6.39$. Anal. Calcd. for: $\mathrm{C}_{31} \mathrm{H}_{40} \mathrm{Cl}_{2} \mathrm{O}_{4} \mathrm{P}_{2} \mathrm{Pd}(3 \cdot \mathbf{D C M})(\%)$ : C, 54.44; H, 5.90. Found: C, 54.90; H, 6.00.

\section{Synthesis of Compound 4-H}

Compound 1-H (300 mg, 0.606 mmol, 1 equiv.) was dissolved in tetrahydrofuran (10 $\mathrm{mL}$ ) and transferred to a Schlenk tube fitted with a screw-in Teflon stopper. (1,5cyclooctadiene)palladium(II) dichloride (173.2 mg, $0.606 \mathrm{mmol}, 1$ equiv.) was then added as a partially dissolved suspension in tetrahydrofuran $(15 \mathrm{~mL})$. The reaction mixture was allowed to stir for 2 hours during which time the reaction became a homogeneous orange solution. (1,5-cyclooctadiene)(2,2'-bipyridine)nickel(0) (195.6 mg, $0.606 \mathrm{mmol}, 1$ equiv.) was then dissolved in tetrahydrofuran $(15 \mathrm{~mL})$ and transferred to the reaction mixture. This resulted in an immediate color change to a heterogeneous brown/orange suspension. After stirring for 15 minutes the reaction mixture was filtered through a Celite pad with washing with additional tetrahydrofuran until washes became less colored. The combined tetrahydrofuran filtrate was dried under reduced pressure to yield a brown residue. This residue was then redissolved in benzene and lyophilized to yield the product as a brown powder. Yield: $305 \mathrm{mg}(84 \%)$. Note: the product is not indefinitely stable in solution and eventually decomposes to a mixture of species. However, it is stable in the solid state. ${ }^{1} \mathrm{H}$ NMR (300 MHz, C6 $\left.\mathrm{D}_{6}\right) \delta 7.41-7.29(\mathrm{~m}, \mathrm{ArH}, 4 \mathrm{H}), 7.12-7.03$ (m, ArH, 4H), 6.94 (s, central ArH, 2H), 4.14 (s, OH, 2H), 1.88 (m, CH, 4H), 1.35 (m, $\left.\mathrm{CH}_{3}, 12 \mathrm{H}\right), 0.95$ (m, $\mathrm{CH}_{3}$, $12 \mathrm{H}) .{ }^{13} \mathrm{C}\left\{{ }^{1} \mathrm{H}\right\}$ NMR $\left(126 \mathrm{MHz}, \mathrm{C}_{6} \mathrm{D}_{6}\right) \delta 145.26(\mathrm{~s}), 144.83$ (vt, JPC $\left.=10.2 \mathrm{~Hz}\right), 135.02$ (vt, JPC $=9.7 \mathrm{~Hz}$ ), 132.01 (s), 129.67 (s), 128.57 (s), 128.19 (s), 124.22 (s), 29.15 (vt, JPC = $6.1 \mathrm{~Hz}$ ), $27.82\left(\mathrm{vt}, \mathrm{JPC}_{\mathrm{PC}}=6.2 \mathrm{~Hz}\right), 22.04\left(\mathrm{vt}, \mathrm{JPC}_{\mathrm{PC}}=8.3 \mathrm{~Hz}\right), 21.82\left(\mathrm{vt}, \mathrm{JPC}_{\mathrm{PC}}=9.1 \mathrm{~Hz}\right), 20.80-20.30(\mathrm{~m})$. ${ }^{31} \mathrm{P}\left\{{ }_{1}^{1} \mathrm{H}\right\}$ NMR (121 MHz, $\left.\mathrm{C}_{6} \mathrm{D}_{6}\right) \delta 33.85$ (s). Anal. Calcd. for: $\mathrm{C}_{30} \mathrm{H}_{40} \mathrm{O}_{2} \mathrm{P}_{2} \mathrm{Pd}(4)(\%): \mathrm{C}$, 59.95; H, 6.71. Found: C, 60.21; H, 6.71 . 


\section{Synthesis of Compound 5-H}

Compound 1-H (100.7 mg, $0.204 \mathrm{mmol}, 1$ equiv.) was dissolved in tetrahydrofuran (8 mL) and added to a $20 \mathrm{~mL}$ scintillation vial containing (1,5-cyclooctadiene)palladium(II) dichloride (58.1 mg, $0.204 \mathrm{mmol}, 1$ equiv.). The reaction mixture was allowed to stir for 2 hours during which time the solution became a homogeneous orange. Silver triflate (52.3 $\mathrm{mg}, 0.204 \mathrm{mmol}, 1$ equiv.) was then added as a tetrahydrofuran solution resulting in an immediate color change to a dark red. The solution was allowed to stir for 30 minutes before filtering the solution through a Celite pad. Tetrahydrofuran washes eluted further product from the Celite pad. The combined tetrahydrofuran filtrate was dried under reduced pressure to yield a red residue. This residue was recrystallized from acetonitrile:diethyl ether to yield the product as dark red crystals. Yield: $56.1 \mathrm{mg}(31.6 \%)$. Note: the product is not indefinitely stable in solution and eventually decomposes to a mixture of species. However, it is stable in the solid state. ${ }^{1} \mathrm{H}$ NMR $\left(300 \mathrm{MHz}, \mathrm{CD}_{3} \mathrm{CN}\right) \delta 8.18$ (broad s, OH, 2H), $7.86(\mathrm{~m}, \mathrm{ArH}, 2 \mathrm{H})$, 7.79 - 7.65 (m, $\mathrm{ArH}, 4 \mathrm{H}), 7.59$ (m', $\mathrm{ArH}, 2 \mathrm{H}), 6.72$ (s, central $\mathrm{ArH}, 2 \mathrm{H}), 3.12(\mathrm{~m}, \mathrm{CH}, 4 \mathrm{H})$, $1.42-1.07\left(\mathrm{~m}, \mathrm{CH}_{3}, 24 \mathrm{H}\right) .{ }^{13} \mathrm{C}\left\{{ }^{1} \mathrm{H}\right\}$ NMR $\left(126 \mathrm{MHz}, \mathrm{CD}_{3} \mathrm{CN}\right) \delta 148.17$ (s), 143.58 (s), 133.80 (s), 133.23 (s), 132.55 (s), 130.76 (s), 130.14 (s), 128.81 (s), 128.28 (s), 27.35 (s), 26.89 $\left(\mathrm{vt}, \mathrm{J}_{\mathrm{PC}}=11.5 \mathrm{~Hz}\right), 19.61(\mathrm{~s}), 19.34(\mathrm{~s}), 18.74(\mathrm{~s}) .{ }^{31} \mathrm{P}\left\{{ }^{1} \mathrm{H}\right\} \mathrm{NMR}\left(121 \mathrm{MHz}, \mathrm{CD}_{3} \mathrm{CN}\right) \delta 34.22$ (s). ${ }^{19} \mathrm{~F}$ NMR $\left(282 \mathrm{MHz}, \mathrm{CD}_{3} \mathrm{CN}\right) \delta-79.40$ (s). Anal. Calcd. for: $\mathrm{C}_{31} \mathrm{H}_{44} \mathrm{~F}_{3} \mathrm{O}_{5} \mathrm{P}_{2} \mathrm{PdS}(5)(\%): \mathrm{C}$, 47.40; H, 4.51. Found: C, 47.62; H, 5.13.

\section{Synthesis of Compound 6-H}

Pyridinium triflate (44.7 $\mathrm{mg}, 0.195 \mathrm{mmol}, 1$ equiv.) was partially dissolved in tetrahydrofuran $(4 \mathrm{~mL})$ in a $20 \mathrm{~mL}$ scintillation vial. Compound $\mathbf{4}-\mathbf{H}(117.2 \mathrm{mg}, 0.195 \mathrm{mmol}$, 1 equiv.) was the added as a tetrahydrofuran solution which resulted in an immediate lightening of the solution from yellow/brown to yellow. After stirring for 16 hours the reaction mixture was dried under reduced pressure to yield the product as a foamy yellow/orange solid in quantitative yield. ${ }^{1} \mathrm{H}$ NMR $\left(300 \mathrm{MHz}, \mathrm{CD}_{3} \mathrm{CN}\right) \delta 7.84-7.75$ (m, $\mathrm{ArH}, 2 \mathrm{H}), 7.71-7.53$ (m, ArH, 6H), 7.42 (broad s, OH, 2H), 6.79 (s, central ArH, 4H), 2.46 (m, CH, 4H), $1.19-1.01\left(\mathrm{~m}, \mathrm{CH}_{3}, 18 \mathrm{H}\right), 0.94\left(\mathrm{~m}, \mathrm{CH}_{3}, 6 \mathrm{H}\right),-16.33$ (t, JPH $=8.5 \mathrm{~Hz}, \mathrm{Pd} H$, $1 \mathrm{H}) .{ }^{13} \mathrm{C}\left\{{ }^{1} \mathrm{H}\right\} \mathrm{NMR}\left(126 \mathrm{MHz}, \mathrm{CD}_{3} \mathrm{CN}\right) \delta 144.58(\mathrm{~s}), 143.99$ (vt, JPC $\left.=7.2 \mathrm{~Hz}\right), 132.28(\mathrm{~s})$, 131.11 (s), 130.12 (s), 128.38 (s), 126.64 (s), 125.97 (vt, JPC = $19.2 \mathrm{~Hz}$ ), 110.30 (s), 28.11 (vt, $\left.\mathrm{J}_{\mathrm{PC}}=13.1 \mathrm{~Hz}\right), 27.30\left(\mathrm{vt}, \mathrm{JPC}_{\mathrm{PC}}=12.5 \mathrm{~Hz}\right), 19.68$ (s), 19.44 (s), 18.60 (s), $18.41(\mathrm{~s}) .{ }^{31} \mathrm{P}\left\{{ }^{1} \mathrm{H}\right\}$ NMR $\left(121 \mathrm{MHz}, \mathrm{CD}_{3} \mathrm{CN}\right) \delta 41.57\left(\mathrm{~d}, \mathrm{~J}_{\mathrm{PH}}=6.7 \mathrm{~Hz}\right) .{ }^{19} \mathrm{~F}$ NMR $\left(282 \mathrm{MHz}, \mathrm{CD}_{3} \mathrm{CN}\right) \delta-79.34$ (s). Anal. Calcd. for: $\mathrm{C}_{31} \mathrm{H}_{41} \mathrm{~F}_{3} \mathrm{O}_{5} \mathrm{P}_{2} \mathrm{PdS}$ (6) (\%): C, 49.57; H, 5.50. Found: C, 49.32; H, 5.36.

\section{Synthesis of Compound 7-H}

Compound 1-H (129.5 mg, $0.262 \mathrm{mmol}, 1$ equiv.) was dissolved in tetrahydrofuran $(8 \mathrm{~mL})$ and then added to a $20 \mathrm{~mL}$ scintillation vial with silver triflate $(67.2 \mathrm{mg}, 0.262 \mathrm{mmol}$, 1 equiv.). The reaction mixture was allowed to stir for 3 hours to yield a pale pink solution before being dried under reduced pressure. The residue was then dissolved in minimal acetonitrile and filtered through a Celite pad, which removed the pinkish color. The product was then recrystallized from an acetonitrile:diethyl ether vapor diffusion to yield white crystalline needles. Yield: $134 \mathrm{mg}(68 \%) .{ }^{1} \mathrm{H}$ NMR $\left(500 \mathrm{MHz}, \mathrm{CD}_{3} \mathrm{CN}\right) \delta 7.81-7.77$ (m, $\operatorname{ArH}, 2 \mathrm{H}), 7.72-7.68\left(\mathrm{t}, \mathrm{J}_{\mathrm{HH}}=7.6 \mathrm{~Hz}, 2 \mathrm{H}\right), 7.63$ (t, $\left.\mathrm{J}_{\mathrm{HH}}=7.7 \mathrm{~Hz}, \mathrm{ArH}, 2 \mathrm{H}\right), 7.58$ (dq, $\mathrm{J}_{\mathrm{HH}}=$ $\left.7.5 \mathrm{~Hz}, \mathrm{~J}_{\mathrm{Hн}}=1.8 \mathrm{~Hz}, \mathrm{ArH}, 2 \mathrm{H}\right), 6.98$ (broad s, OH, 2H), 6.85 (s, central ArH, 2H), 2.52 (m, $\mathrm{CH}, 4 \mathrm{H}), 1.32-1.15\left(\mathrm{~m}, \mathrm{CH}_{3}, 12 \mathrm{H}\right), 1.02\left(\mathrm{~m}, \mathrm{CH}_{3}, 12 \mathrm{H}\right) \cdot{ }^{13} \mathrm{C}\left\{{ }^{1} \mathrm{H}\right\}$ NMR $(126 \mathrm{MHz}$, $\left.\mathrm{CD}_{3} \mathrm{CN}\right) \delta 147.80$ (s), 144.47 (vt, $\mathrm{JPC}_{\mathrm{PC}}=8.7 \mathrm{~Hz}$ ), 132.62 (d), 131.00 (s), 130.30 (s), 128.58 (s), 
$126.91\left(\mathrm{~d}, \mathrm{~J}_{\mathrm{PC}}=5.3 \mathrm{~Hz}\right), 126.88-126.48(\mathrm{~m}), 118.16(\mathrm{~s}), 26.12(\mathrm{dd}), 25.78-25.34(\mathrm{~m}), 20.93$ $\left(\mathrm{vt}, \mathrm{J}_{\mathrm{PC}}=5.9 \mathrm{~Hz}\right), 20.65\left(\mathrm{vt}, \mathrm{J}_{\mathrm{PC}}=5.9 \mathrm{~Hz}\right), 19.50(\mathrm{~m}) .{ }^{31} \mathrm{P}\left\{{ }^{1} \mathrm{H}\right\} \mathrm{NMR}\left(121 \mathrm{MHz}, \mathrm{CD}_{3} \mathrm{CN}\right) \delta$ $28.33\left(\mathrm{~d}, \mathrm{~J}_{\mathrm{PAg}}=559.7 \mathrm{~Hz}\right), 28.33\left(\mathrm{~d}, \mathrm{~J}_{\mathrm{PAg}}=484.9 \mathrm{~Hz}\right) .{ }^{19} \mathrm{~F}$ NMR $\left(282 \mathrm{MHz}, \mathrm{CD}_{3} \mathrm{CN}\right) \delta-79.32$ (s). Anal. Calcd. for: $\mathrm{C}_{31} \mathrm{H}_{40} \mathrm{AgF}_{3} \mathrm{O}_{5} \mathrm{P}_{2} \mathrm{~S}$ (7) (\%): C, 49.54; H, 5.37. Found: C, 49.77; H, 5.15 .

\section{Synthesis of Compound 4-Me}

Compound 1-Me (327.3 mg, $0.626 \mathrm{mmol}, 1$ equiv.) was dissolved in tetrahydrofuran $(10 \mathrm{~mL})$ and transferred to a Schlenk tube fitted with a screw-in Teflon stopper. (1,5cyclooctadiene)palladium(II) dichloride was then added as a partially dissolved suspension in tetrahydrofuran $(15 \mathrm{~mL})$. The reaction mixture was allowed to stir for 2 hours during which time the solution became a homogeneous yellow/orange. (1,5-cyclooctadiene)(2,2'bipyridine)nickel(0) was added as a solution in tetrahydrofuran $(20 \mathrm{~mL})$ which resulted in immediate consumption of the purple nickel complex and the formation of a heterogeneous orange solution. The reaction mixture was stirred for an additional 30 minutes before filtering through a Celite pad. Additional tetrahydrofuran was used to wash product through the Celite pad. The combined tetrahydrofuran filtrate was dried under reduced pressure to yield an orange residue. This residue was redissolved in benzene and lyophilized to yield the product as an orange powder. Yield: $385.8 \mathrm{mg}(98 \%) .{ }^{1} \mathrm{H}$ NMR $\left(300 \mathrm{MHz}, \mathrm{C}_{6} \mathrm{D}_{6}\right) \delta 7.63$ (m, $\mathrm{ArH}, 2 \mathrm{H}), 7.45$ (m, ArH, 12H), 7.22 (m, ArH, 4H), 6.86 (s, central ArH, 2H), 3.49 (s, $\mathrm{OCH}_{3}$, $6 \mathrm{H}), 1.96(\mathrm{~m}, \mathrm{CH}, 4 \mathrm{H}), 1.36\left(\mathrm{~m}, \mathrm{CH}_{3}, 12 \mathrm{H}\right), 1.00\left(\mathrm{~m}, \mathrm{CH}_{3}, 12 \mathrm{H}\right) .{ }^{13} \mathrm{C}\left\{{ }^{1} \mathrm{H}\right\}$ NMR $(126 \mathrm{MHz}$, $\left.\mathrm{C}_{6} \mathrm{D}_{6}\right) \delta 149.30(\mathrm{~s}), 147.29\left(\mathrm{vt}, \mathrm{JPC}_{\mathrm{PC}}=10.0 \mathrm{~Hz}\right), 133.88\left(\mathrm{vt}, \mathrm{J}_{\mathrm{PC}}=10.9 \mathrm{~Hz}\right), 131.25$ (s), 129.95 (s), 126.81 (s), 125.74 (s), 55.09 (s), 28.58 (vt, JPC = 6.2 Hz), 28.40 (t, JPC = 6.3 Hz), 22.18 (vt, $\left.\mathrm{J}_{\mathrm{PC}}=8.6 \mathrm{~Hz}\right), 21.97\left(\mathrm{vt}, \mathrm{J}_{\mathrm{PC}}=8.8 \mathrm{~Hz}\right), 20.82(\mathrm{~s}), 20.52(\mathrm{~s}) .{ }^{31} \mathrm{P}\left\{{ }^{1} \mathrm{H}\right\} \mathrm{NMR}\left(121 \mathrm{MHz}, \mathrm{C}_{6} \mathrm{D}_{6}\right) \delta$ 33.81 (s). Anal. Calcd. for: $\mathrm{C}_{32} \mathrm{H}_{44} \mathrm{O}_{2} \mathrm{P}_{2} \mathrm{Pd}(9)$ (\%): C, 61.10; H, 7.05. Found: C, 60.62; H, 6.81.

\section{Synthesis of Compound 6-Me}

Pyridinium triflate (94.8 $\mathrm{mg}, 0.413 \mathrm{mmol}, 1$ equiv.) was partially dissolved in tetrahydrofuran $(5 \mathrm{~mL})$ in a $20 \mathrm{~mL}$ scintillation vial. Compound 4-Me $(260.3 \mathrm{mg}, 0.413$ mmol, 1 equiv.) was then added as a tetrahydrofuran solution $(10 \mathrm{~mL})$ which resulted in an immediate color change from orange to yellow. After stirring for 16 hours the reaction mixture was dried under reduced pressure to yield the product as a foamy yellow/orange solid in quantitative yield. ${ }^{1} \mathrm{H}$ NMR $\left(300 \mathrm{MHz}, \mathrm{CD}_{3} \mathrm{CN}\right) \delta 7.86-7.75(\mathrm{~m}, \mathrm{ArH}, 2 \mathrm{H}), 7.75-$ 7.51 (m, ArH, 6H), 6.99 (s, central ArH, 2H), 3.73 (s, $\left.\mathrm{OCH}_{3}, 1 \mathrm{H}\right), 2.55-2.42(\mathrm{~m}, \mathrm{CH}, 4 \mathrm{H})$, $1.17-1.01\left(\mathrm{~m}, \mathrm{CH}_{3}, 12 \mathrm{H}\right), 1.00-0.84\left(\mathrm{~m}, \mathrm{CH}_{3}, 12 \mathrm{H}\right),-16.33\left(\mathrm{t}, \mathrm{J}_{\mathrm{PH}}=9.0 \mathrm{~Hz}, \mathrm{Pd} H\right.$, $1 \mathrm{H}) .{ }^{13} \mathrm{C}\left\{{ }^{1} \mathrm{H}\right\} \mathrm{NMR}\left(126 \mathrm{MHz}, \mathrm{CD}_{3} \mathrm{CN}\right) \delta 148.03$ (s), 144.17 (vt, J $\mathrm{pC}=7.0 \mathrm{~Hz}$ ), 132.17 (s), 130.98 (s), 128.39 (s), 127.92 (s), 125.55 (vt, JPC = $19.0 \mathrm{~Hz}$ ), 107.70 (s), 56.49 (d), 27.76 (vt, $\left.\mathrm{J}_{\mathrm{PC}}=13.3 \mathrm{~Hz}\right), 27.18\left(\mathrm{vt}, \mathrm{J}_{\mathrm{PC}}=13.2 \mathrm{~Hz}\right), 19.63(\mathrm{~s}), 19.24(\mathrm{~s}), 18.55(\mathrm{~s}), 18.22(\mathrm{~s}) .{ }^{31} \mathrm{P}\left\{{ }^{1} \mathrm{H}\right\}$ NMR (121 MHz, CD 3 CN) $\delta 41.93$ (s). ${ }^{19} \mathrm{~F}$ NMR (282 MHz, CD 3 CN) $\delta-79.37$ (s). Anal. Calcd. for: $\mathrm{C}_{33} \mathrm{H}_{45} \mathrm{~F}_{3} \mathrm{O}_{5} \mathrm{P}_{2} \mathrm{PdS}$ (10) (\%): C, 50.87; H, 5.82. Found: C, 50.19; H, 5.47.

\section{In Situ Preparation of Compound 8-Me}

4-Me (11.6 mg, $0.0184 \mathrm{mmol}, 1$ equiv) was dissolved $\mathrm{d}_{8}$-THF and transferred to a JYoung tube. The solution was then frozen and the headspace removed under vacuum. Excess $\mathrm{O}_{2}(1 \mathrm{~atm})$ was then added and the solution was carefully mixed to avoid warming the solution. Immediate and quantitative conversion to 8-Me was observed by NMR at -78 ${ }^{\circ} \mathrm{C}$. Note: The compound is stable at low temperatures with no decomposition detected by NMR for hours at $-40{ }^{\circ} \mathrm{C}$ or two weeks at $-78{ }^{\circ} \mathrm{C}$. However, around $0{ }^{\circ} \mathrm{C}$ decomposition to a mixture of species in observed. Solutions can be degassed to remove excess $\mathrm{O}_{2}$ by placing 
solutions under dynamic vacuum at $-78{ }^{\circ} \mathrm{C}$ if prepared in a Schlenk tube or by multiple freeze-pump-thaw cycles if care is taken not to warm the solution above $-40{ }^{\circ} \mathrm{C}$. All NMR characterization was obtained at $-40{ }^{\circ} \mathrm{C} .{ }^{1} \mathrm{H}$ NMR $\left(500 \mathrm{MHz}, \mathrm{d}_{8}-\mathrm{THF}\right) \delta 7.62\left(\mathrm{dd}, \mathrm{J}_{\mathrm{HH}}=7.8\right.$ $\left.\mathrm{Hz}, \mathrm{J}_{\mathrm{HH}}=5.1 \mathrm{~Hz}, \operatorname{ArH}, 1 \mathrm{H}\right), 7.54\left(\mathrm{dd}, \mathrm{J}_{\mathrm{HH}}=7.8, \mathrm{~J}_{\mathrm{HH}}=5.1 \mathrm{~Hz}, \operatorname{ArH}, 1 \mathrm{H}\right), 7.52-7.46(\mathrm{~m}$, $\mathrm{ArH}, 4 \mathrm{H}), 7.44-7.38$ (m, ArH, 2H), 6.87 (s, central aryl ArH, 1H), 6.66 (s, central aryl ArH, $1 \mathrm{H}), 3.56\left(\mathrm{~s}, \mathrm{OCH}_{3}, 3 \mathrm{H}\right), 3.47\left(\mathrm{~s}, \mathrm{OCH}_{3}, 3 \mathrm{H}\right), 2.67(\mathrm{~m}, \mathrm{CH}, 1 \mathrm{H}), 2.59(\mathrm{~m}, \mathrm{CH}, 1 \mathrm{H}), 1.98(\mathrm{~m}$, $\mathrm{CH}, 2 \mathrm{H}), 1.37\left(\mathrm{~m}, \mathrm{CH}_{3}, 12 \mathrm{H}\right), 1.13-1.04\left(\mathrm{~m}, \mathrm{CH}_{3}, 6 \mathrm{H}\right), 0.85\left(\mathrm{~m}, \mathrm{CH}_{3}, 6 \mathrm{H}\right) .{ }^{13} \mathrm{C}\left\{{ }^{1} \mathrm{H}\right\} \mathrm{NMR}$ $\left(126 \mathrm{MHz}, \mathrm{d}_{8}\right.$-THF) $\delta 151.03$ (s), $149.81(\mathrm{~s}), 146.60\left(\mathrm{~d}, \mathrm{~J}_{\mathrm{PC}}=16.0 \mathrm{~Hz}\right), 145.46\left(\mathrm{~d}, \mathrm{~J}_{\mathrm{PC}}=16.1\right.$ $\mathrm{Hz}$ ), 133.84 (s), 133.06 (s), 132.99 (s), 132.77 (s), 130.28 (s), 130.09 (s), 129.66 (d, JPC = 6.1 $\mathrm{Hz}), 129.52\left(\mathrm{~d}, \mathrm{~J}_{\mathrm{PC}}=5.8 \mathrm{~Hz}\right), 129.14(\mathrm{~s}), 128.95\left(\mathrm{~d}, \mathrm{~J}_{\mathrm{PC}}=7.2 \mathrm{~Hz}\right), 128.78(\mathrm{~s}), 128.61\left(\mathrm{~d}, \mathrm{~J}_{\mathrm{PC}}=\right.$ 6.6 Hz), 128.21 (s), 127.97 (s), 127.87 (s), 127.67 (s), 127.48 (s), 126.06 (s), 125.53 (s), 113.70 $\left(\mathrm{d}, \mathrm{J}_{\mathrm{PC}}=13.2 \mathrm{~Hz}\right), 54.43(\mathrm{~s}), 28.36\left(\mathrm{~d}, \mathrm{~J}_{\mathrm{PC}}=14.2 \mathrm{~Hz}\right), 27.26\left(\mathrm{~d}, \mathrm{~J}_{\mathrm{PC}}=11.5 \mathrm{~Hz}\right), 25.48\left(\mathrm{~d}, \mathrm{~J}_{\mathrm{PC}}=\right.$ $20.2 \mathrm{~Hz}), 22.70\left(\mathrm{~d}, \mathrm{~J}_{\mathrm{PC}}=18.0 \mathrm{~Hz}\right.$ ), $22.37\left(\mathrm{~d}, \mathrm{~J}_{\mathrm{PC}}=15.1 \mathrm{~Hz}\right), 20.04(\mathrm{~s}), 19.53$ (d, JPC = 13.1 $\mathrm{Hz}), 19.33\left(\mathrm{~d}, \mathrm{JPC}_{\mathrm{PC}}=7.1 \mathrm{~Hz}\right), 18.86\left(\mathrm{~d}, \mathrm{JPC}_{\mathrm{PC}}=5.6 \mathrm{~Hz}\right), 17.26\left(\mathrm{~d}, \mathrm{~J}_{\mathrm{PC}}=4.5 \mathrm{~Hz}\right), 16.32\left(\mathrm{~d}, \mathrm{~J}_{\mathrm{PC}}=\right.$ $6.0 \mathrm{~Hz}) .{ }^{31} \mathrm{P}\left\{{ }^{1} \mathrm{H}\right\} \mathrm{NMR}\left(202 \mathrm{MHz}, \mathrm{d}_{8}-\mathrm{THF}\right) \delta 34.04\left(\mathrm{~d},{ }^{2} \mathrm{Jpp}_{\mathrm{Pp}}=23.8 \mathrm{~Hz}\right), 29.87\left(\mathrm{~d},{ }^{2} \mathrm{~J}_{\mathrm{Pp}}=24.0\right.$ $\mathrm{Hz})$.

\section{Synthesis of Compound (3) $\mathrm{H}^{+}$}

6-H (13.9 $\mathrm{mg}, 0.019 \mathrm{mmol}, 1$ equiv) was dissolved in $\mathrm{CD}_{3} \mathrm{CN}$ and transferred to a JYoung tube. The solution was then frozen and the headspace removed under vacuum. Excess $\mathrm{O}_{2}(1 \mathrm{~atm})$ was then added and the sample was allowed to thaw to room temperature with inversion to ensure adequate mixing. A gradual color change from yellow/orange to a pink/red solution was observed over $6^{+}$hours. NMR revealed quantitative conversion to a new species, $(3) \mathbf{H}^{+}$, that could be isolated as a red powder following removal of volatiles. ${ }^{1} \mathrm{H}$ NMR (300 MHz, CD $\left.{ }_{3} \mathrm{CN}\right) \delta 7.76-7.51(\mathrm{~m}, 8 \mathrm{H}), 6.39(\mathrm{~s}, 2 \mathrm{H}), 2.63(\mathrm{~m}, 2 \mathrm{H}), 2.54(\mathrm{~m}, 2 \mathrm{H})$, $1.24-0.96(\mathrm{~m}, 24 \mathrm{H}) .{ }^{13} \mathrm{C}$ NMR $\left(126 \mathrm{MHz}, \mathrm{CD}_{3} \mathrm{CN}\right) \delta 142.35\left(\mathrm{~d}, \mathrm{~J}_{\mathrm{PC}}=13.7 \mathrm{~Hz}\right), 139.70(\mathrm{~d}$, $\mathrm{J}_{\mathrm{PC}}=37.2 \mathrm{~Hz}$ ), $133.34(\mathrm{~s}), 132.23(\mathrm{~s}), 131.57(\mathrm{~s}), 129.09\left(\mathrm{~d}, \mathrm{~J}_{\mathrm{PC}}=13.6 \mathrm{~Hz}\right), 127.38(\mathrm{~s}), 105.94$ (s), $28.10\left(\mathrm{~d}, \mathrm{~J}_{\mathrm{PC}}=19.1 \mathrm{~Hz}\right), 26.35\left(\mathrm{~d}, \mathrm{~J}_{\mathrm{PC}}=26.1 \mathrm{~Hz}\right), 18.73$ (s), 18.07 (s), 17.83 (s), 17.23 (s).

${ }^{31} \mathrm{P}$ NMR $\left(121 \mathrm{MHz}, \mathrm{CD}_{3} \mathrm{CN}\right) \delta 64.39 .{ }^{19} \mathrm{~F}$ NMR $\left(282 \mathrm{MHz}, \mathrm{CD}_{3} \mathrm{CN}\right) \delta-79.34$.

\section{Nuclear Magnetic Resonance Spectra}

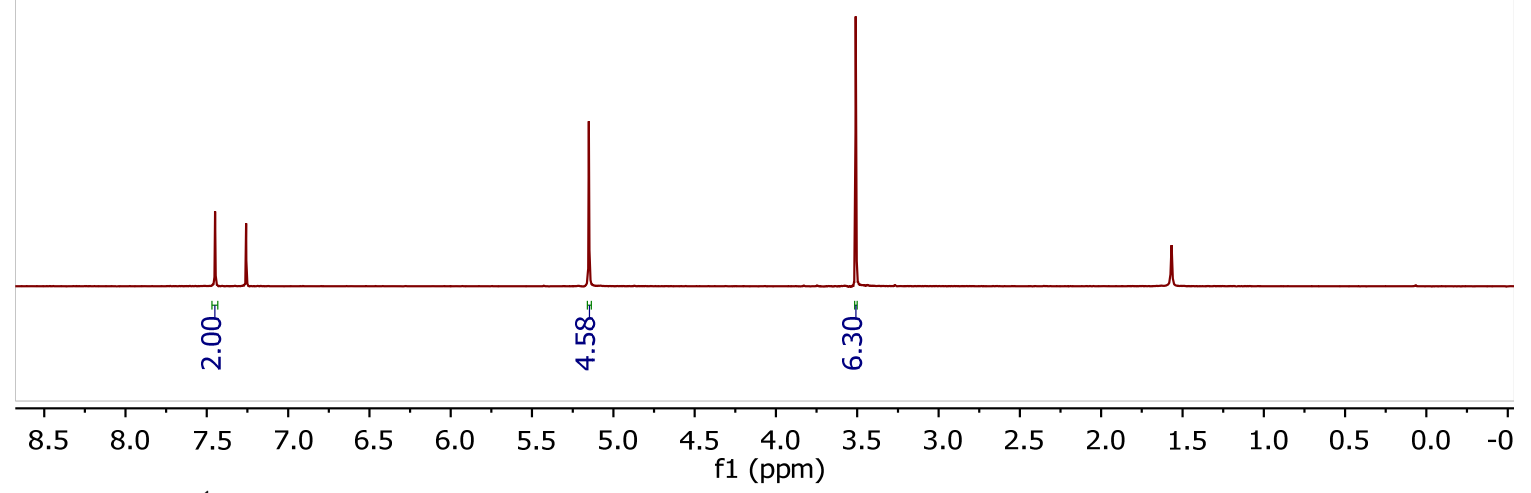

Figure S1. ${ }^{1} \mathrm{H}-\mathrm{NMR}\left(500 \mathrm{MHz}, \mathrm{CDCl}_{3}\right)$ spectrum of $\mathbf{A}$. 


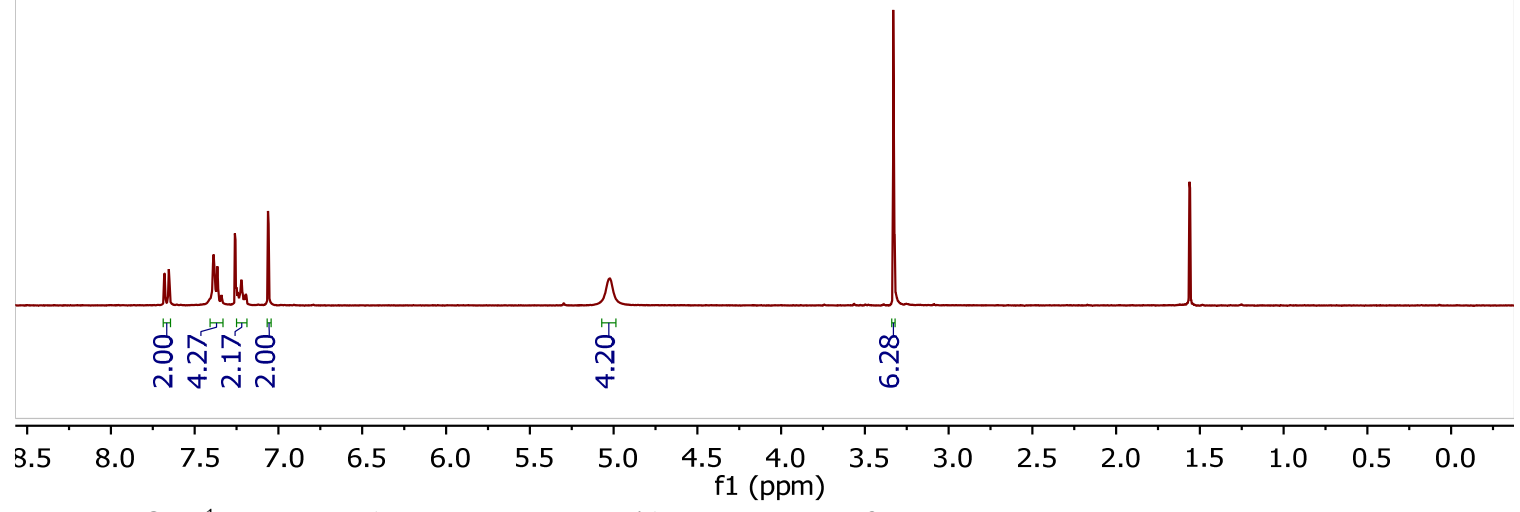

Figure S2. ${ }^{1} \mathrm{H}-\mathrm{NMR}\left(500 \mathrm{MHz}, \mathrm{CDCl}_{3}\right)$ spectrum of $\mathbf{B}$.

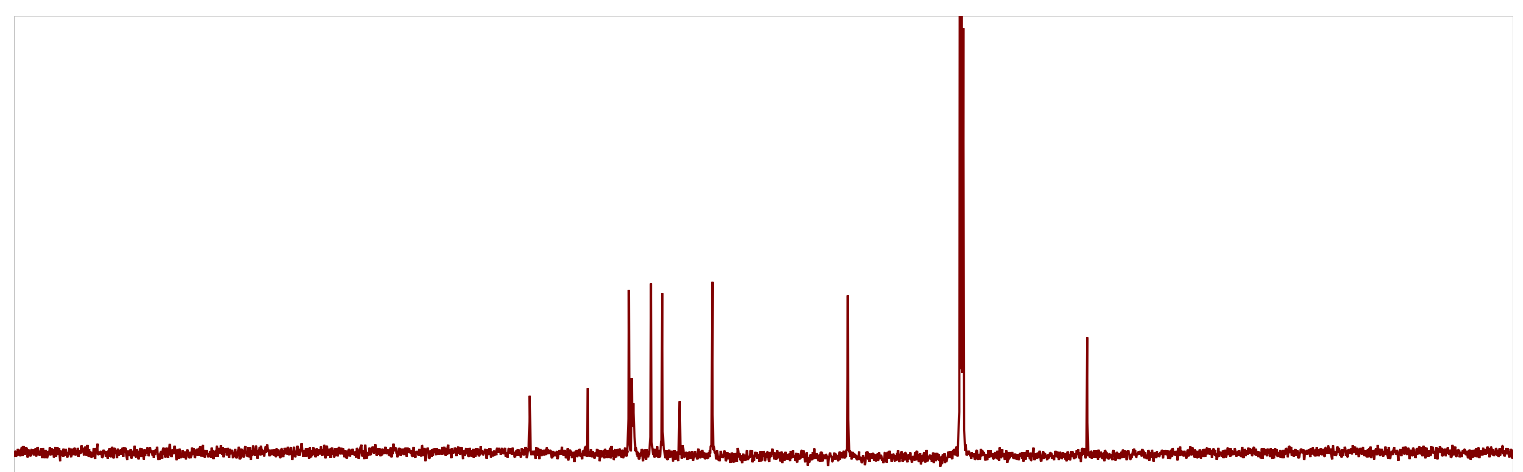

$\begin{array}{llllllllllllllllllllllllllllll}230 & 220 & 210 & 200 & 190 & 180 & 170 & 160 & 150 & 140 & 130 & 120 & 110 & 100 & 90 & 80 & 70 & 60 & 50 & 40 & 30 & 20 & 10 & 0 & -10\end{array}$ f1 (ppm)

Figure S3. ${ }^{13} \mathrm{C}-\mathrm{NMR}\left(126 \mathrm{MHz} \mathrm{CDCl}_{3}\right)$ spectrum of $\mathbf{B}$.

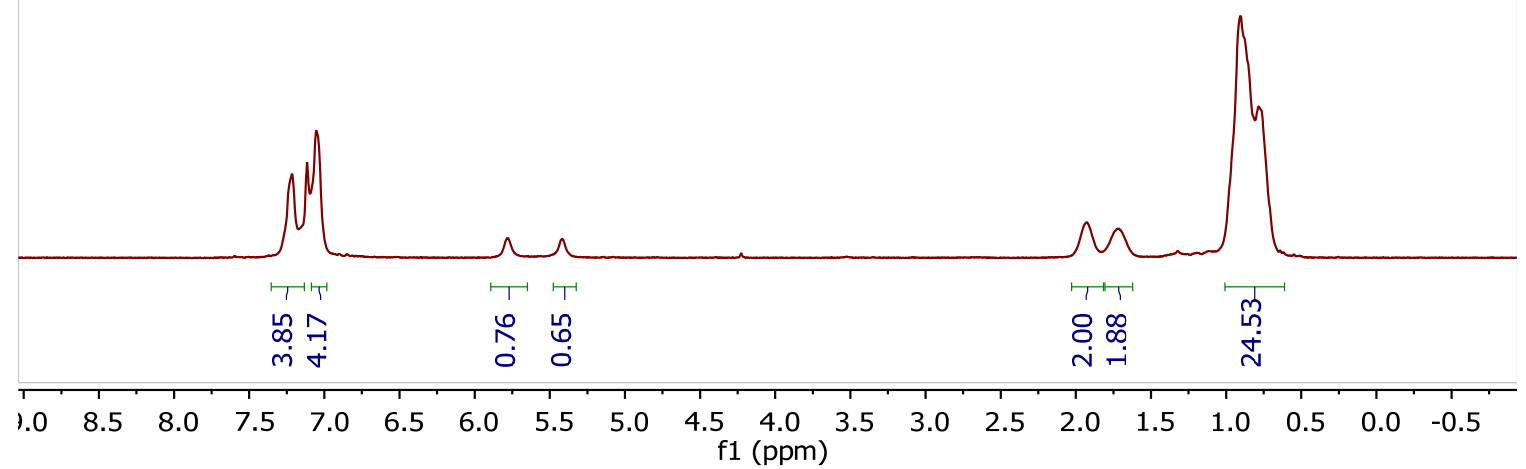

Figure S4. ${ }^{1} \mathrm{H}-\mathrm{NMR}\left(300 \mathrm{MHz}, \mathrm{C}_{6} \mathrm{D}_{6}\right)$ spectrum of $\mathbf{1 - H}$. 


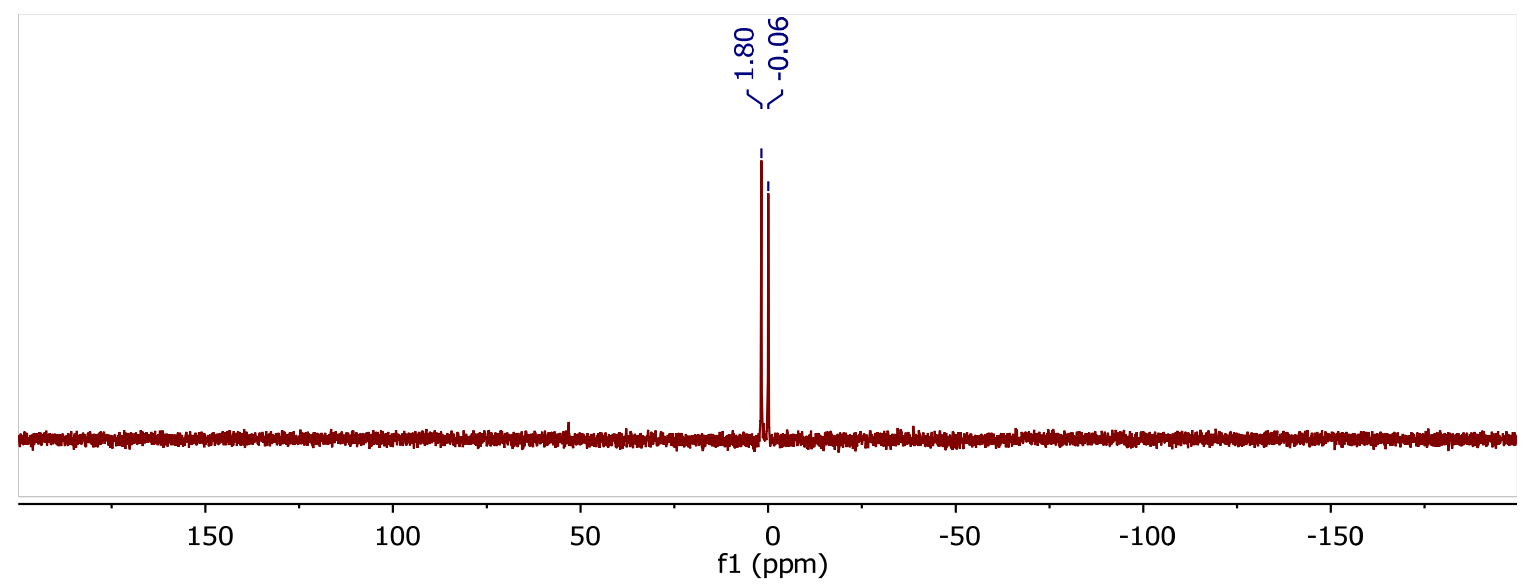

Figure S5. ${ }^{31} \mathrm{P}-\mathrm{NMR}\left(121 \mathrm{MHz}, \mathrm{C}_{6} \mathrm{D}_{6}\right)$ spectrum of $\mathbf{1 - H}$.

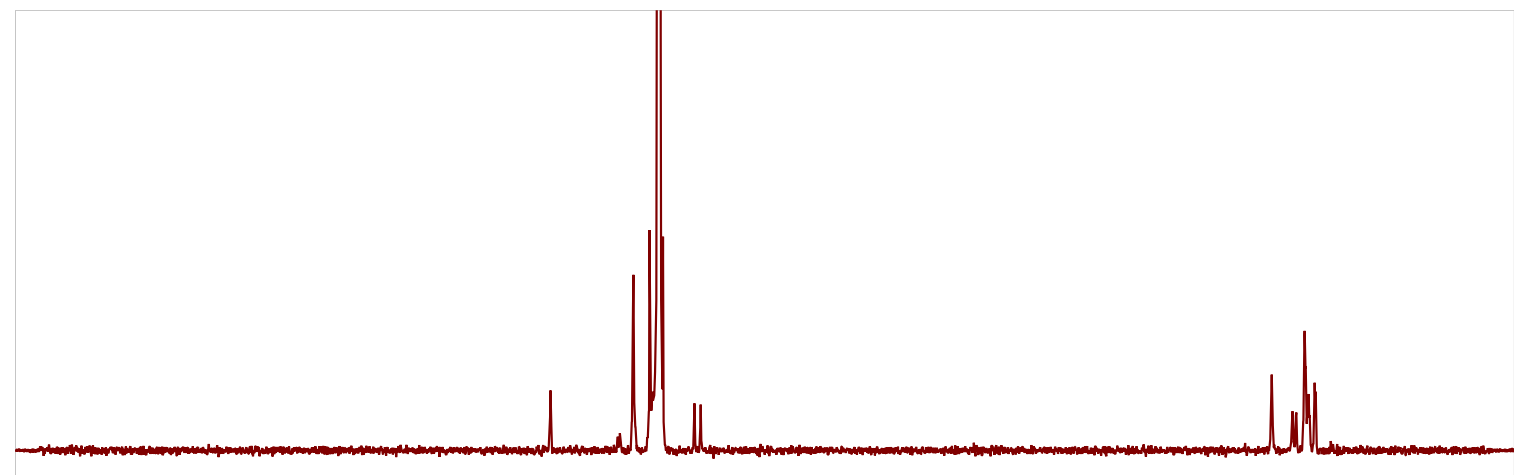

$230220210200190180170160150140 \quad 1301201110100 \begin{array}{lllllllllllll}100 & 80 & 70 & 60 & 50 & 40 & 30 & 20 & 10 & 0 & -10\end{array}$ f1 (ppm)

Figure S6. ${ }^{13} \mathrm{C}-\mathrm{NMR}\left(126 \mathrm{MHz}, \mathrm{C}_{6} \mathrm{D}_{6}\right)$ spectrum of $\mathbf{1 - H}$.

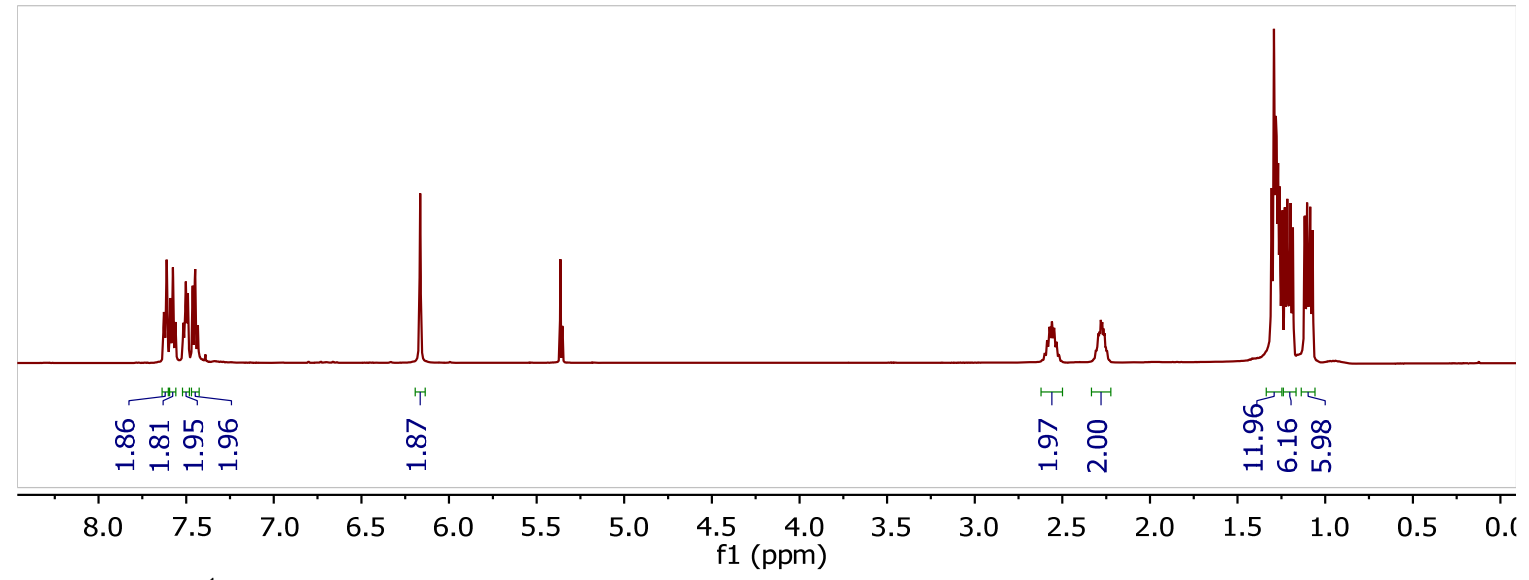

Figure S7. ${ }^{1} \mathrm{H}-\mathrm{NMR}\left(500 \mathrm{MHz}, \mathrm{CD}_{2} \mathrm{Cl}_{2}\right)$ spectrum of 2. 


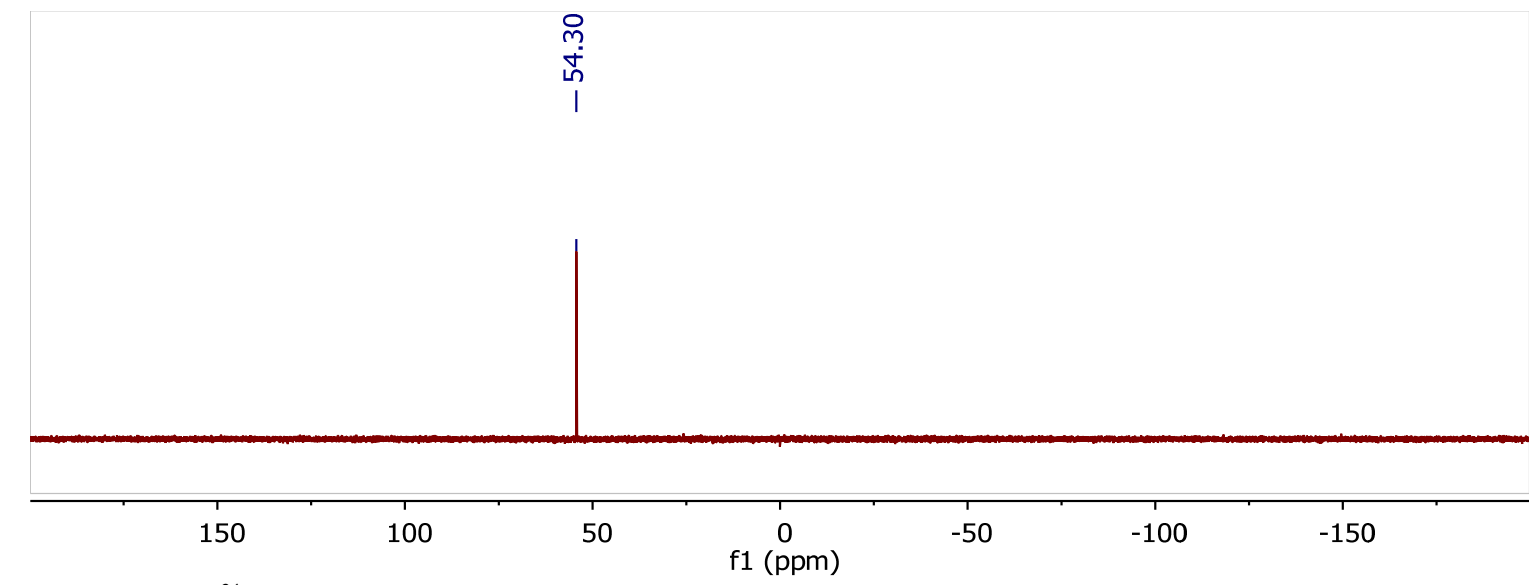

Figure S8. ${ }^{31} \mathrm{P}-\mathrm{NMR}\left(121 \mathrm{MHz}, \mathrm{CD}_{2} \mathrm{Cl}_{2}\right)$ spectrum of 2 .

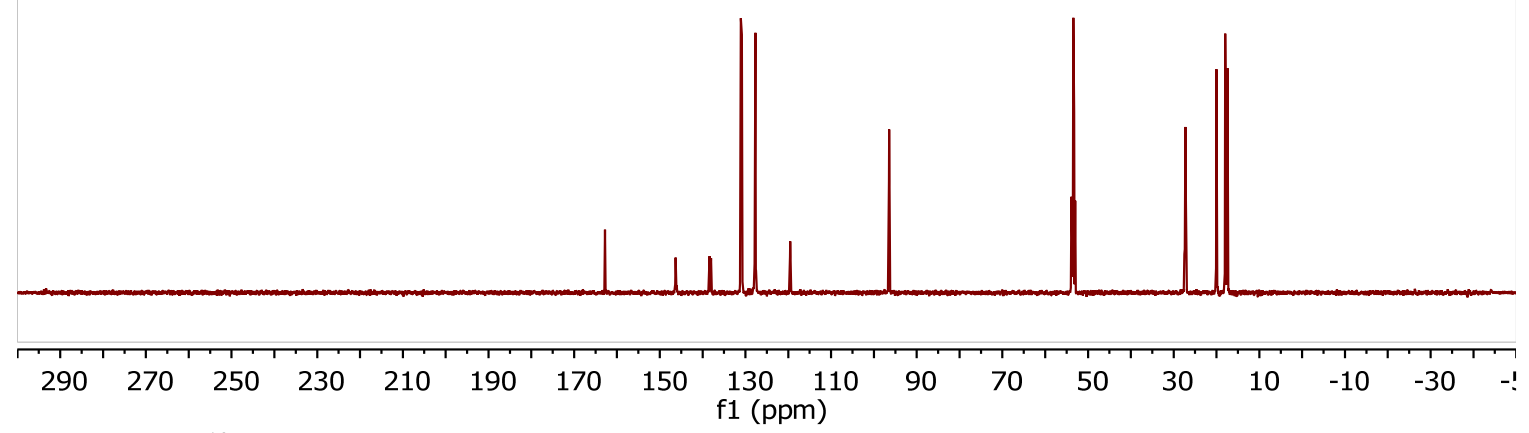

Figure S9. ${ }^{13} \mathrm{C}-\mathrm{NMR}\left(126 \mathrm{MHz}, \mathrm{CD}_{2} \mathrm{Cl}_{2}\right)$ spectrum of 2 .

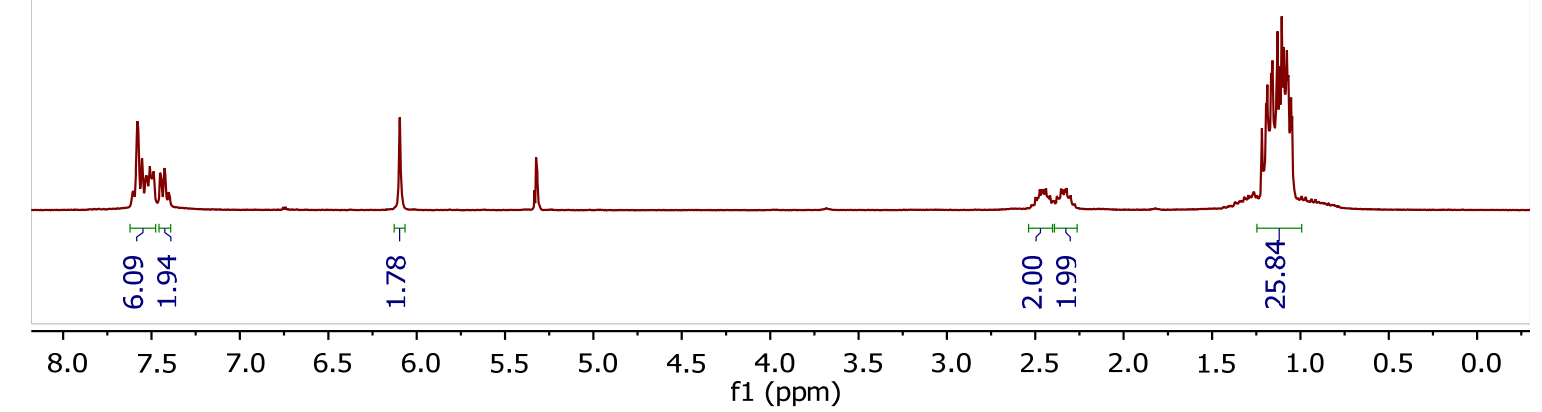

Figure S10. ${ }^{1} \mathrm{H}-\mathrm{NMR}\left(500 \mathrm{MHz}, \mathrm{CD}_{2} \mathrm{Cl}_{2}\right)$ spectrum of 3 . 


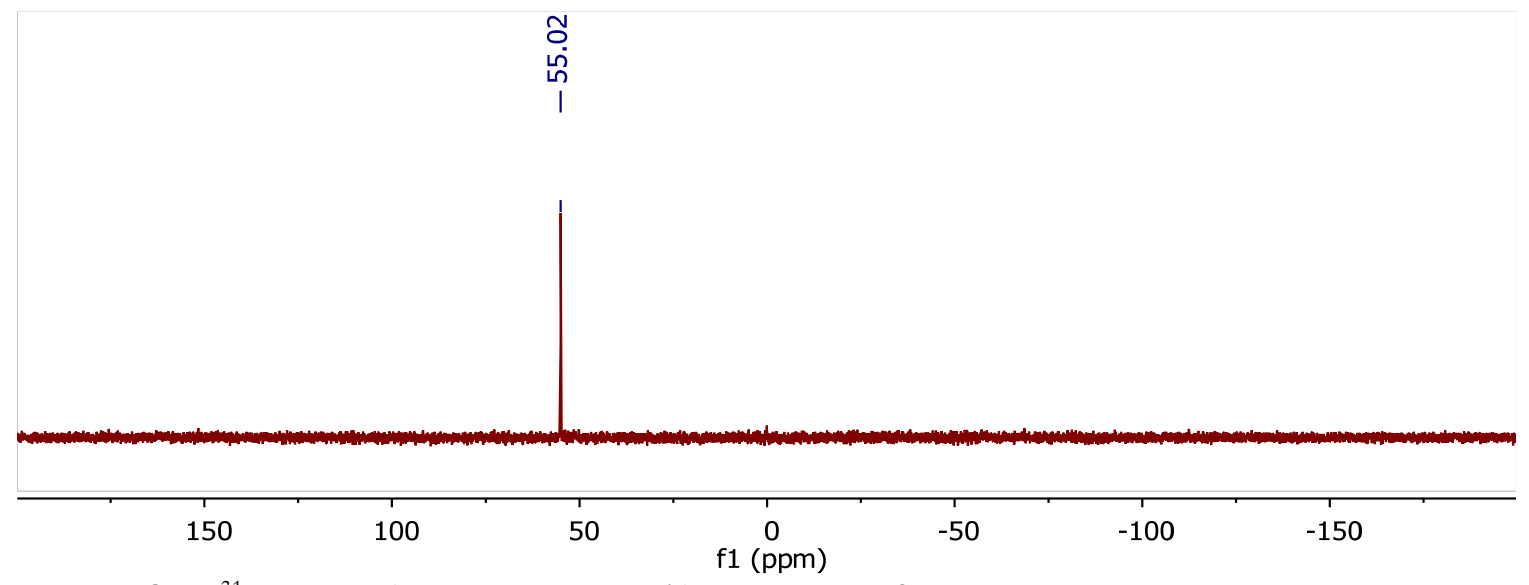

Figure S11. ${ }^{31} \mathrm{P}-\mathrm{NMR}\left(121 \mathrm{MHz}, \mathrm{CD}_{2} \mathrm{Cl}_{2}\right)$ spectrum of $\mathbf{3}$.

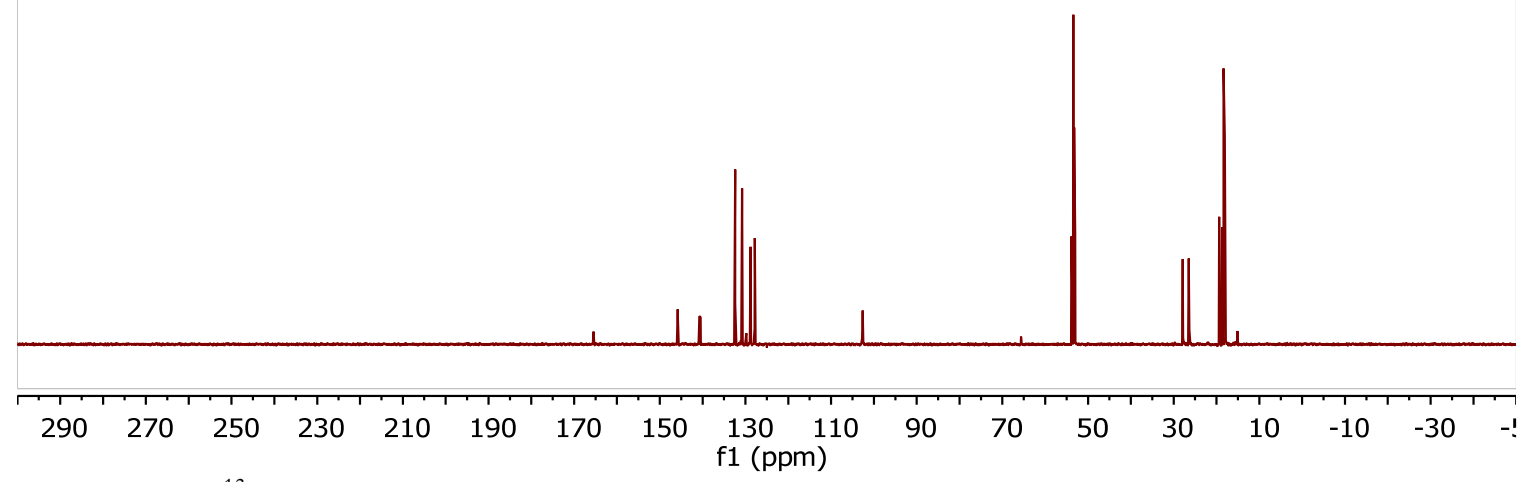

Figure S12. ${ }^{13} \mathrm{C}-\mathrm{NMR}\left(126 \mathrm{MHz}, \mathrm{CD}_{2} \mathrm{Cl}_{2}\right)$ spectrum of $\mathbf{3}$.

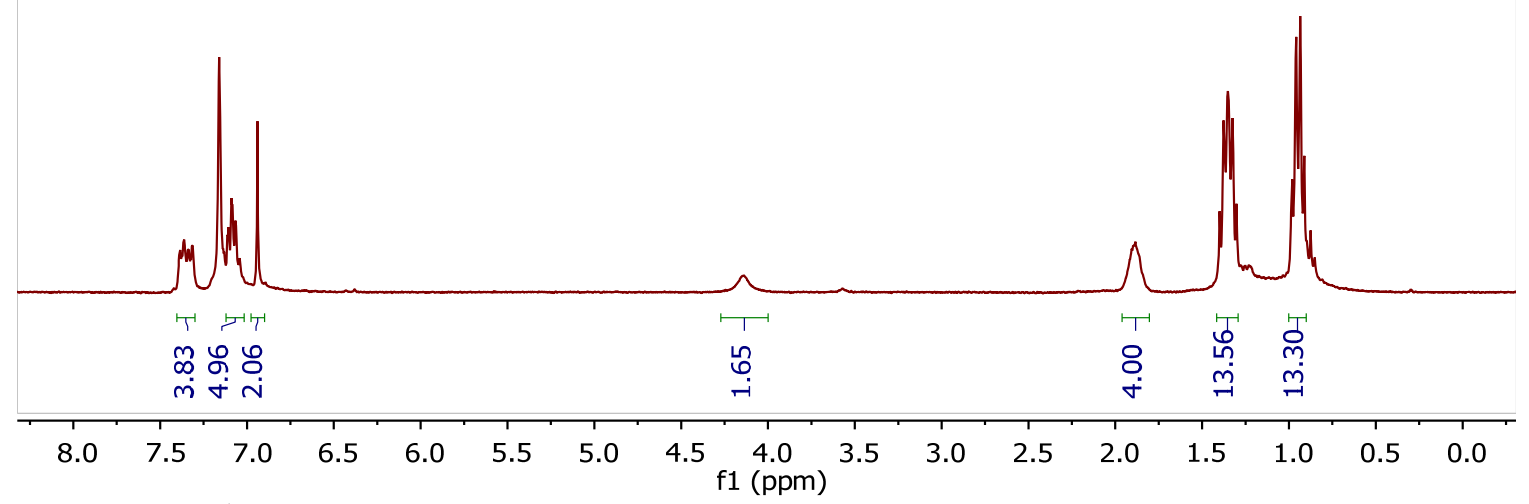

Figure S13. ${ }^{1} \mathrm{H}-\mathrm{NMR}\left(500 \mathrm{MHz}, \mathrm{C}_{6} \mathrm{D}_{6}\right)$ spectrum of $\mathbf{4 - H}$. 


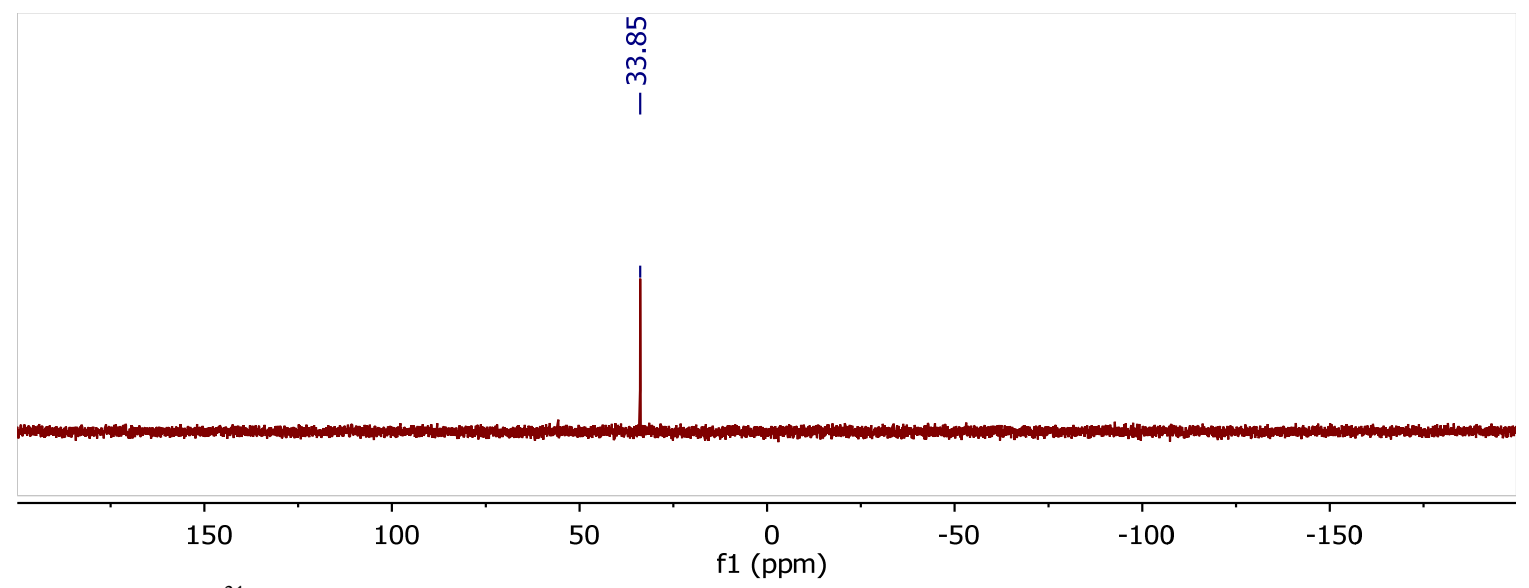

Figure S14. ${ }^{31} \mathrm{P}-\mathrm{NMR}\left(121 \mathrm{MHz}, \mathrm{C}_{6} \mathrm{D}_{6}\right)$ spectrum of $\mathbf{4 - H}$.

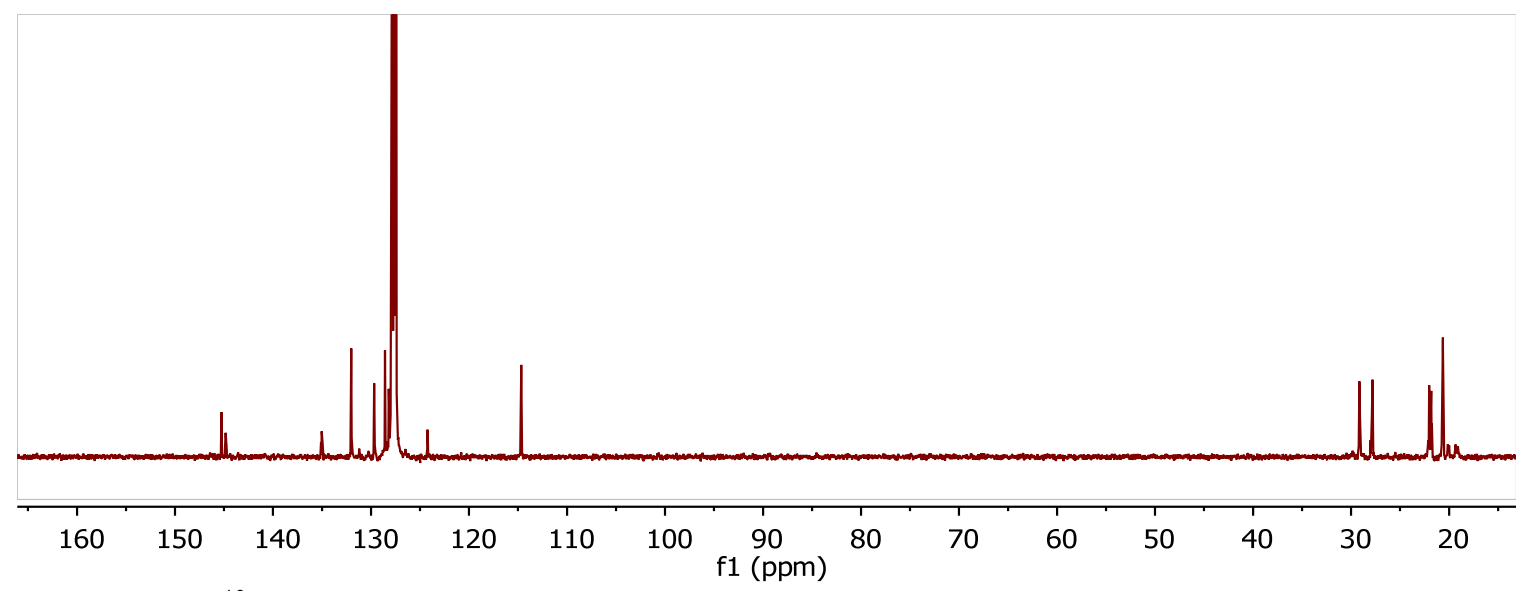

Figure S15. ${ }^{13} \mathrm{C}-\mathrm{NMR}\left(126 \mathrm{MHz}, \mathrm{C}_{6} \mathrm{D}_{6}\right)$ spectrum of $\mathbf{4 - H}$.

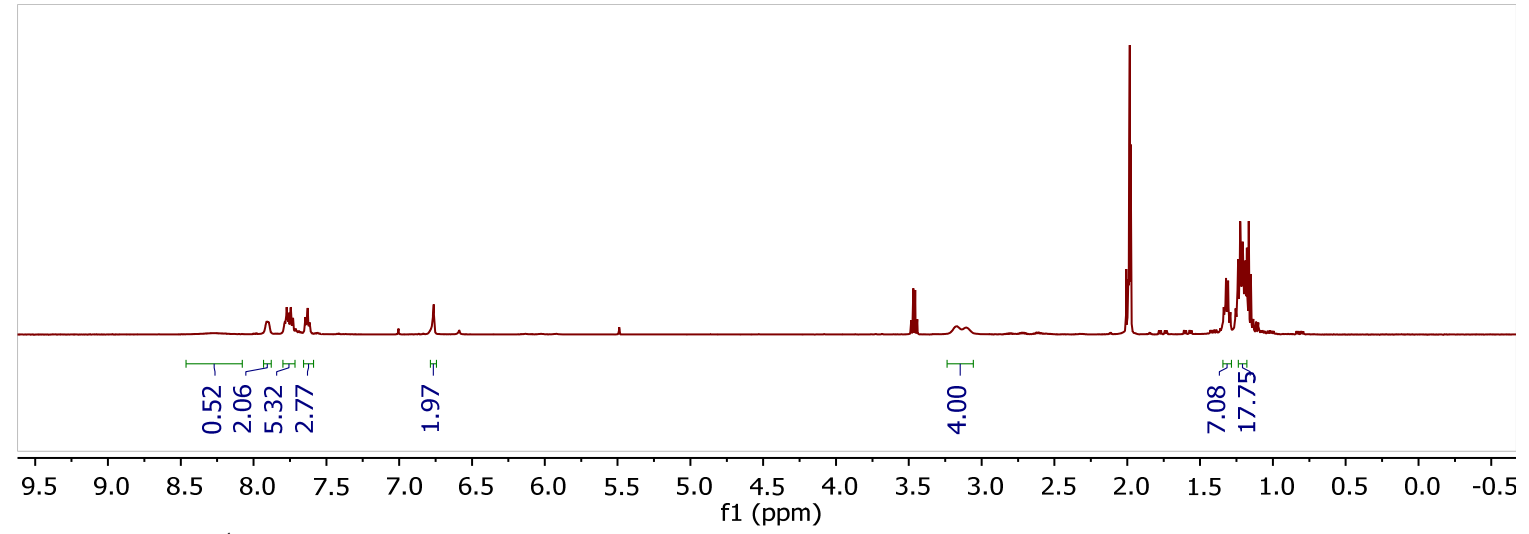

Figure S16. ${ }^{1} \mathrm{H}-\mathrm{NMR}\left(500 \mathrm{MHz}, \mathrm{CD}_{3} \mathrm{CN}\right)$ spectrum of $\mathbf{5}-\mathbf{H}$. 


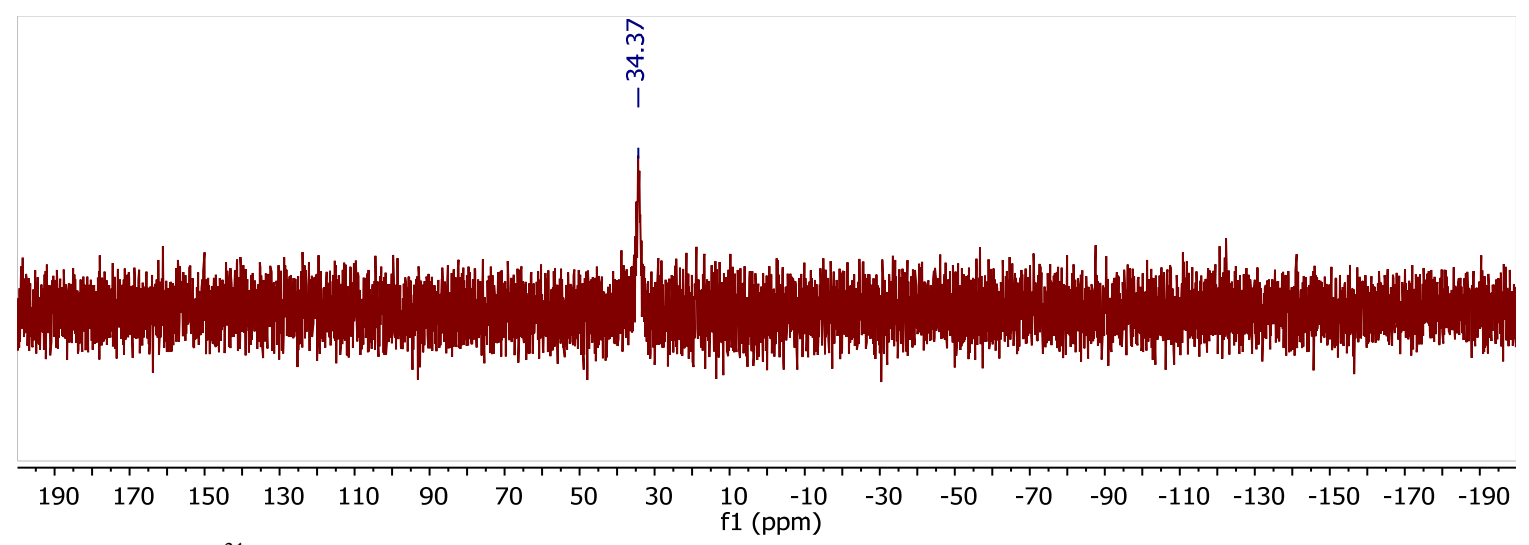

Figure S17. ${ }^{31} \mathrm{P}-\mathrm{NMR}\left(121 \mathrm{MHz}, \mathrm{CD}_{3} \mathrm{CN}\right)$ spectrum of $\mathbf{5 - H}$.

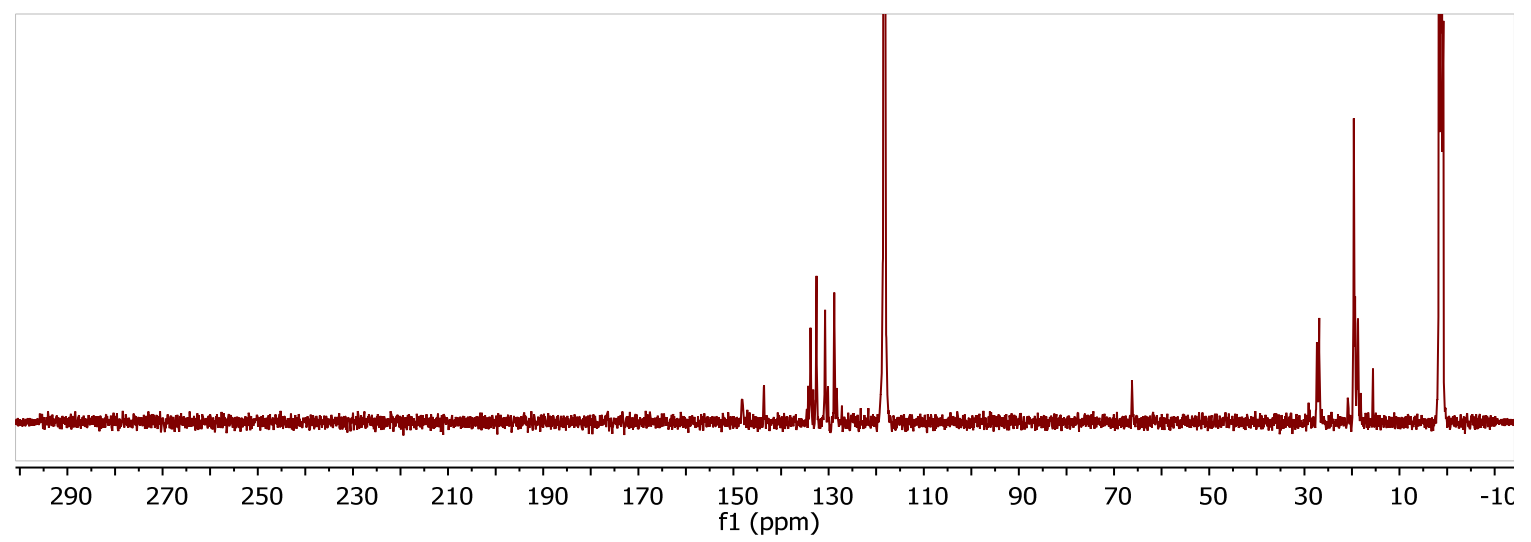

Figure S18. ${ }^{13} \mathrm{C}-\mathrm{NMR}\left(126 \mathrm{MHz}, \mathrm{CD}_{3} \mathrm{CN}\right)$ spectrum of $\mathbf{5 - H}$.

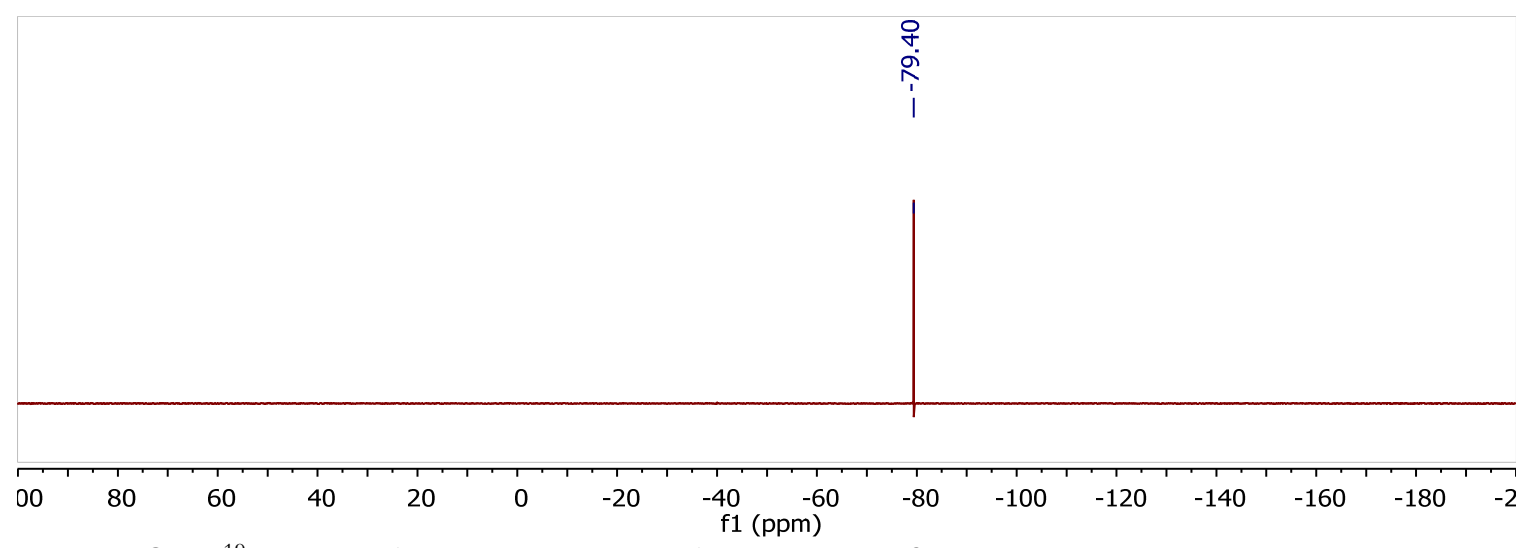

Figure S19. ${ }^{19} \mathrm{~F}-\mathrm{NMR}\left(282 \mathrm{MHz}, \mathrm{CD}_{3} \mathrm{CN}\right)$ spectrum of $\mathbf{5 - H}$. 


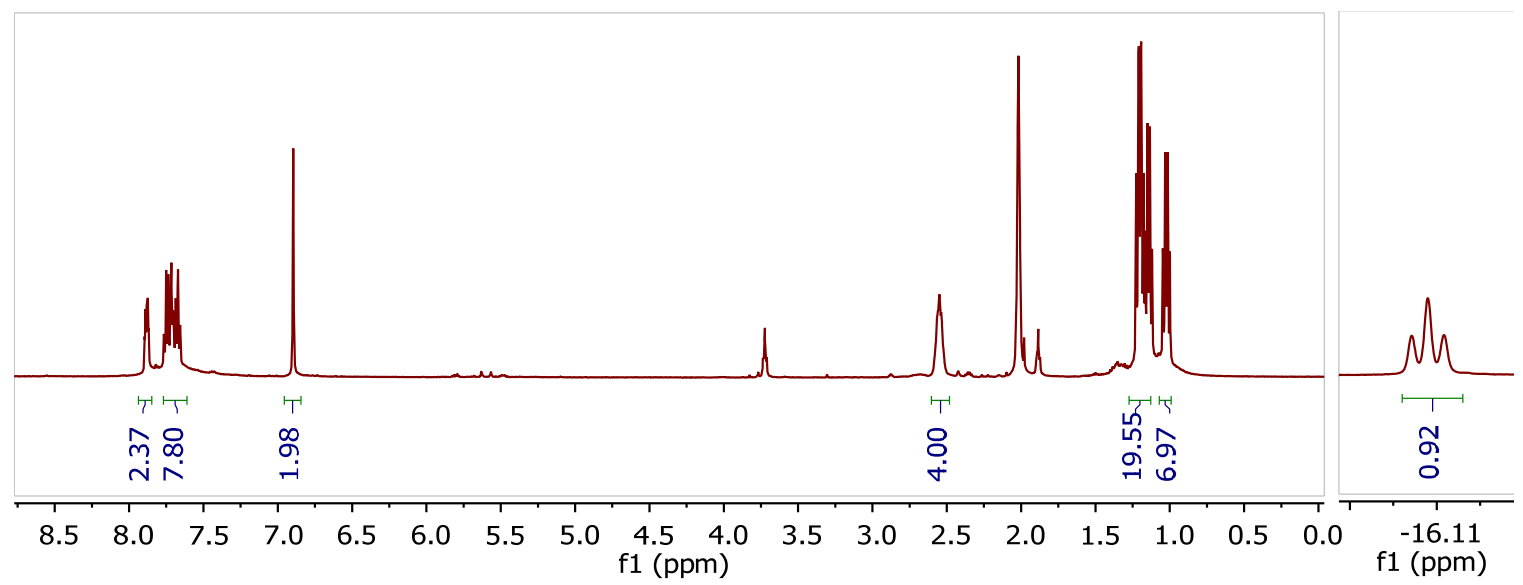

Figure S20. ${ }^{1} \mathrm{H}-\mathrm{NMR}\left(500 \mathrm{MHz}, \mathrm{CD}_{3} \mathrm{CN}\right)$ spectrum of $\mathbf{6 - H}$.

察芒

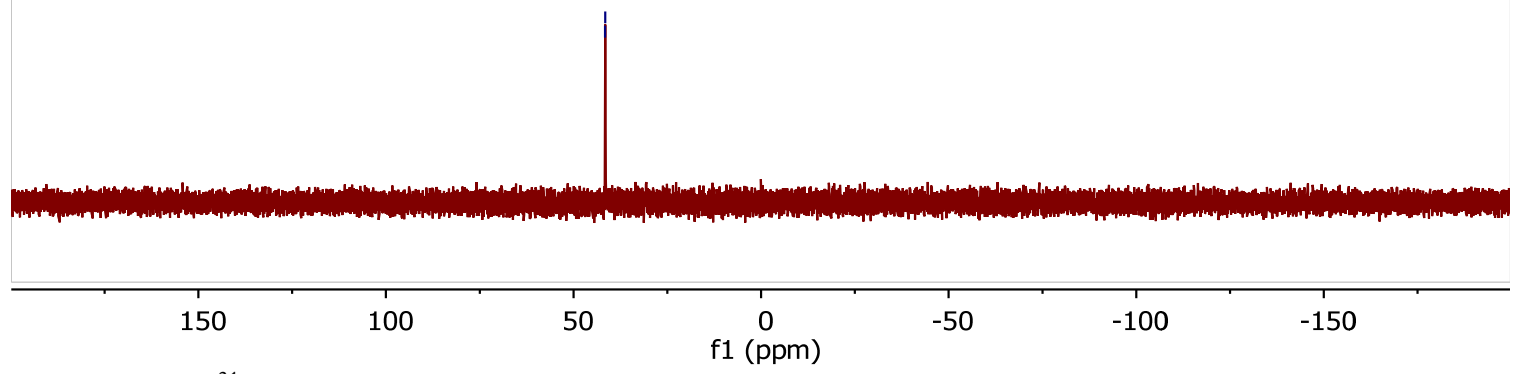

Figure S21. ${ }^{31} \mathrm{P}-\mathrm{NMR}\left(121 \mathrm{MHz}, \mathrm{CD}_{3} \mathrm{CN}\right)$ spectrum of $\mathbf{6}-\mathbf{H}$.

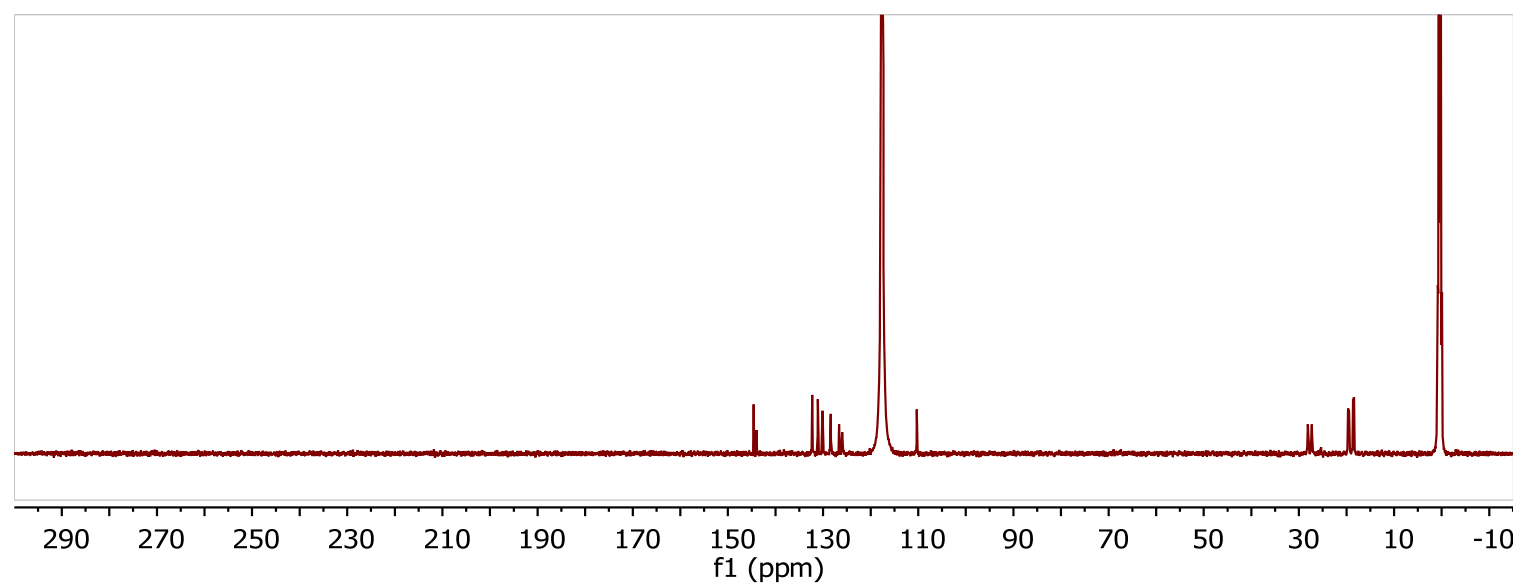

Figure S22. ${ }^{13} \mathrm{C}-\mathrm{NMR}\left(126 \mathrm{MHz}, \mathrm{CD}_{3} \mathrm{CN}\right)$ spectrum of $\mathbf{6}-\mathbf{H}$. 


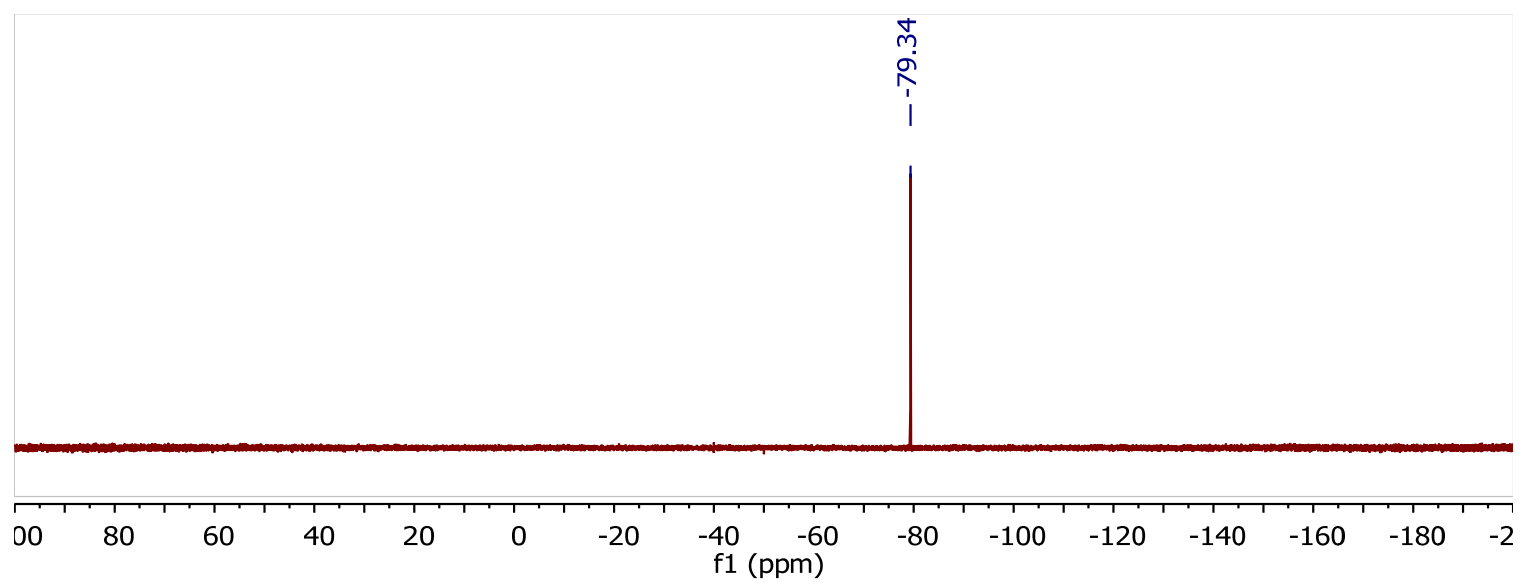

Figure S23. ${ }^{19} \mathrm{~F}-\mathrm{NMR}\left(282 \mathrm{MHz}, \mathrm{CD}_{3} \mathrm{CN}\right)$ spectrum of $\mathbf{6 - H}$.

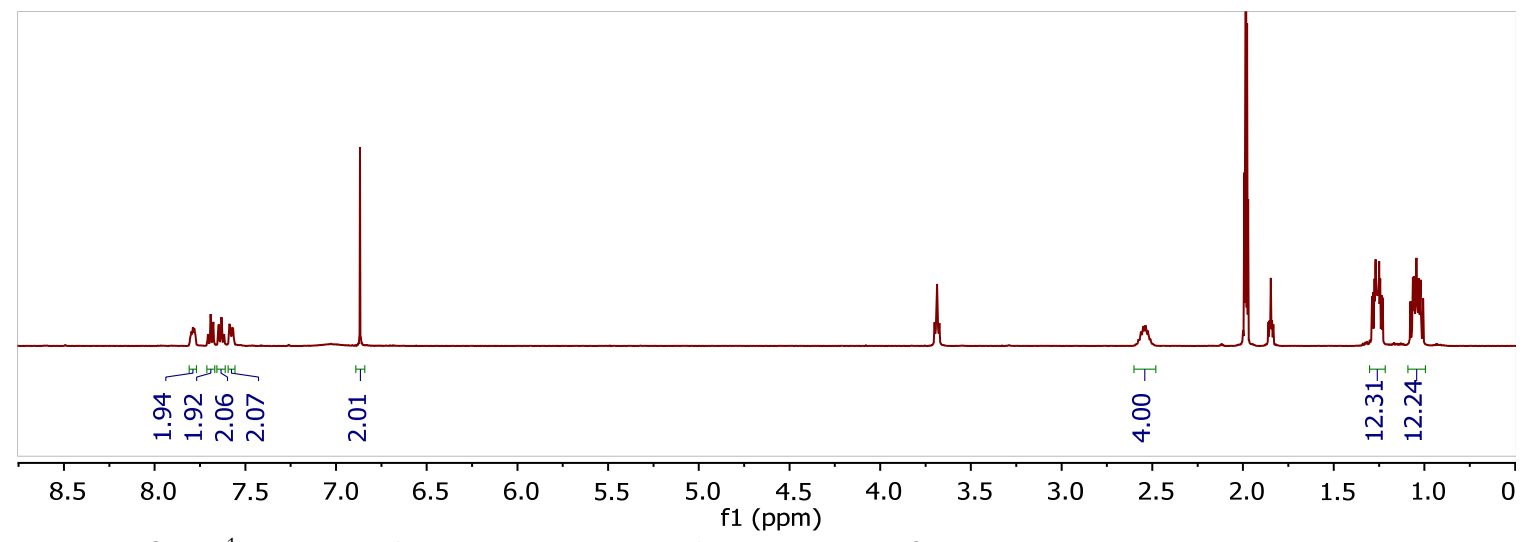

Figure S24. ${ }^{1} \mathrm{H}-\mathrm{NMR}\left(500 \mathrm{MHz}, \mathrm{CD}_{3} \mathrm{CN}\right)$ spectrum of $\mathbf{7 - H}$.

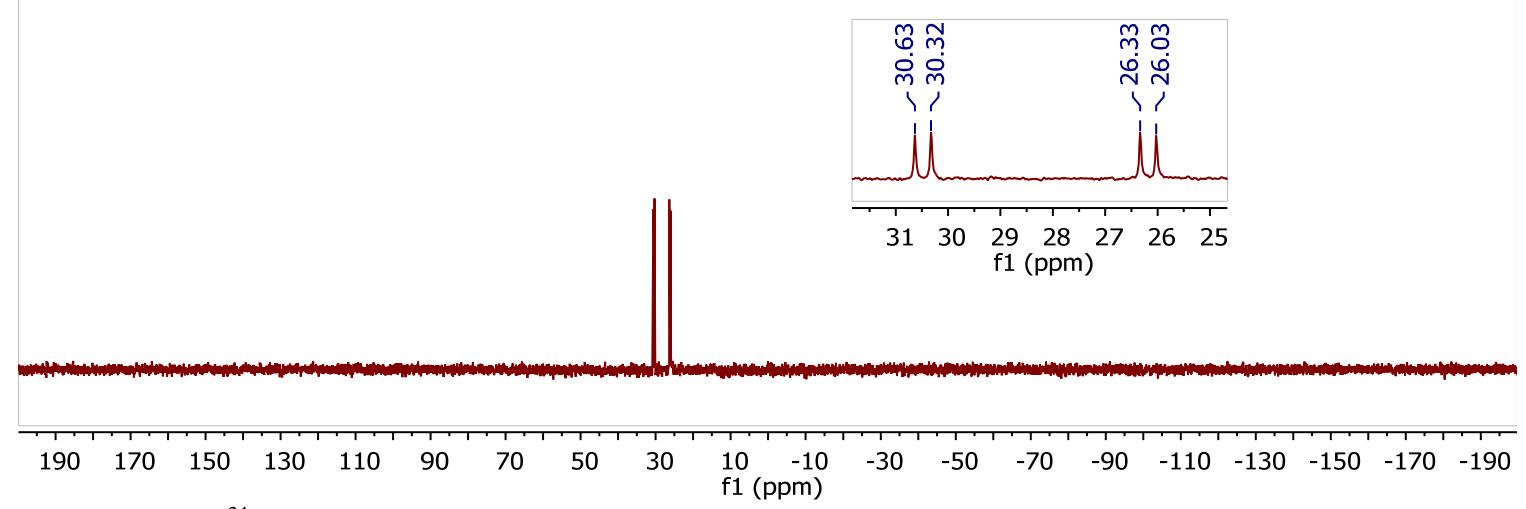

Figure S25. ${ }^{31} \mathrm{P}-\mathrm{NMR}\left(121 \mathrm{MHz}, \mathrm{CD}_{3} \mathrm{CN}\right)$ spectrum of $\mathbf{7 - H}$. 


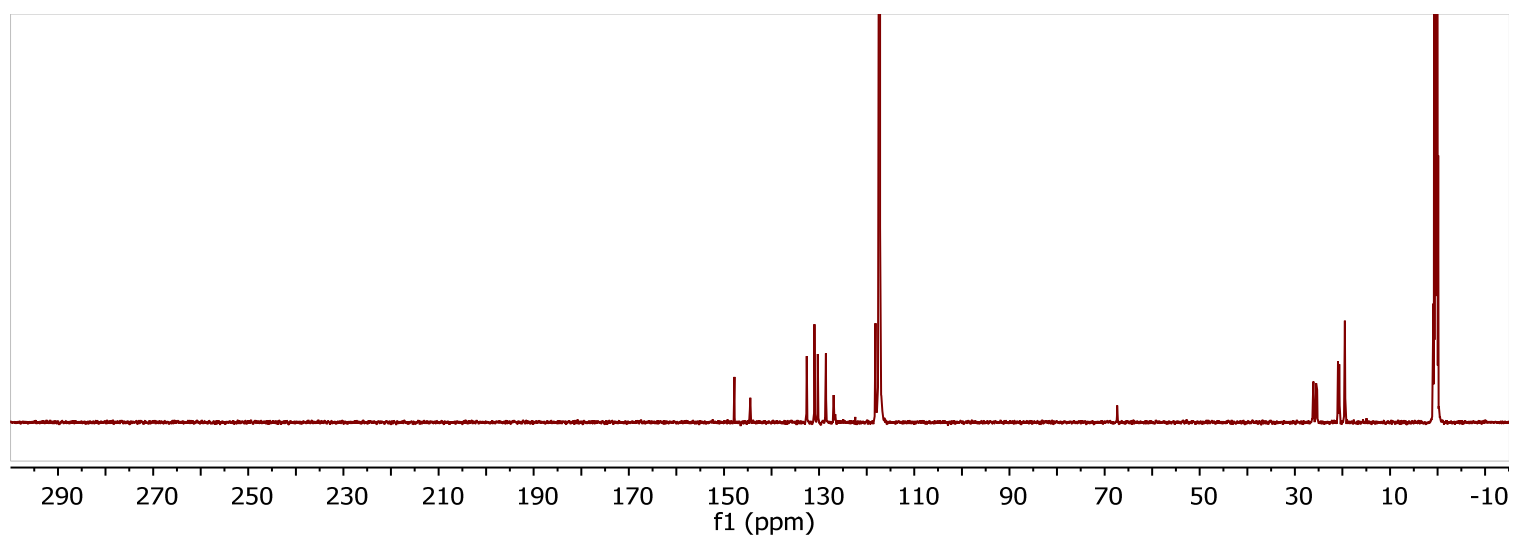

Figure S26. ${ }^{13} \mathrm{C}-\mathrm{NMR}\left(126 \mathrm{MHz}, \mathrm{CD}_{3} \mathrm{CN}\right)$ spectrum of $\mathbf{7 - H}$.

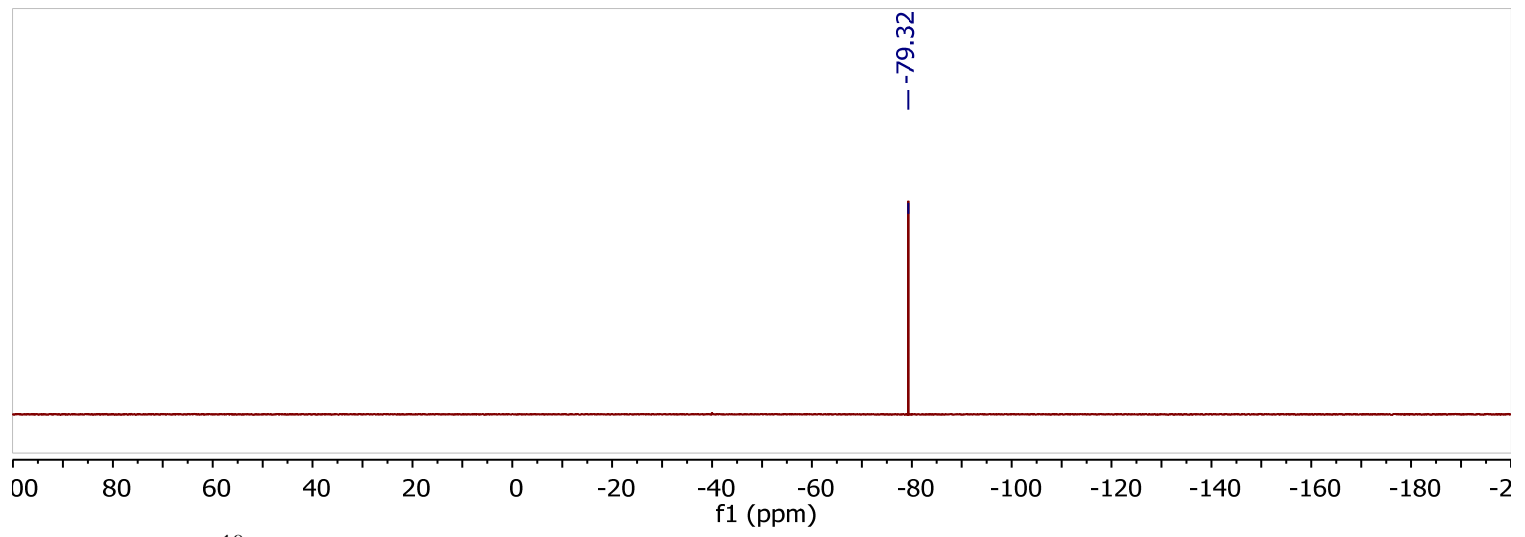

Figure S27. ${ }^{19} \mathrm{~F}-\mathrm{NMR}\left(282 \mathrm{MHz}, \mathrm{CD}_{3} \mathrm{CN}\right)$ spectrum of $\mathbf{7 - H}$.

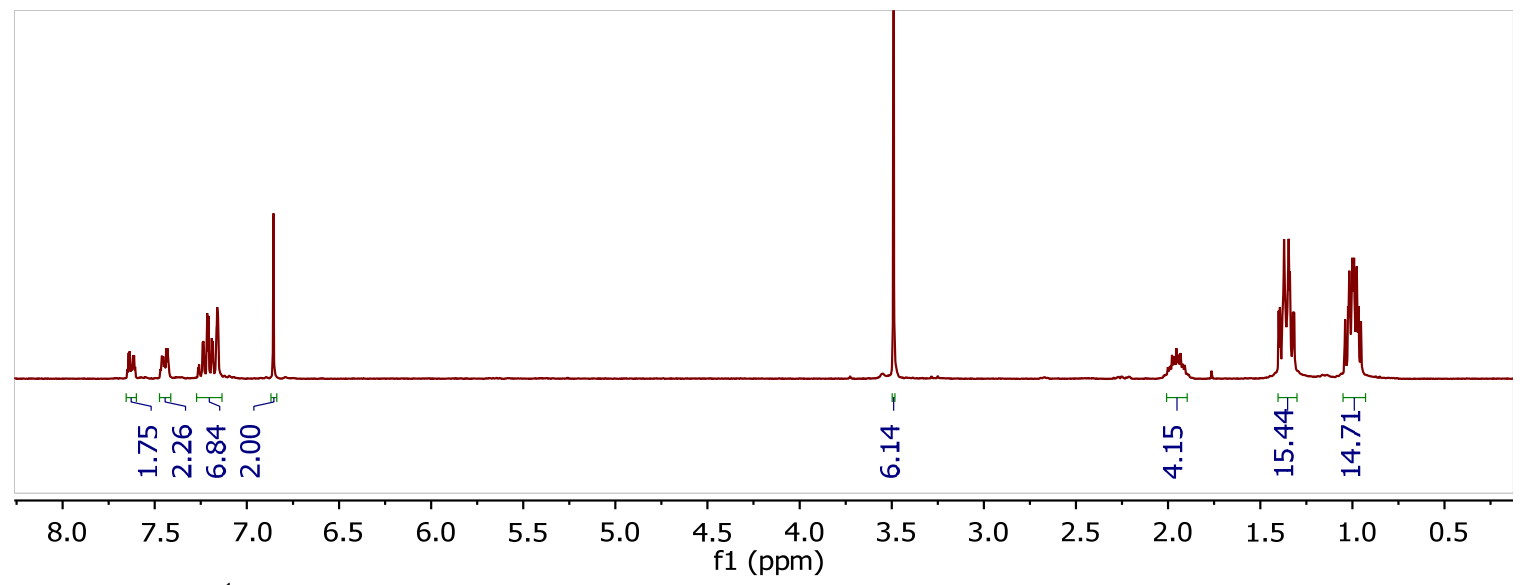

Figure S28. ${ }^{1} \mathrm{H}-\mathrm{NMR}\left(500 \mathrm{MHz}, \mathrm{C}_{6} \mathrm{D}_{6}\right)$ spectrum of 4-Me. 


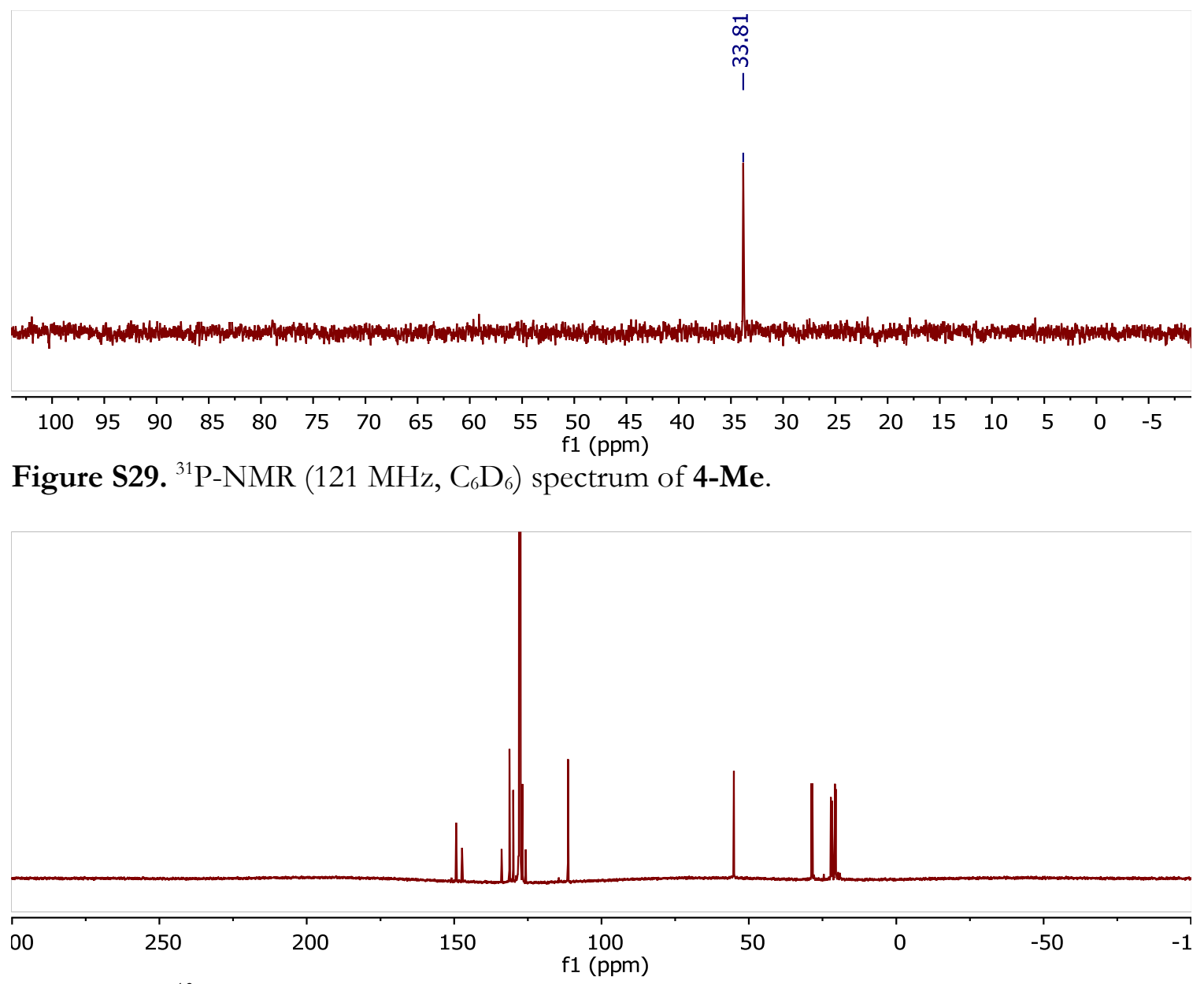

Figure S30. ${ }^{13} \mathrm{C}-\mathrm{NMR}\left(126 \mathrm{MHz}, \mathrm{C}_{6} \mathrm{D}_{6}\right)$ spectrum of 4-Me.

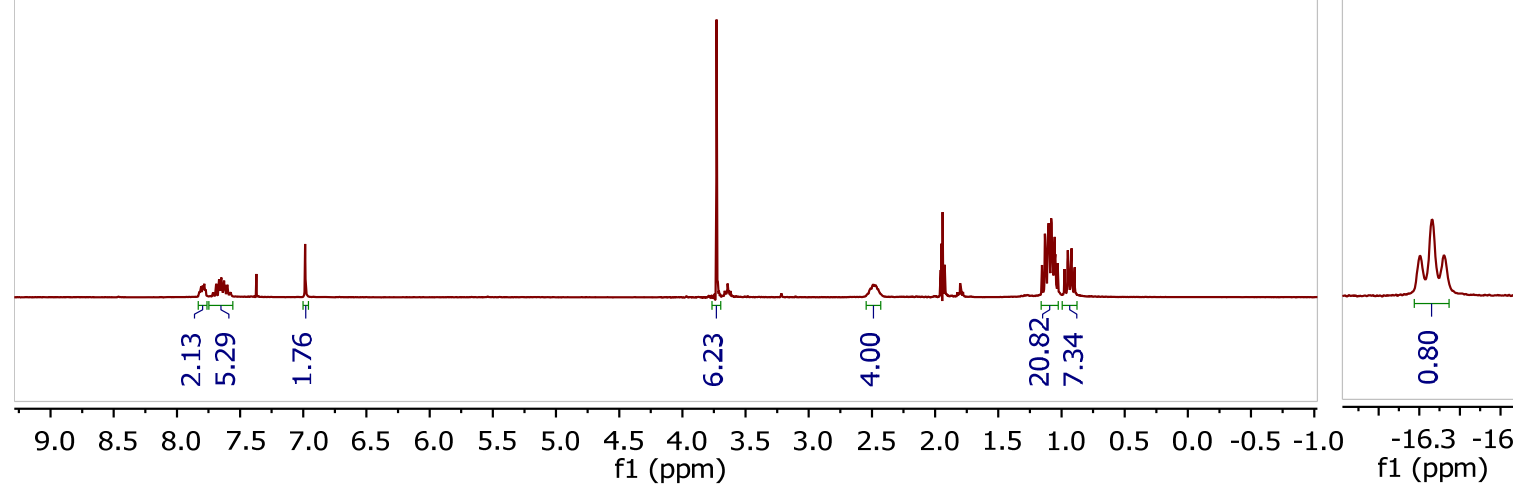

Figure S31. ${ }^{1} \mathrm{H}-\mathrm{NMR}\left(500 \mathrm{MHz}, \mathrm{CD}_{3} \mathrm{CN}\right)$ spectrum of 6-Me. 


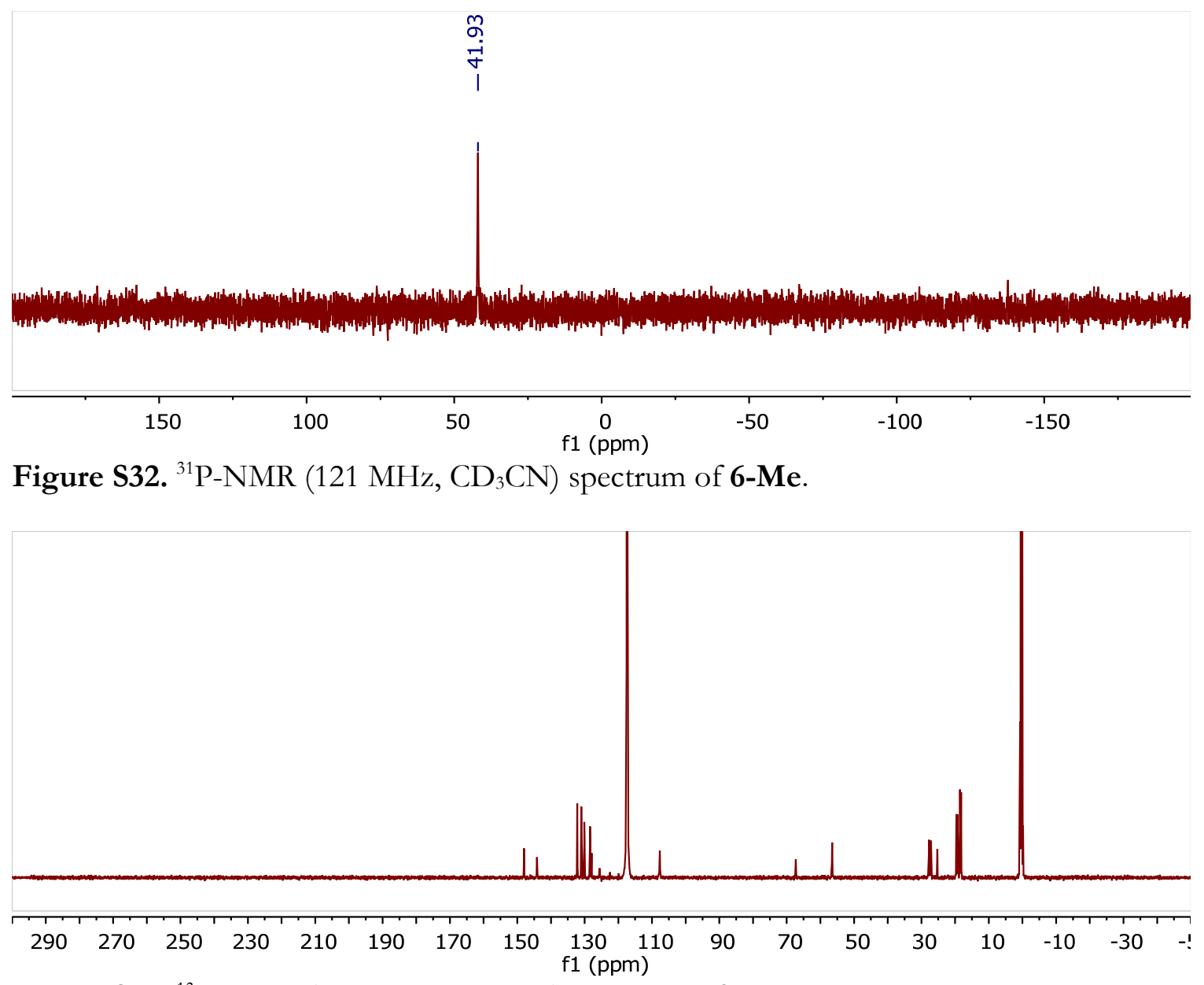

Figure S33. ${ }^{13} \mathrm{C}-\mathrm{NMR}\left(126 \mathrm{MHz}, \mathrm{CD}_{3} \mathrm{CN}\right)$ spectrum of 6-Me.

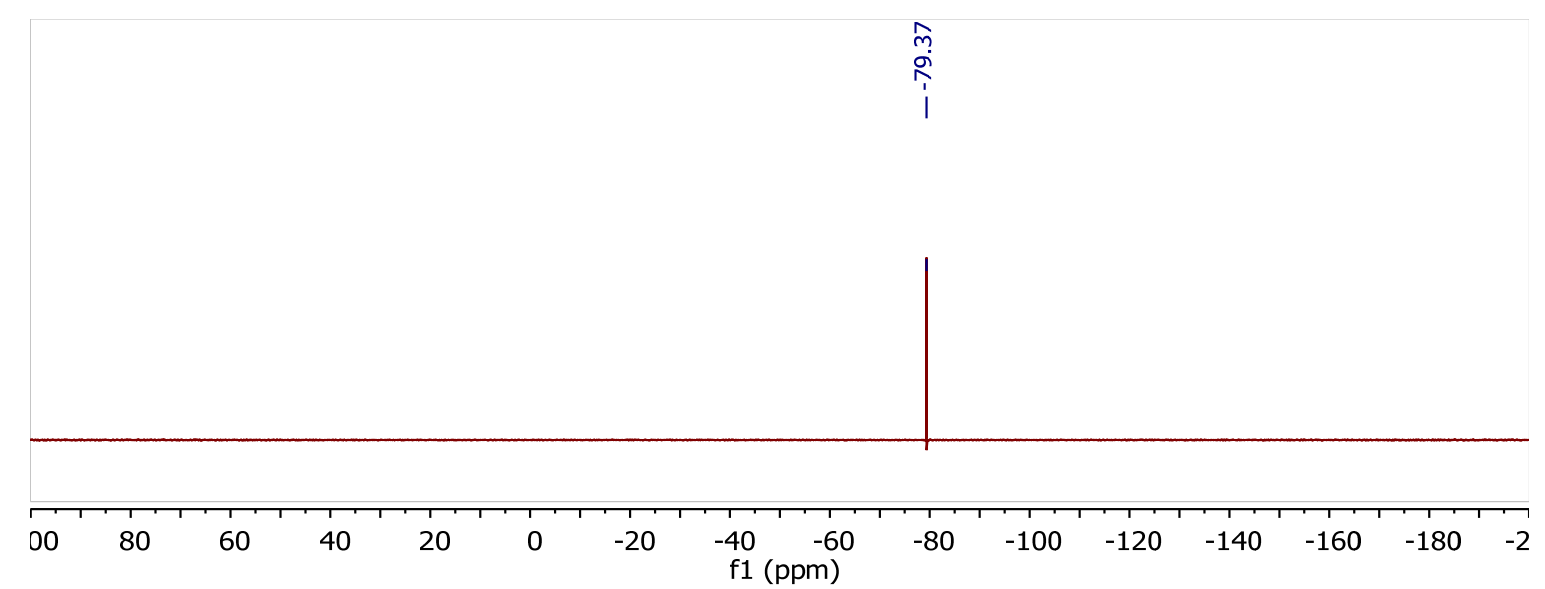

Figure S34. ${ }^{19} \mathrm{~F}-\mathrm{NMR}\left(282 \mathrm{MHz}, \mathrm{CD}_{3} \mathrm{CN}\right)$ spectrum of 6-Me. 


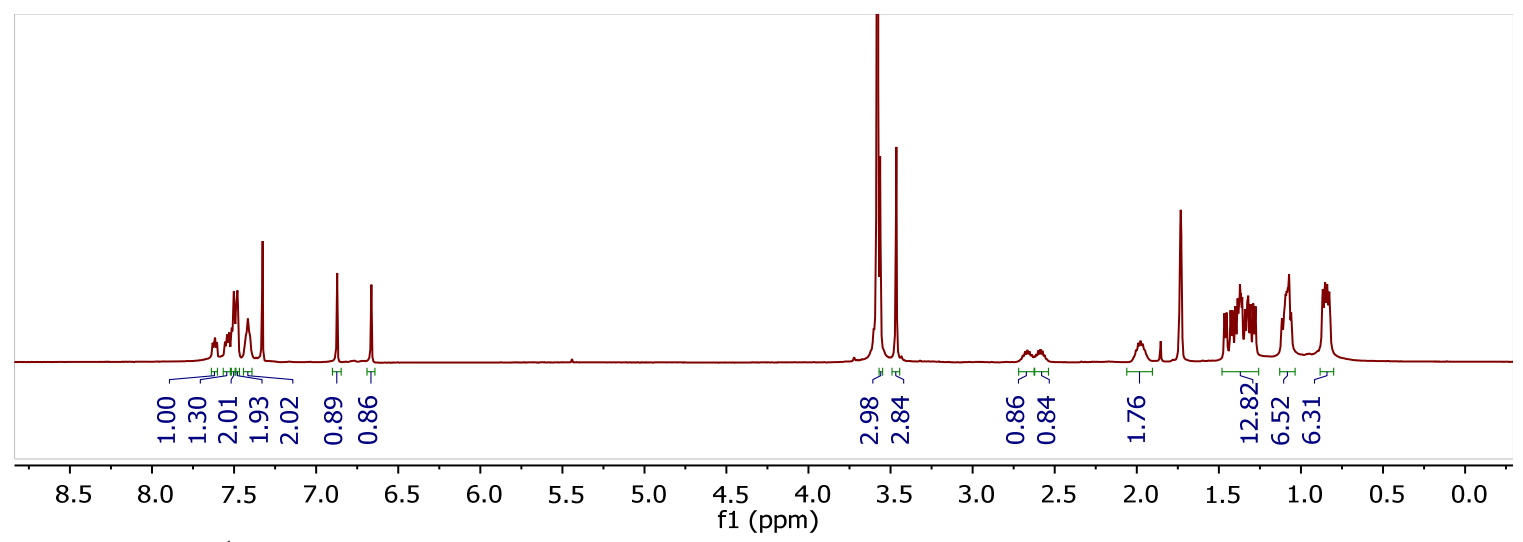

Figure S35. ${ }^{1} \mathrm{H}-\mathrm{NMR}\left(500 \mathrm{MHz}, \mathrm{d}_{8}\right.$-THF) spectrum of 8-Me.

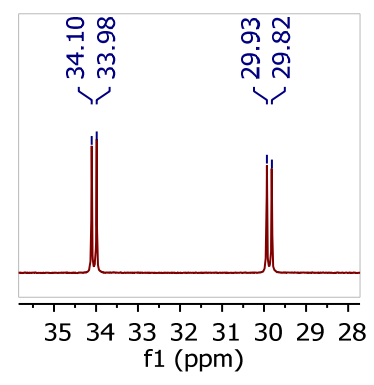

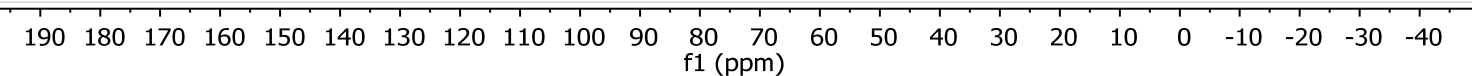

Figure S36. ${ }^{31}$ P-NMR $\left(202 \mathrm{MHz}, \mathrm{d}_{8}\right.$-THF) spectrum of 8-Me.

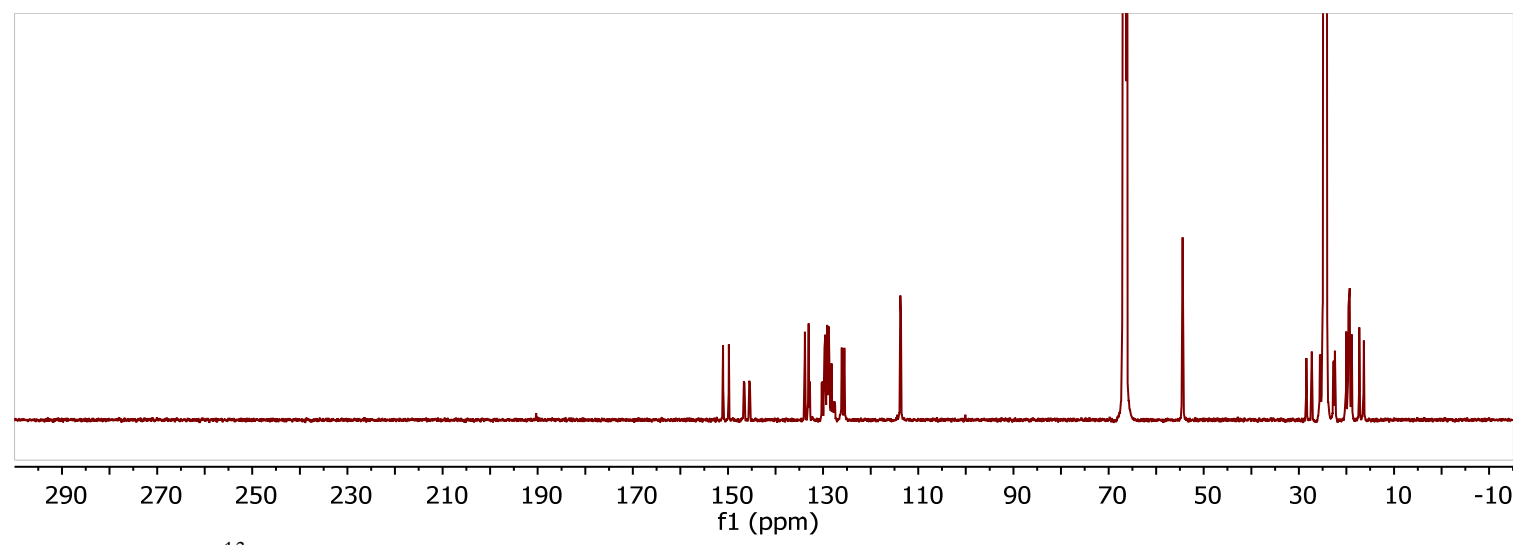

Figure S37. ${ }^{13} \mathrm{C}-\mathrm{NMR}\left(126 \mathrm{MHz}, \mathrm{d}_{8}\right.$-THF) spectrum of 8-Me. 


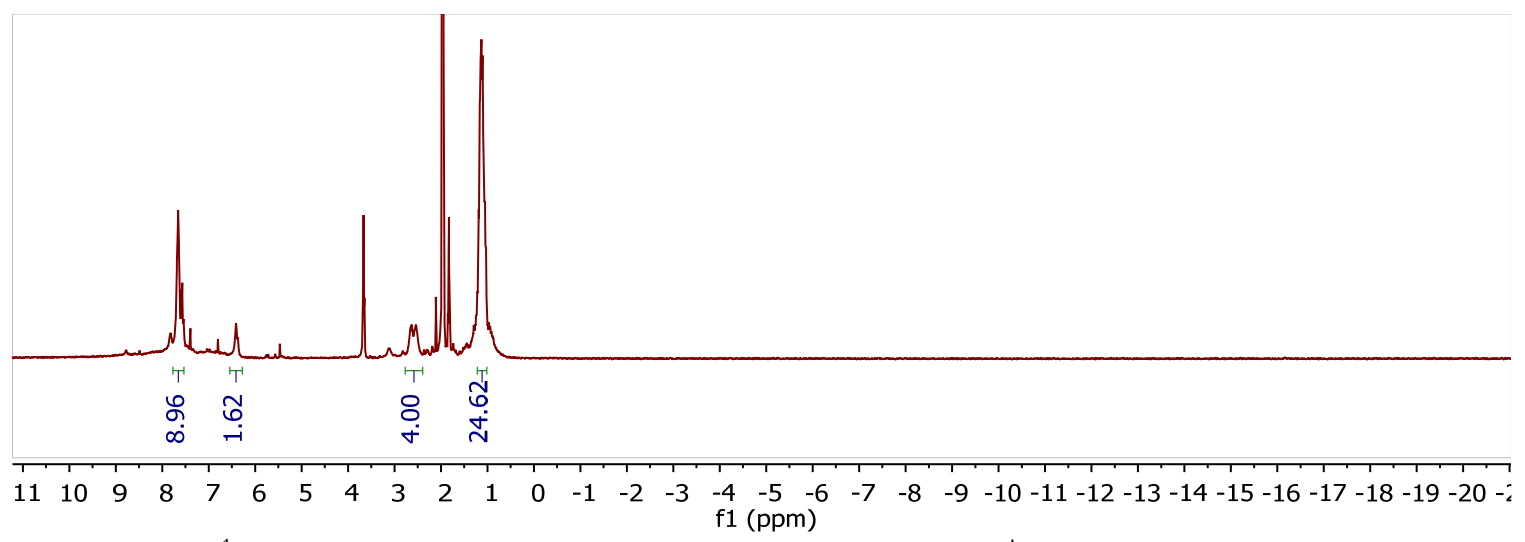

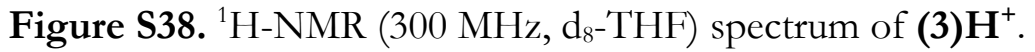

$$
\begin{aligned}
& \text { 우 } \\
& \text { Ð } \\
& 1
\end{aligned}
$$

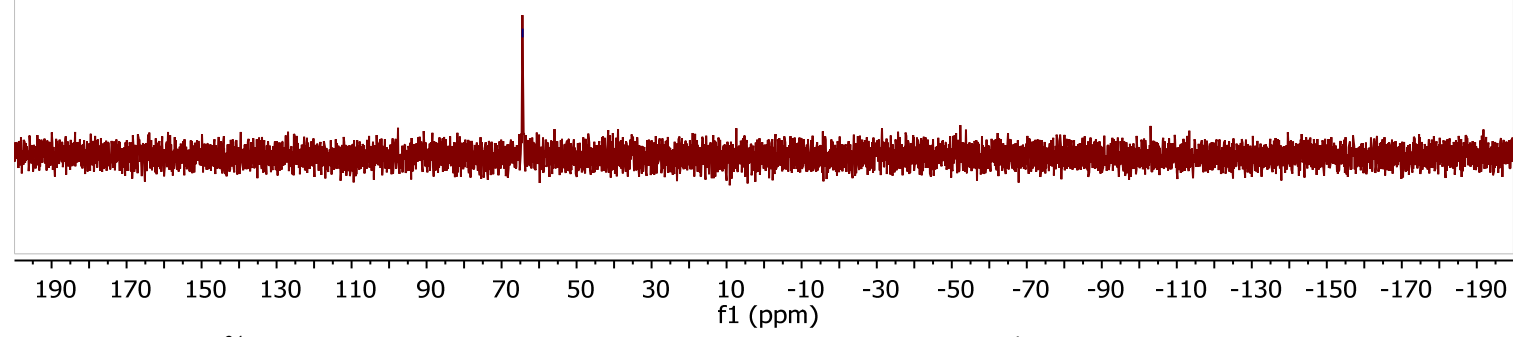

Figure S39. ${ }^{31}$ P-NMR (202 MHz, $\mathrm{d}_{8}$-THF) spectrum of $\mathbf{( 3 )} \mathbf{H}^{+}$.

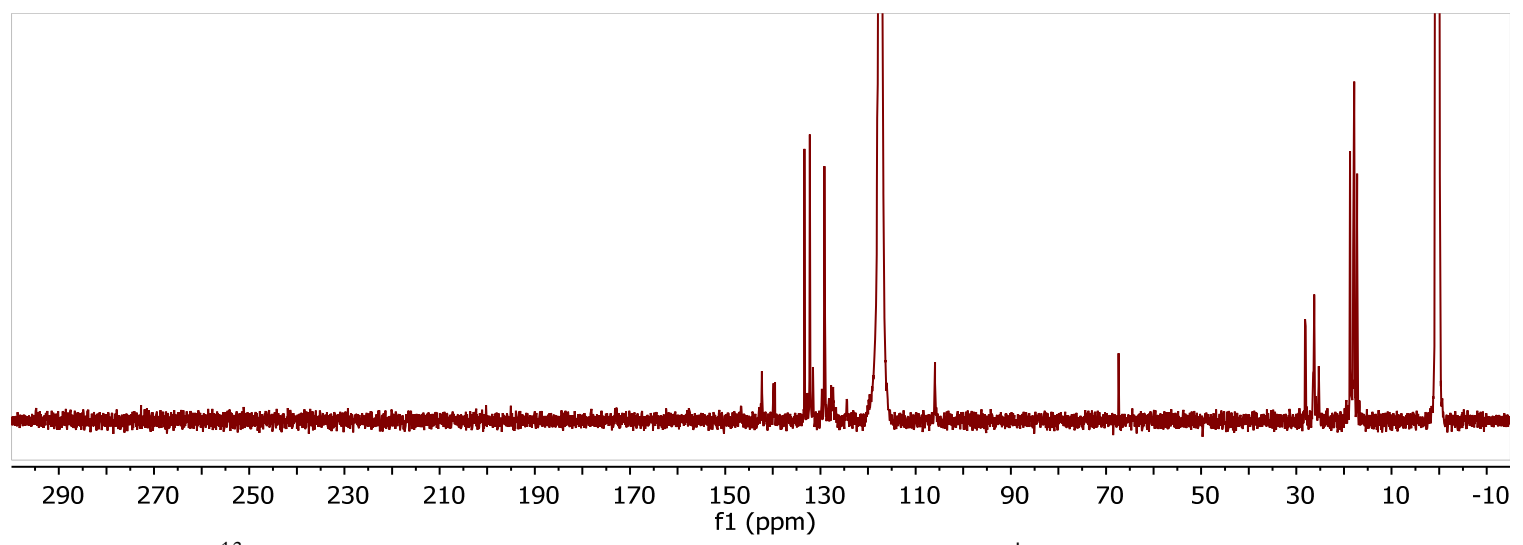

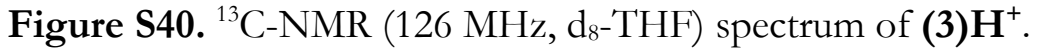




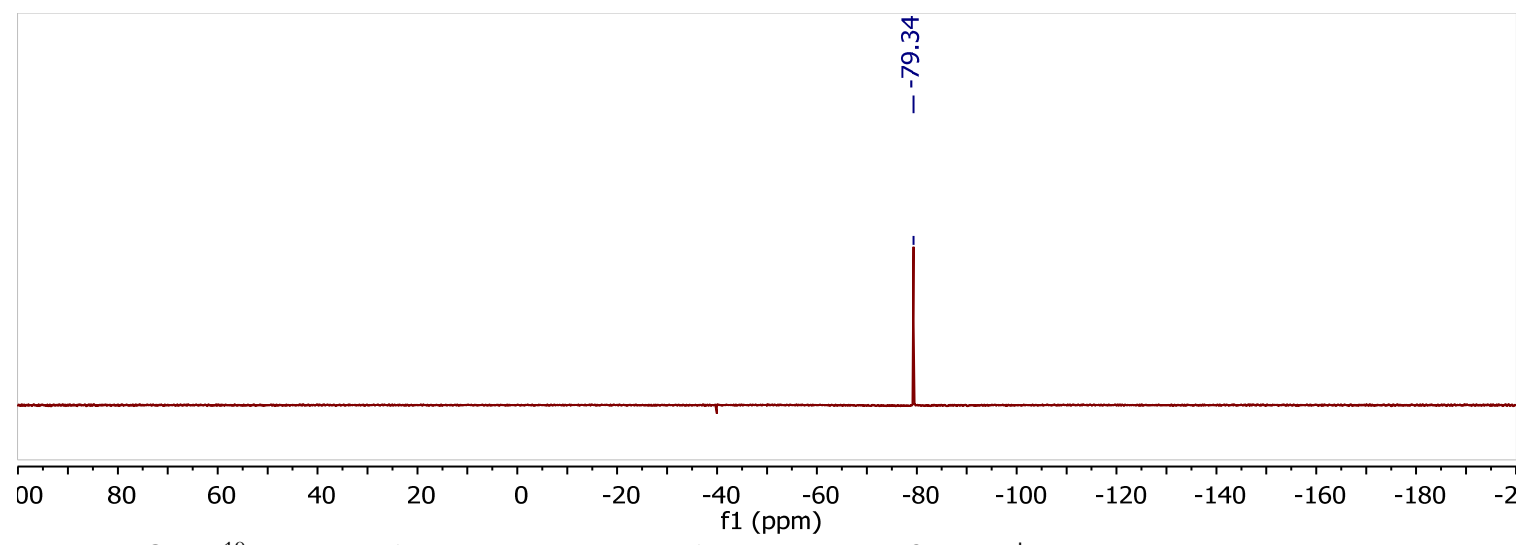

Figure S41. ${ }^{19} \mathrm{~F}-\mathrm{NMR}\left(282 \mathrm{MHz}, \mathrm{CD}_{3} \mathrm{CN}\right)$ spectrum of $(\mathbf{3}) \mathbf{H}^{+}$.

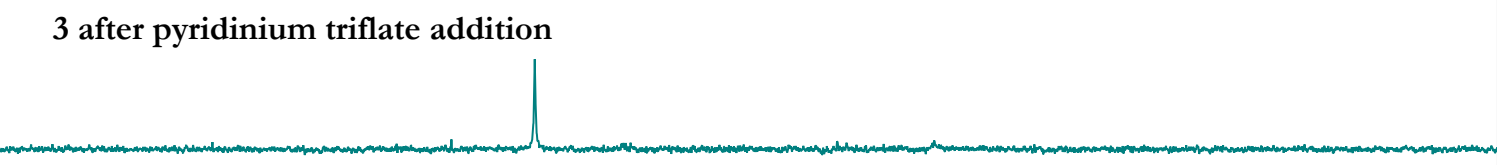

6-H after dioxygen addition

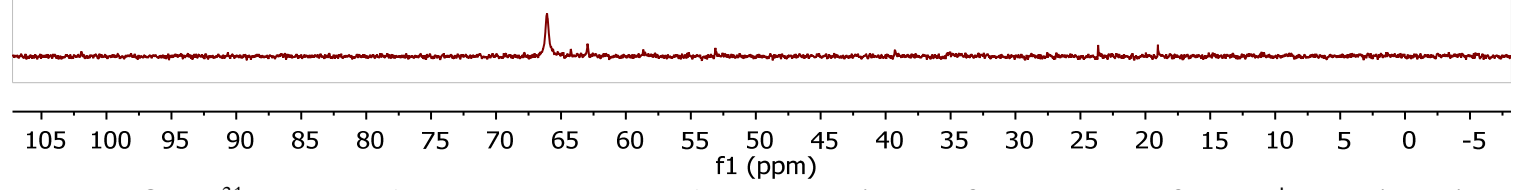

Figure S42. ${ }^{31} \mathrm{P}-\mathrm{NMR}\left(202 \mathrm{MHz}, \mathrm{CD}_{3} \mathrm{CN}\right)$. Comparison of spectrum of $(3) \mathbf{H}^{+}$. produced from 3 with pyridinium triflate (Top) and $\mathbf{6}-\mathbf{H}$ and dioxygen (Bottom). 


\section{Volumetric Measurement of $\mathbf{O}_{2}$ Consumption \\ Toepler Pump Experimental Details}

To determine the stoichiometry of reaction of $\mathrm{O}_{2}$ with compounds 4- $\mathrm{H}$, 4-Me, and 6-H, a solution of the compound to be tested was degassed via three freeze-pump-thaw cycles on a high vacuum line. While the solution (benzene for $\mathbf{4 - H}$, THF for 4-Me and $\mathbf{6 - H}$ ) was still frozen, a known amount of $\mathrm{O}_{2}(\sim 3-5$ equiv) was measured with a volumetric Schlenk bulb (33.28 $\mathrm{mL}$ or $43.48 \mathrm{~mL}$ ). The exact procedure then varied depending on the compound used. For $\mathbf{4}-\mathbf{H}$ and $\mathbf{6}-\mathbf{H}$, the solution of compound was then warmed to room temperature and while stirring vigorously the $\mathrm{O}_{2}$ was then added to the reaction mixture. For 4-Me, the solution was warmed to $-78{ }^{\circ} \mathrm{C}$ and while stirring vigorously the dioxygen was added to the reaction mixture while this temperature was maintained. All reactions were allowed to stir for $1 \mathrm{hr}$. During this time, the solution of $\mathbf{4 - H}$ and $\mathbf{6 - H}$ were observed to change color from brown/yellow and pale yellow to purple and pink respectively. No substantial color changed was observed following $\mathrm{O}_{2}$ addition to 4-Me at low temperature. The solution was again frozen, and the gas present in the headspace was measured by Toepler pump $(30.23 \mathrm{~mL}$ volume). Three freeze-pump-thaw cycles were carried out on the final reaction solution to recover all unreacted gases. Table S1 summarizes all the separate runs of volumetric measurements.

Table S1. Results of volumetric $\mathrm{O}_{2}$ measurements. Volumetric bulb volume $=33.28 \mathrm{~mL}^{\mathrm{a}}$ or $43.48 \mathrm{~mL}^{\mathrm{b}}$, Toepler pump volume $=30.25 \mathrm{~mL}$.

\begin{tabular}{cccccccc}
\hline Run & Cmpd & T (K) & $\begin{array}{c}\text { mmol } \\
\mathbf{C m p d}\end{array}$ & $\begin{array}{c}\mathbf{O}_{2} \text { added } \\
(\mathbf{m m H g})\end{array}$ & $\begin{array}{c}\text { Final } \mathbf{O}_{2} \\
(\mathbf{m m H g})\end{array}$ & $\begin{array}{c}\text { mmol } \mathbf{O}_{2} \\
\text { Consumed }\end{array}$ & $\begin{array}{c}\text { Equiv } \\
\text { Consumed }\end{array}$ \\
\hline $1^{\mathrm{a}}$ & $\mathbf{4 - H}$ & 295 & 0.0902 & 151 & 197 & 0.0507 & 0.56 \\
$2^{\mathrm{a}}$ & $\mathbf{4 - H}$ & 295 & 0.0928 & 152 & 197 & 0.0490 & 0.53 \\
$3^{\mathrm{b}}$ & $\mathbf{4 - H}$ & 295 & 0.0850 & 182 & 234 & 0.0454 & 0.53 \\
$4^{\mathrm{b}}$ & $\mathbf{6 - H}$ & 295 & 0.0808 & 186 & 224 & 0.0712 & 0.88 \\
$5^{\mathrm{b}}$ & $\mathbf{6 - H}$ & 295 & 0.0800 & 190 & 227 & 0.0758 & 0.95 \\
6 $^{\mathrm{b}}$ & $\mathbf{6 - H}$ & 295 & 0.0750 & 187 & 226 & 0.0703 & 0.94 \\
$7^{\mathrm{b}}$ & $\mathbf{4 - M e}$ & 295 & 0.0849 & 157 & 172 & 0.0882 & 1.04 \\
$8^{\mathrm{b}}$ & $\mathbf{4 - M e}$ & 295 & 0.0885 & 157 & 178 & 0.0783 & 0.89 \\
$9^{\mathrm{b}}$ & $\mathbf{4 - M e}$ & 295 & 0.0909 & 159 & 176 & 0.0863 & 0.95
\end{tabular}




\section{ReactIR Spectra}

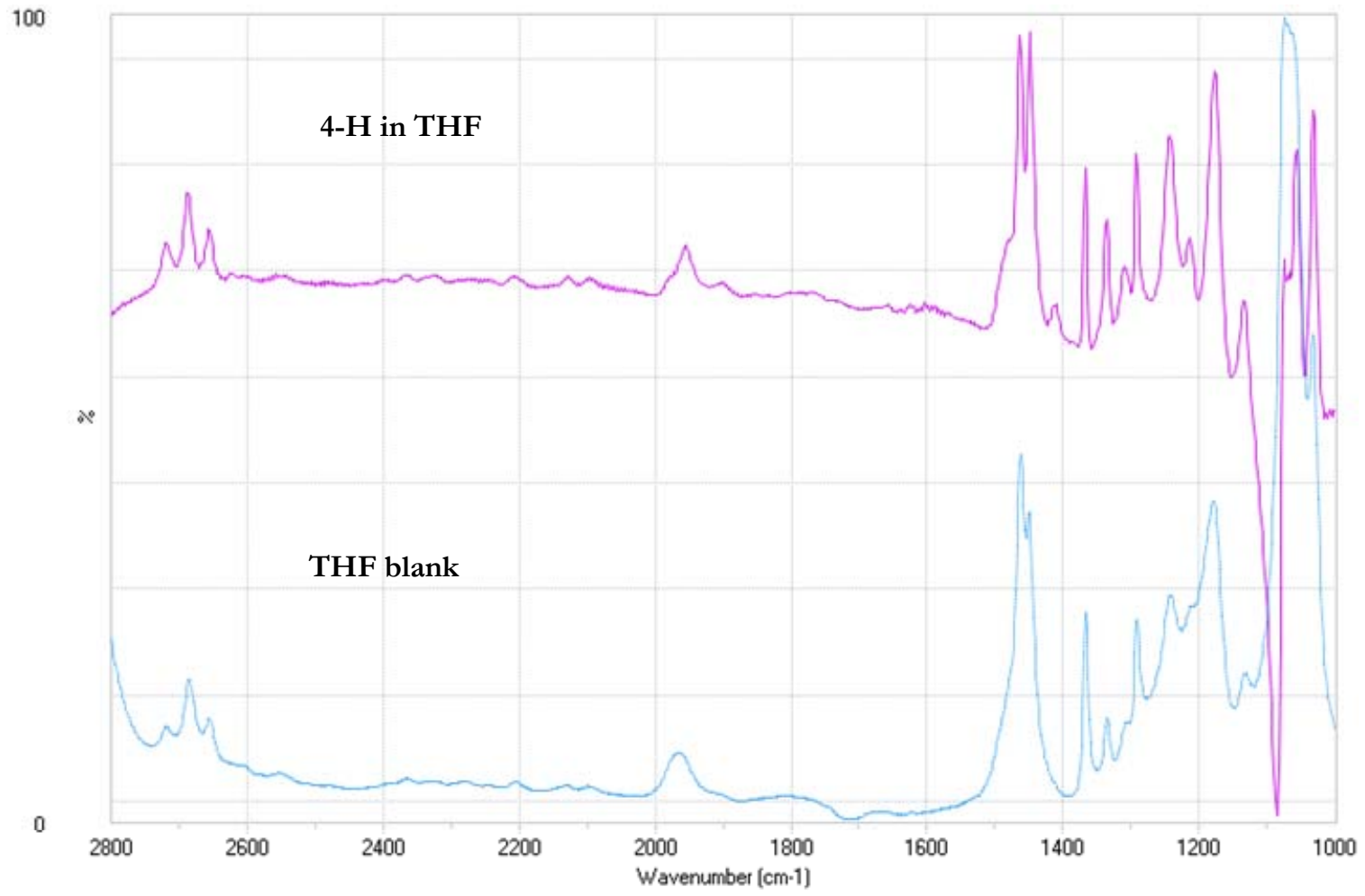

Figure S43. Solution ReactIR spectrum for $4-\mathbf{H}$ at $-78{ }^{\circ} \mathrm{C}$ before $\mathrm{O}_{2}$ addition.

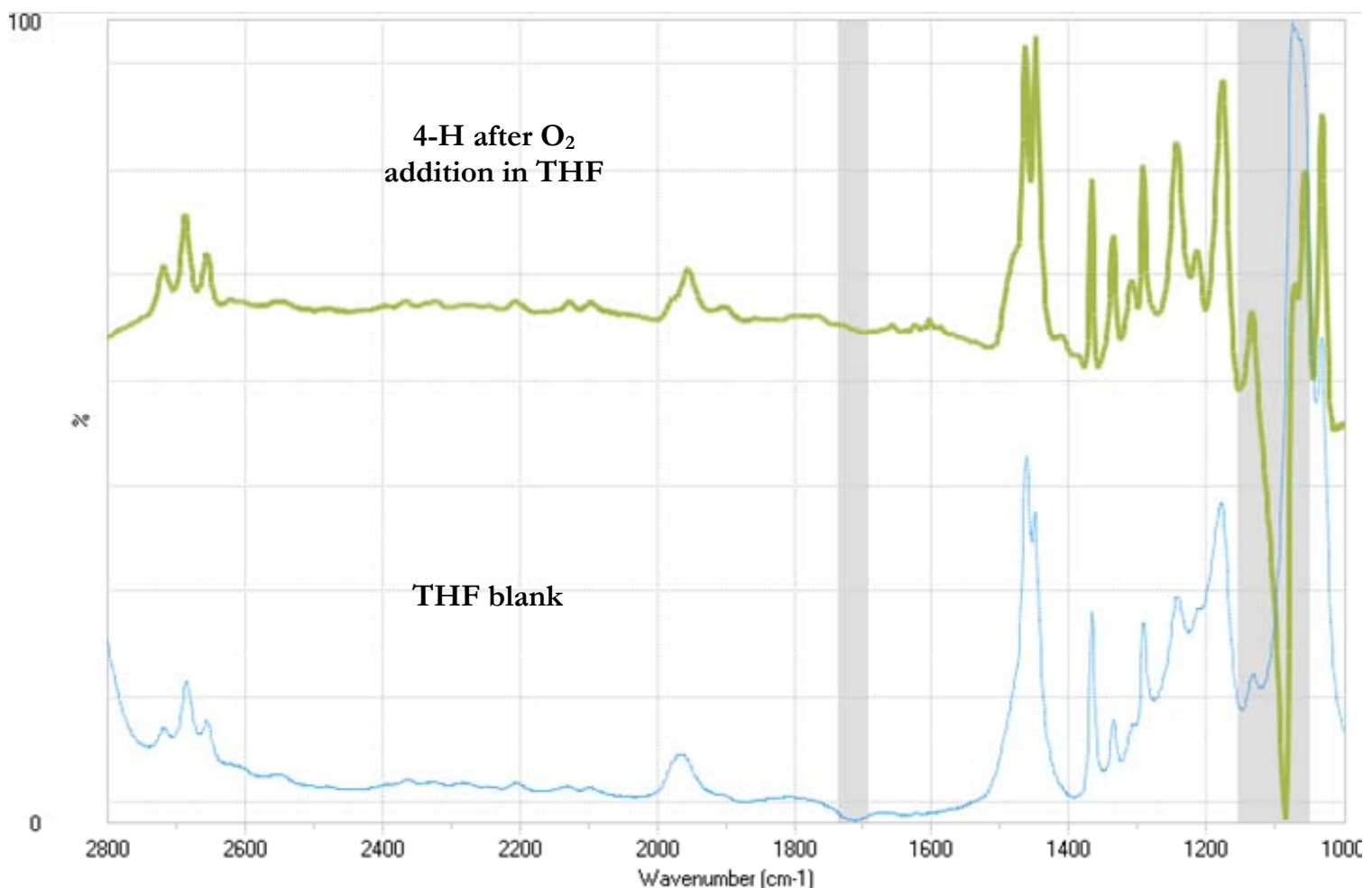

Figure S44. Solution ReactIR spectrum for $4-\mathrm{H}$ at $-78^{\circ} \mathrm{C}$ after $\mathrm{O}_{2}$ addition. 


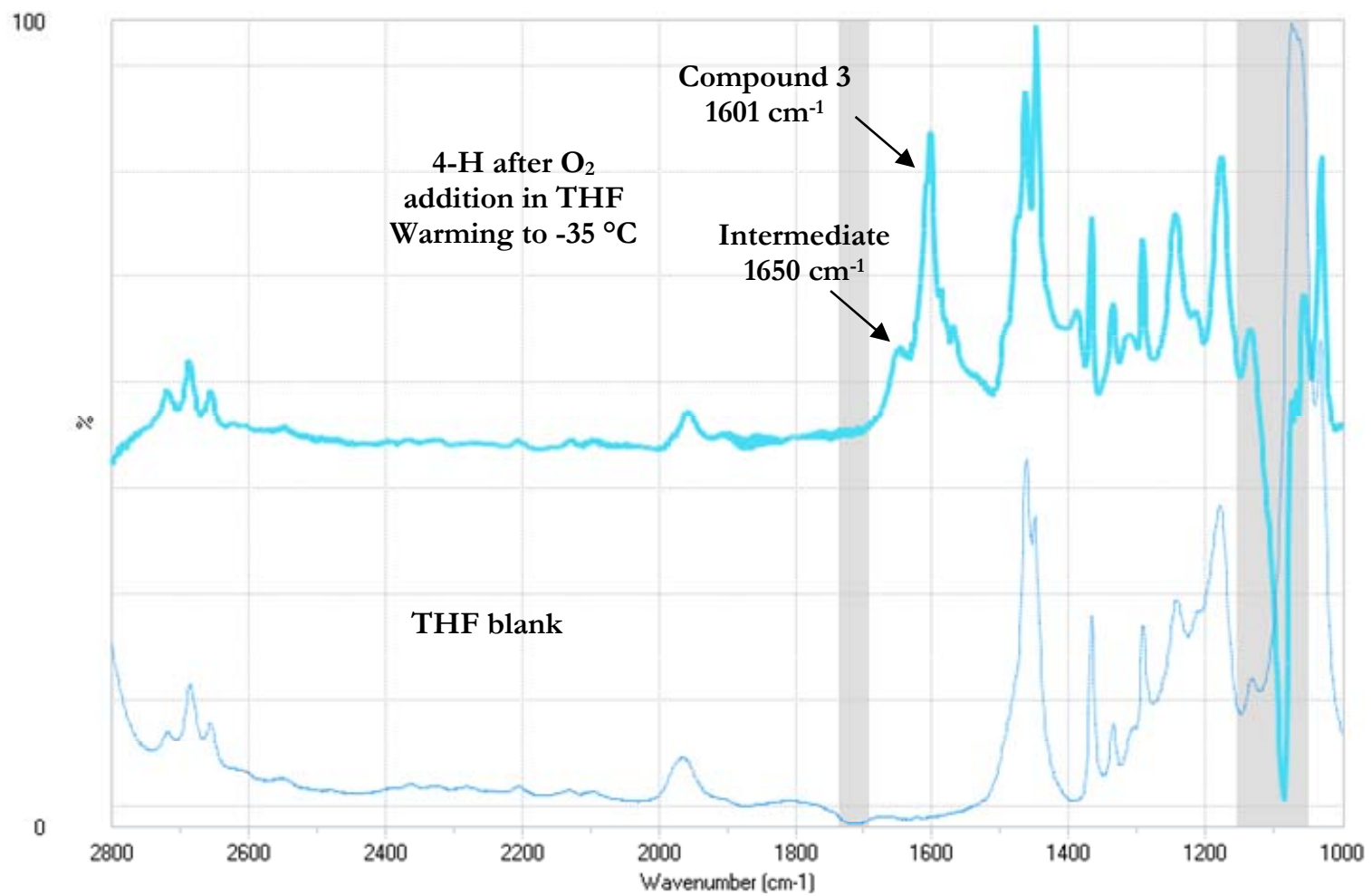

Figure S45. Solution ReactIR spectrum for $4-\mathrm{H}$ after warming to $-35{ }^{\circ} \mathrm{C}$ after $\mathrm{O}_{2}$ addition.

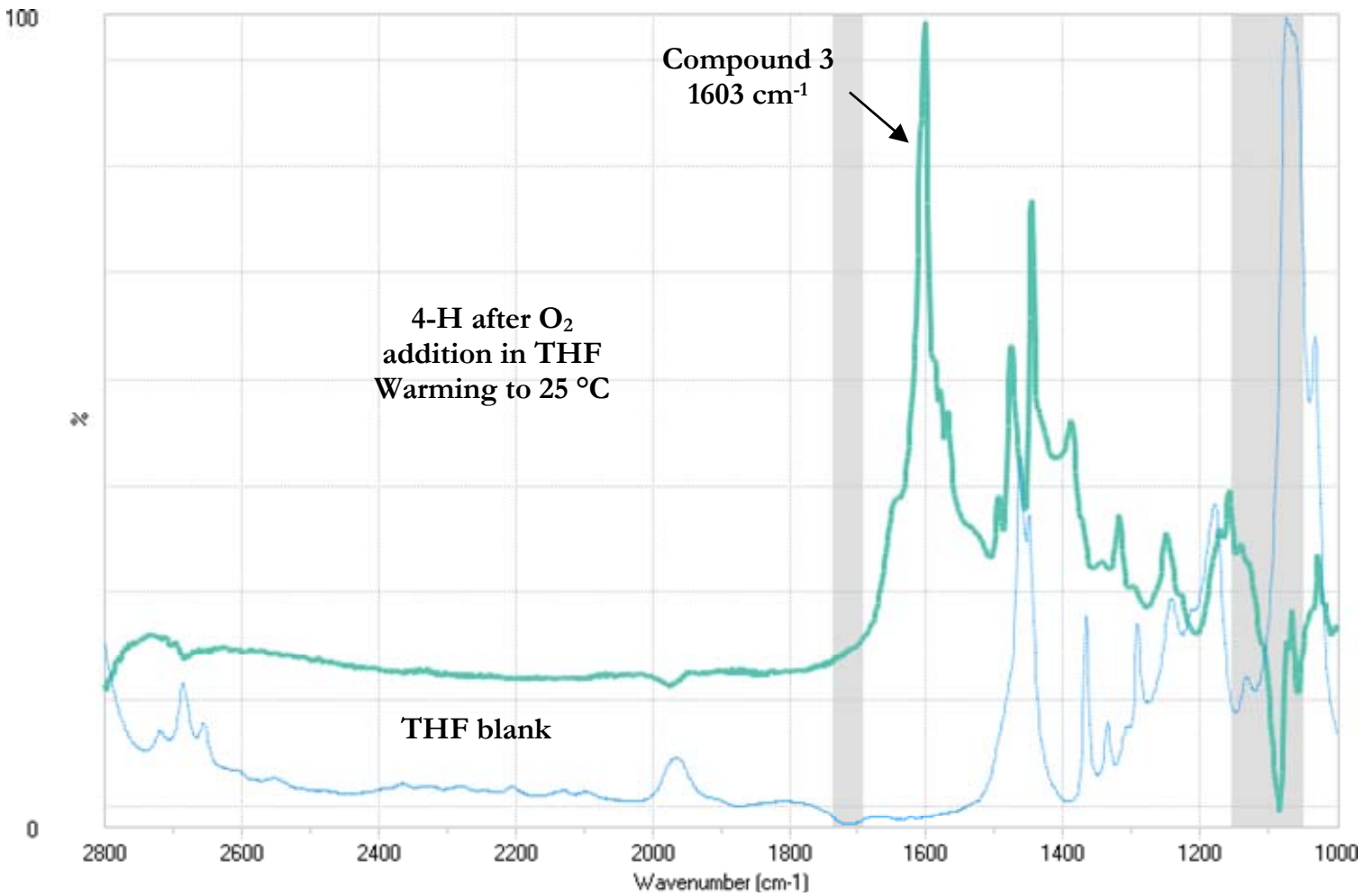

Figure S46. Solution ReactIR spectrum for $4-\mathrm{H}$ after warming to $25{ }^{\circ} \mathrm{C}$ after $\mathrm{O}_{2}$ addition. 
UV/Vis Spectra

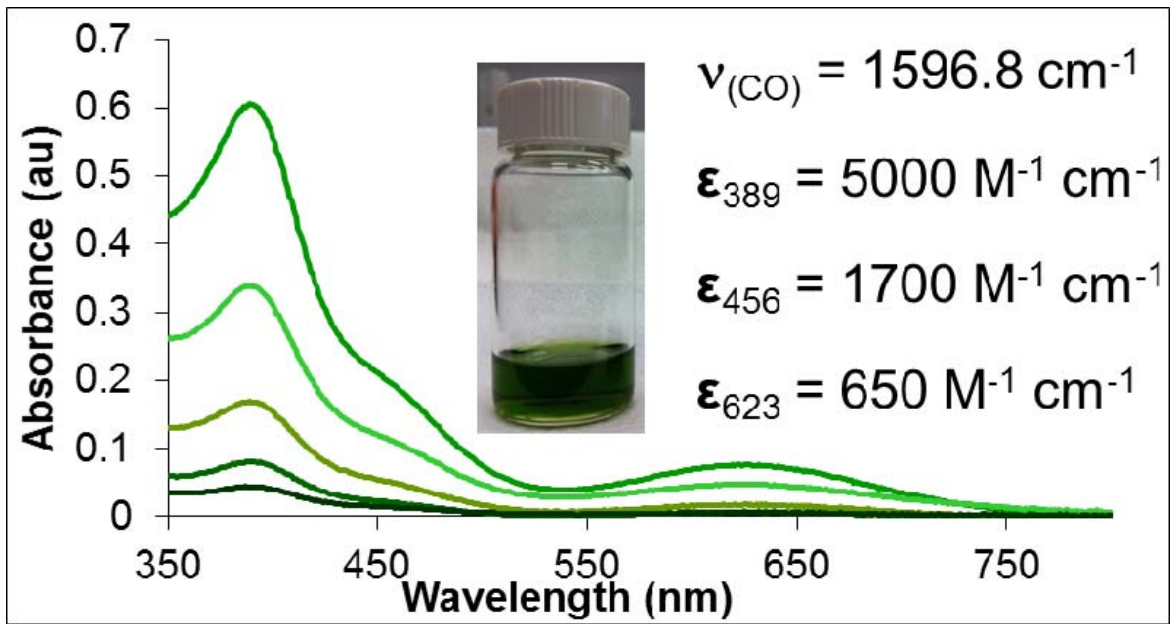

Figure S47. Solution UV/Vis spectrum for 2.

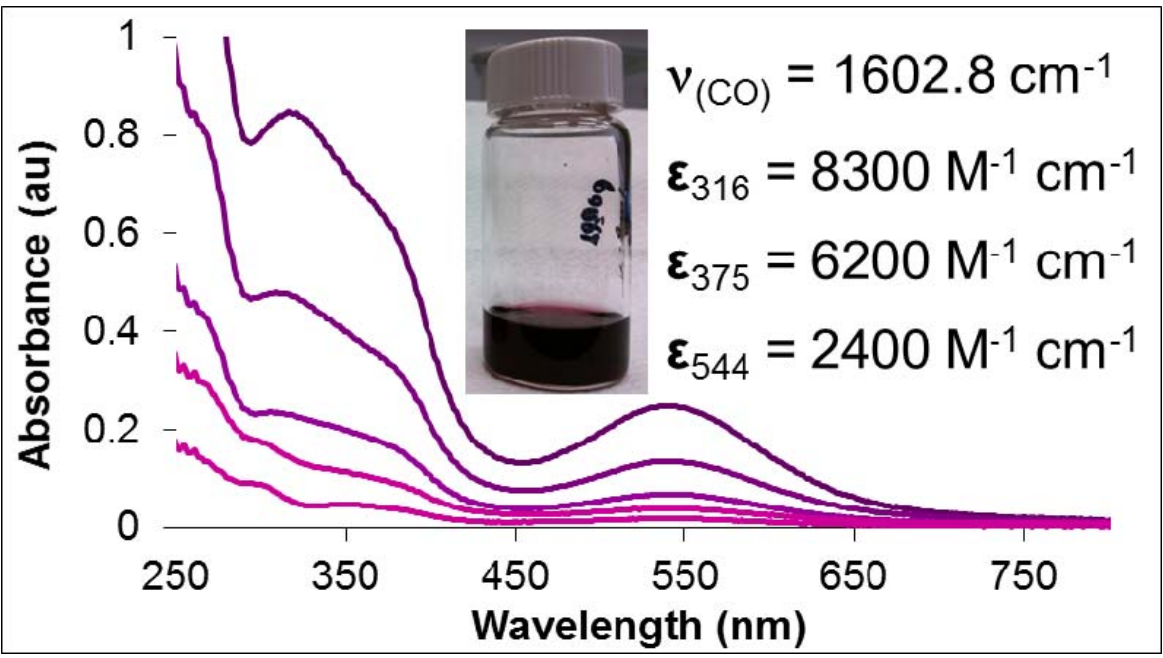

Figure S48. Solution UV/Vis spectrum for 3. 


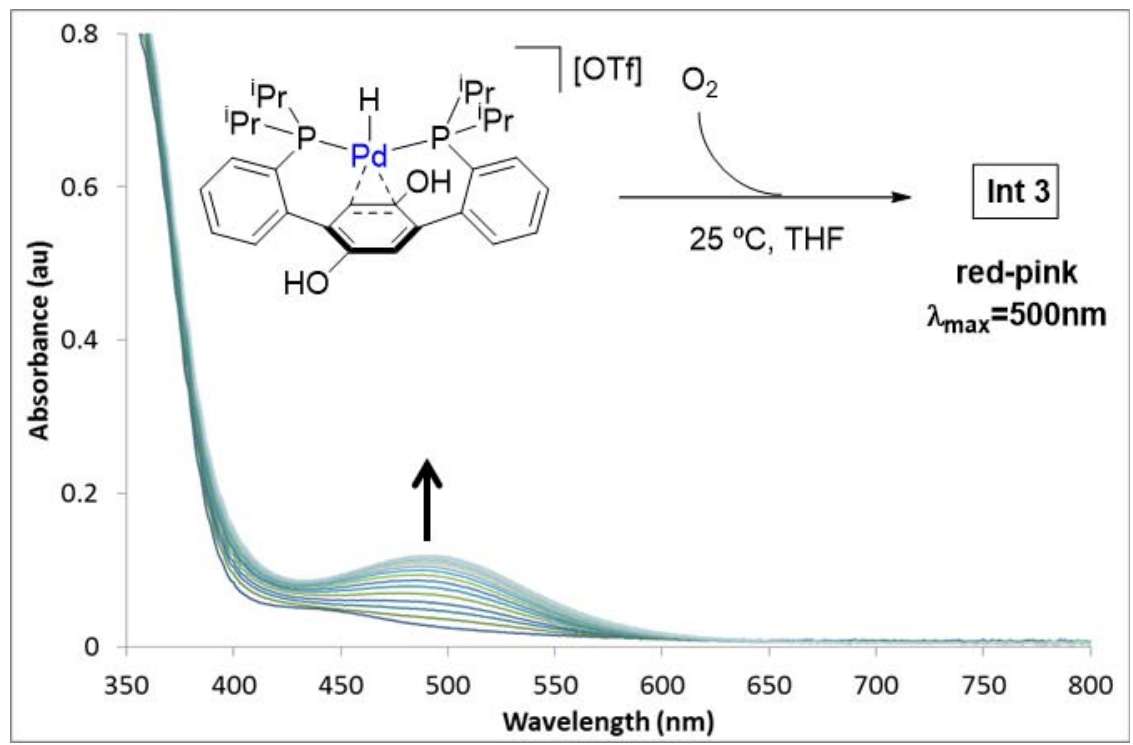

Figure S49. Solution UV/Vis spectrum for $\mathbf{6 - H}$ at $25{ }^{\circ} \mathrm{C}$ following $\mathrm{O}_{2}$ addition over multiple hours.

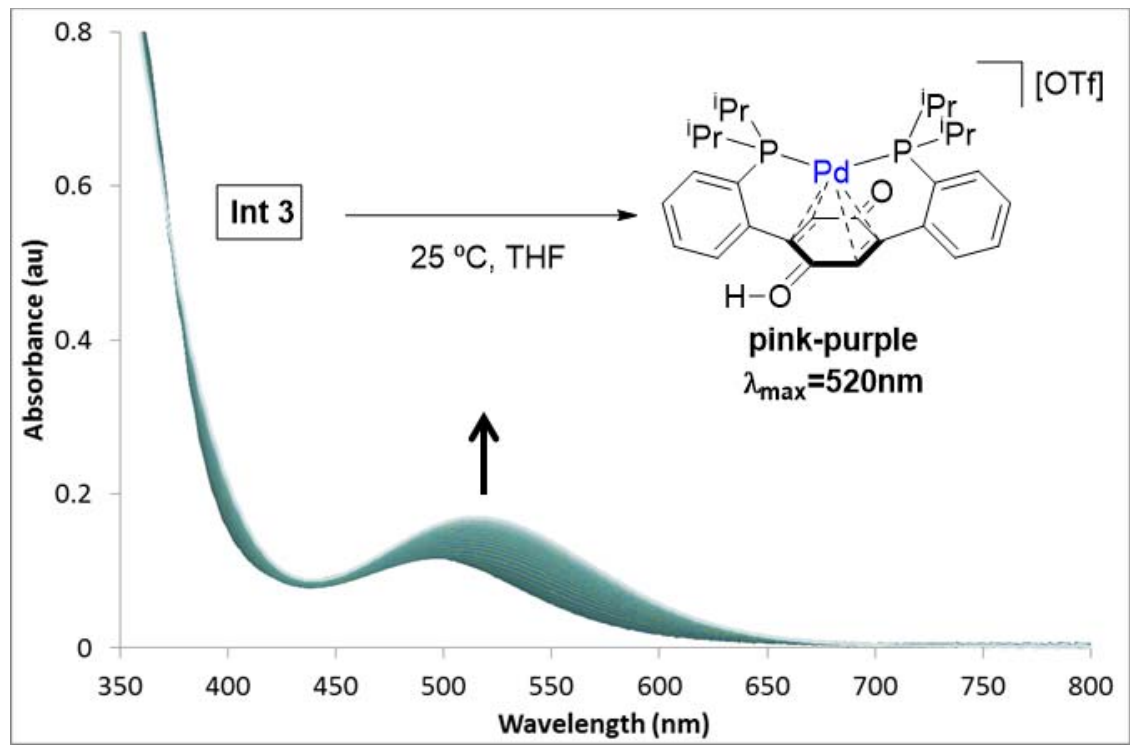

Figure S50. Solution UV/Vis spectrum for the conversion of Int3 to (3) $\mathbf{H}^{+}$after over multiple 12 hours.

\section{Oxidation of 4-H to 3 by Various Substrates \\ Dioxygen}

4-H (10.1 mg, $0.0170 \mathrm{mmol}, 1$ equiv) was transferred to a Schlenk tube as a solution in ca. 2 $\mathrm{mL}$ of THF. The solution was degassed by three freeze-pump-thaw cycles and then cooled to $-78{ }^{\circ} \mathrm{C}$. Excess $\mathrm{O}_{2}(1 \mathrm{~atm})$ was then added and allowed to stir vigorously for several minutes. The reaction mixture was then degassed under dynamic vacuum at $-78{ }^{\circ} \mathrm{C}$ while stirring vigorously for 8 minutes. The reaction mixture was then warmed to room temperature. During this time the solution immediately turned purple indicative of the formation of 3. Volatiles were then removed under reduced pressure and NMR of the residue taken in $\mathrm{C}_{6} \mathrm{D}_{6}$, which confirmed the quantitative formation of 3 . If the reaction is run 
in a J-Young tube in $\mathrm{C}_{6} \mathrm{D}_{6}$ the formation of water could be confirmed by vacuum transferring the volatiles of the reaction into another J-Young tube.

Nitric Oxide

4-H (10.0 mg, $0.0167 \mathrm{mmol}, 1$ equiv) was dissolved in $\mathrm{C}_{6} \mathrm{D}_{6}$ and transferred to a J-Young tube. The sample was frozen and the headspace removed. Nitric oxide (1 equiv) was measured in a calibrated gas bulb and then condensed into the J-Young tube. The sample was then warmed to room temperature. The solution turned purple as soon it thawed indicating the rapid formation of $\mathbf{3}$. This was confirmed by NMR which showed quantitative conversion to 3 . The formation of water could be confirmed by vacuum transferring the volatiles of the reaction into another J-Young tube.

Nitrous Oxide

4-H (9.9 mg, 0.0165 mmol, 1 equiv) was dissolved in $\mathrm{C}_{6} \mathrm{D}_{6}$ and transferred to a J-Young tube. The sample was frozen and the headspace removed. Excess nitrous oxide (1 atm) was then added to the J-Young tube. The sample was then warmed to room temperature. The reaction was then monitored by NMR. The slow formation of $\mathbf{3}$ was observed by NMR over the course of $12 \mathrm{hrs}$, however simultaneous decomposition of $\mathbf{4 - H}$ typical of solution samples allowed to stand has also occurred. A color change from brown-yellow to a purple was observed over the course of the reaction. The formation of water could be confirmed by vacuum transferring the volatiles of the reaction into another J-Young tube.

\section{2,4,6-Tritertbutyl Phenoxyl Radical}

4-H (10.0 mg, $0.0167 \mathrm{mmol}, 1$ equiv) was dissolved in ca. $1 \mathrm{~mL}$ of THF and transferred to a $20 \mathrm{~mL}$ scintillation vial equipped with a magnetic stirbar. 2,4,6-tritertbutyl phenoxyl radical ( $8.2 \mathrm{mg}, 0.033 \mathrm{mmol}, 2$ equiv) as then added as a solution in ca. $1 \mathrm{~mL}$ THF. This resulted in the immediate formation of a purple solution consistent with formation of $\mathbf{3}$. The volatiles were then removed under reduced pressure. Successful conversion to $\mathbf{3}$ was confirmed by ${ }^{31} \mathrm{P}$ NMR.

\section{1-Azido Adamantane}

4-H (10.6 mg, $0.0180 \mathrm{mmol}, 1$ equiv) was dissolved in ca. $1 \mathrm{~mL}$ of THF and transferred to a $20 \mathrm{~mL}$ scintillation vial equipped with a magnetic stirbar. 1-Azido adamantane $(3.2 \mathrm{mg}$, $0.0180 \mathrm{mmol}, 1$ equiv) was added as a solution in ca. $1 \mathrm{~mL}$ of THF and the reaction was allowed to stir for $2 \mathrm{hrs}$. During this time the solution turned from yellow-brown to purple, indicative of conversion to 3 . The volatiles were then removed under reduced pressure. Successful conversion to 3 was confirmed by ${ }^{31} \mathrm{P}$ NMR. The formation of 1-amino adamantine was confirmed by GC-MS comparisons to the retention time and mass data for an authentic sample.

\section{Trimethylamine n-oxide}

4-H (10.0 mg, $0.0166 \mathrm{mmol}, 1$ equiv) was dissolved in ca. $1 \mathrm{~mL}$ of $\mathrm{MeCN}$ and transferred to a $20 \mathrm{~mL}$ scintillation vial equipped with a magnetic stirbar. Trimethylamine $n$-oxide $(1.3 \mathrm{mg}$, $0.0173 \mathrm{mmol}, 1.04$ equiv) was added as a solution in ca. $1 \mathrm{~mL}$ of $\mathrm{MeCN}$ and the reaction was allowed to stir for $2 \mathrm{hrs}$. During this time the solution turned from yellow-brown to purple, indicative of conversion to 3 . The volatiles were then removed under reduced pressure. Successful conversion to $\mathbf{3}$ was confirmed by ${ }^{31} \mathrm{P}$ NMR. 
4-H (10.0 mg, $0.0166 \mathrm{mmol}, 1$ equiv) was dissolved in ca. $1 \mathrm{~mL}$ of THF and transferred to a $20 \mathrm{~mL}$ scintillation vial equipped with a magnetic stirbar. 1,4-Benzoquinone (1.8 mg, 0.167 mmol, 1 equiv) was then added as a solution in ca. $1 \mathrm{~mL}$ of THF and the reaction was allowed to stir for $2 \mathrm{hrs}$. During this time there was a rapid color change from brown-yellow to purple, indicative of conversion to 3 . The volatiles were then removed under reduced pressure. Successful conversion to 3 was confirmed by ${ }^{31} \mathrm{P}$ NMR.

\section{Representative Conditions for Testing Substrate Oxidation by 8-Me} Para-trifluorometbyl bensyl alcohol

In a Schlenk tube equipped with a magnetic stirbar, 4-Me $(11.3 \mathrm{mg}, 0.0180 \mathrm{mmol}, 1$ equiv) and 1,3,5-trimethoxybenzene $(3.7 \mathrm{mg}, 0.0180 \mathrm{mmol}, 1$ equiv), for an internal standard, was dissolved in ca. $5 \mathrm{~mL}$ of THF. The solution was degassed by a series of three freeze-pumpthaw cycles, then cooled to $-78{ }^{\circ} \mathrm{C}$. Excess $\mathrm{O}_{2}(1 \mathrm{~atm})$ was then added the solution was stirred vigorously to ensure complete conversion to 8-Me The solution was then degassed under dynamic vacuum at $-78{ }^{\circ} \mathrm{C}$ while stirring vigorously for several minutes. The reaction was then put under a $\mathrm{N}_{2}$ atmosphere and para-trifluoromethyl benzyl alcohol (24.6 $\mu \mathrm{L}, 0.180$ mmol, 10 equiv) was added via syringe. The reaction mixture was allowed to stir for $4 \mathrm{hrs}$ before a GC-MS aliquot was taken under a strong counterflow of $\mathrm{N}_{2}$. GC-MS indicated partial conversion to the corresponding benzaldehyde and unreacted benzyl alcohol by comparison to retention time and mass data for authentic samples. Addition of more equivalents of $\mathrm{O}_{2}$ did not lead to further conversion to product.

Para-trifluoromethyl-benzaldebyde

No oxidation of the benzaldehyde was observed by GC-MS.

Cyclohexane Carboxaldebyde

Partial conversion to cyclohexene observed by GC by comparison to retention time for an authentic sample.

Cyclohexene

No oxidation of cyclohexene was observed by GC. In particular, no cyclohexanone was observed by comparison to retention time for an authentic sample.

\section{Triphenylphosphine}

Partial conversion to triphenylphosphine oxide observed by GC-MS by comparison to retention time and MS data for an authentic sample. However, low temperature ${ }^{31} \mathrm{P}$ NMR experiments indicate that substitution of 1-Me by 2 equivalents of $\mathrm{PPh}_{3}$ to yield $\left(\mathrm{PPh}_{3}\right)_{2} \mathrm{Pd}\left(\eta^{2}-\mathrm{O}_{2}\right)$ occurs $\left({ }^{31} \mathrm{P}\right.$ shift observed at $33.18 \mathrm{ppm}$ consistent with literature data obtained in DMF (33.4 ppm) or $\mathrm{CDCl}_{3}(33.2 \mathrm{ppm})$ ). By comparison to the ${ }^{31} \mathrm{P}$ integration of against an internal standard of $\mathrm{OP}(\mathrm{OMe})_{3}$, the disappearance of 8-Me occurs at the same rate of 1-Me and $\left(\mathrm{PPh}_{3}\right)_{2} \mathrm{Pd}\left(\eta^{2}-\mathrm{O}_{2}\right)$ formation. Subsequent formation of phosphine oxide observed by GC-MS likely occurs from this complex rather than 8-Me. 


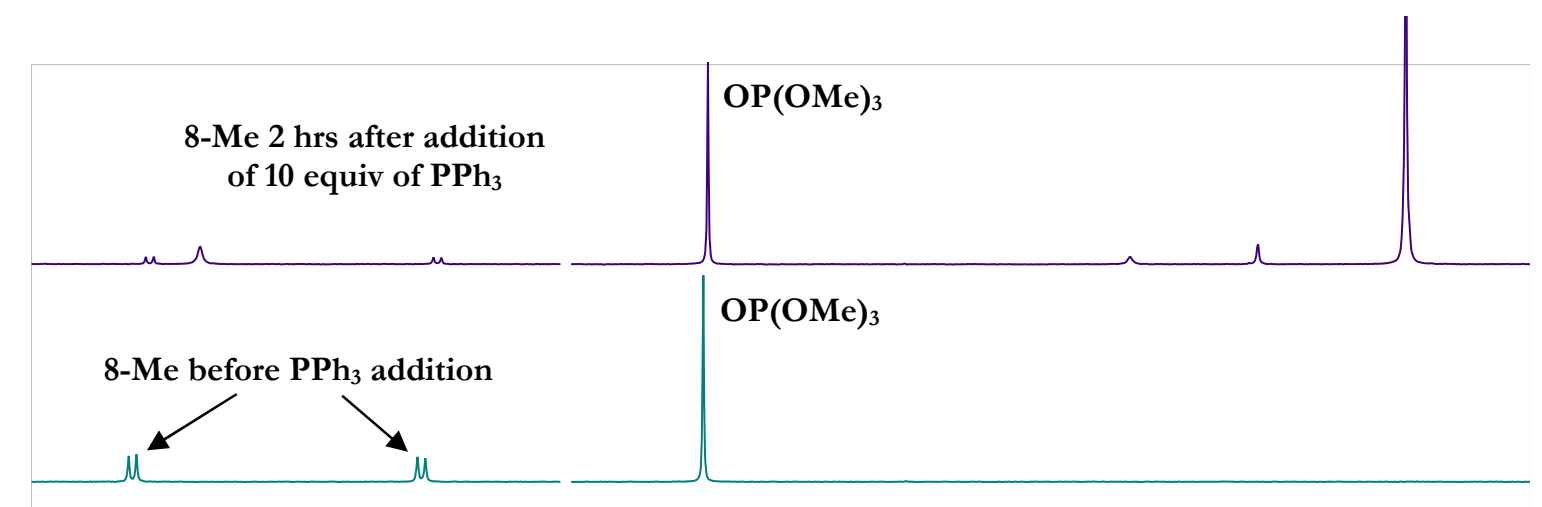

$\left(\mathrm{PPh}_{3}\right)_{2} \mathbf{P d}\left(\eta^{2}-\mathrm{O}_{2}\right)$ formed by $\mathrm{O}_{2}$

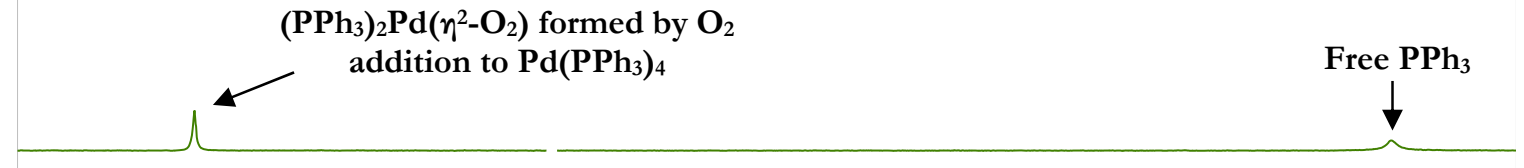

Atropisomers of 1-Me

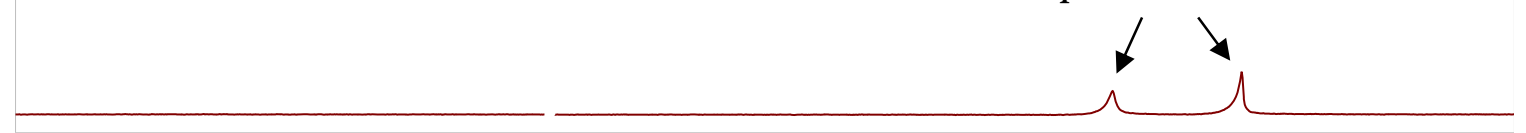

$\begin{array}{llllllllllllllllllllllllllll}35 & 34 & 33 & 32 & 31 & 30 & 29 & 28 & 5 & 4 & 3 & 2 & 1 & 0 & -1 & -2 & -3 & -4 & -5 & -6 & -7 & -8 & -9\end{array}$

Figure S51. NMR spectra taken at $-78{ }^{\circ} \mathrm{C}$ in THF confirming the substitution of $\mathrm{PPh}_{3}$ for 1Me in solution in compound 8-Me.

Metbyl-para-tolyl sulfide

No oxidation of the aryl-alkyl sulfide was observed. 


\section{Cyclic Voltammetry Data}

\section{Electrochemical Details}

Electrochemical measurements of complex 2 and $\mathbf{3}$ were taken on a Pine Instrument Company biopotentiostat model AFCBP1 as 3 millimolar solutions in tetrahydrofuran using 0.1 molar $\left[n \mathrm{Bu}_{4} \mathrm{~N}\right]\left[\mathrm{PF}_{6}\right]$ as the electrolyte with a platinum wire counter electrode, a glassy carbon working electrode, and a silver/silver nitrate reference electrode in 0.1 molar $\left[n \mathrm{Bu}_{4} \mathrm{~N}\right]\left[\mathrm{PF}_{6}\right]$ as an acetonitrile solution. Electrochemical measurements of compounds were internally referenced to ferrocene

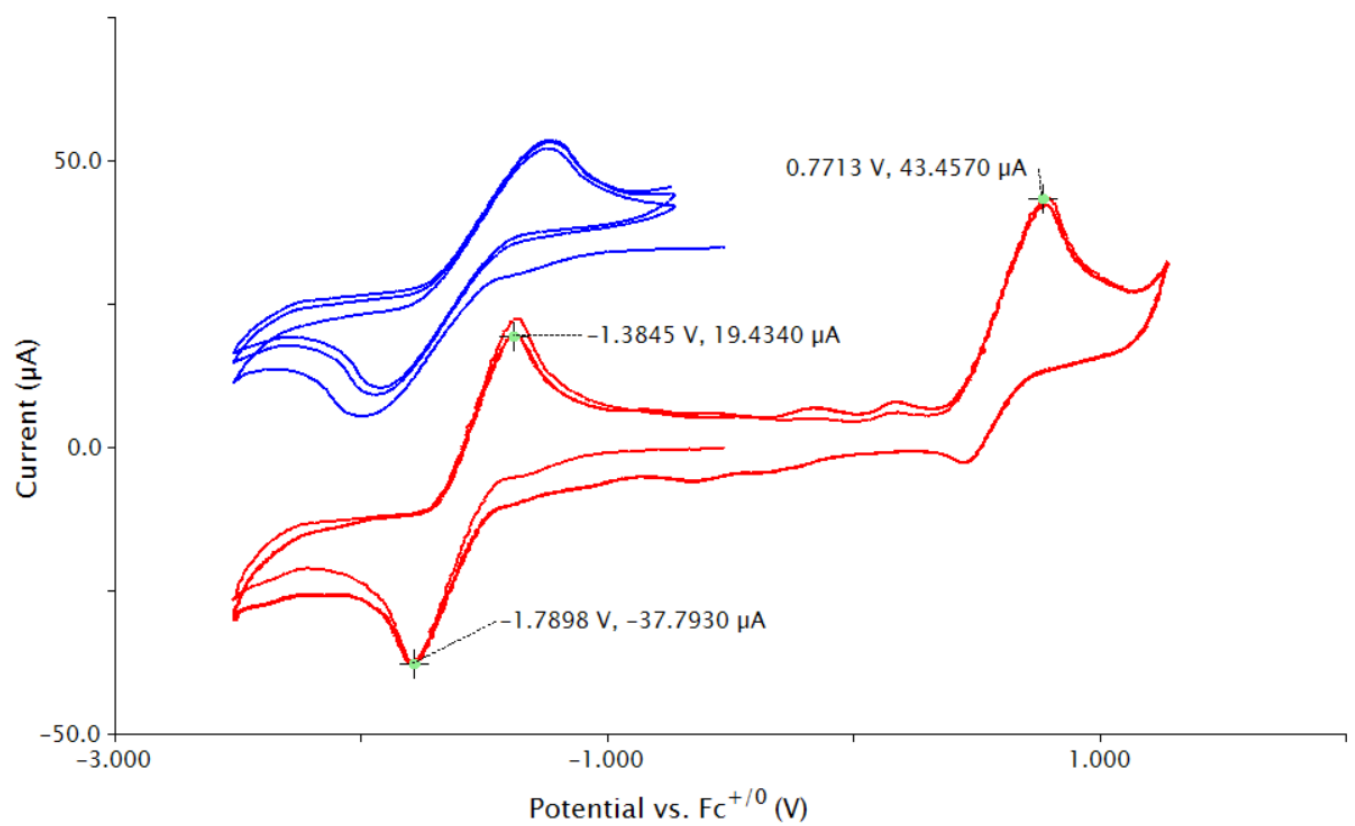

Figure S52. CV data for complex 2.

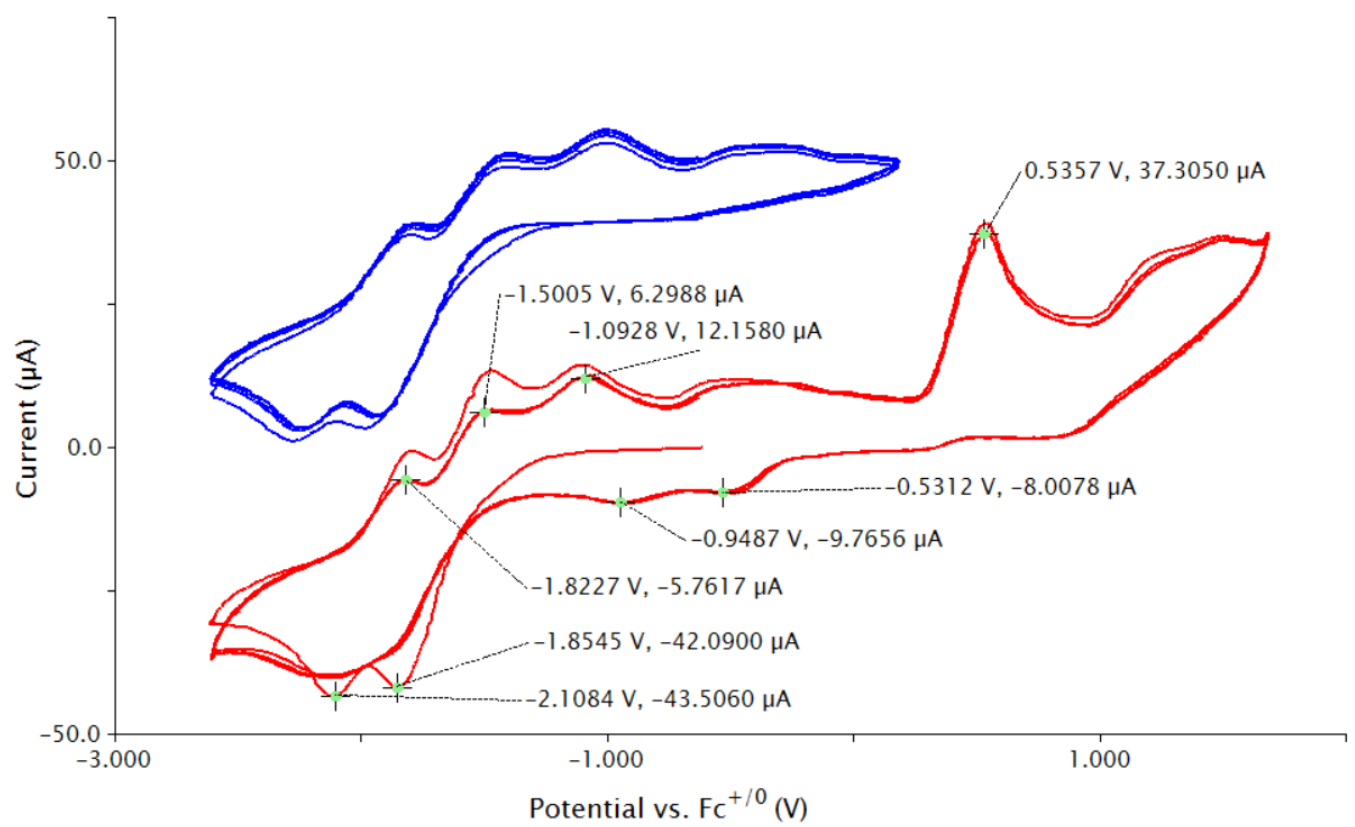

Figure S53. CV data for complex 3. 


\section{Crystallographic Information}

CCDC 1440560-1440565 contain the supplementary crystallographic data for this paper. These data can be obtained free of charge from The Cambridge Crystallographic Data Centre via www.ccdc.cam.ac.uk/data request/cif.

\section{Refinement details}

In each case, crystals were mounted on a glass fiber or nylon loop using Paratone oil, then placed on the diffractometer under a nitrogen stream. Low temperature $(100 \mathrm{~K}) \mathrm{X}$-ray data were obtained on a Bruker APEXII CCD based diffractometer (Mo sealed X-ray tube, $\mathrm{K}_{\alpha}=$ $0.71073 \AA$ ). All diffractometer manipulations, including data collection, integration and scaling were carried out using the Bruker APEXII software.' Absorption corrections were applied using SADABS. ${ }^{10}$ Space groups were determined on the basis of systematic absences and intensity statistics and the structures were solved by direct methods using XS (incorporated into SHELXTL) and refined by full-matrix least squares on $\mathrm{F}^{2}$. All nonhydrogen atoms were refined using anisotropic displacement parameters. Hydrogen atoms were placed in idealized positions and refined using a riding model. The structure was refined (weighted least squares refinement on $\mathrm{F}^{2}$ ) to convergence.

Table S2. Crystal and refinement data for reported complexes.

\begin{tabular}{|c|c|c|c|c|c|c|}
\hline Complex & 2 & 3 & $4-\mathrm{H}$ & $5-\mathrm{H}$ & 6-H & $7-\mathrm{H}$ \\
\hline $\begin{array}{l}\text { empirical } \\
\text { formula }\end{array}$ & $\begin{array}{c}\mathrm{C}_{15} \mathrm{H}_{19} \mathrm{Cl}_{0.06} \mathrm{Ni}_{0 .} \\
{ }_{50} \mathrm{OP}\end{array}$ & $\mathrm{C}_{32} \mathrm{H}_{43} \mathrm{O}_{2.5} \mathrm{P}_{2} \mathrm{Pd}$ & $\mathrm{C}_{69} \mathrm{H}_{88} \mathrm{O}_{4} \mathrm{P}_{4} \mathrm{Pd}_{2}$ & $\begin{array}{c}\mathrm{C}_{35} \mathrm{H}_{48} \mathrm{~F}_{3} \mathrm{ClO}_{6} \mathrm{P}_{2} \\
\mathrm{PdS}\end{array}$ & $\begin{array}{c}\mathrm{C}_{31} \mathrm{H}_{41} \mathrm{~F}_{3} \mathrm{O}_{5} \mathrm{P}_{2} \\
\mathrm{PdS}\end{array}$ & $\begin{array}{c}\mathrm{C}_{32.33} \mathrm{H}_{43.33} \mathrm{AgF}_{3} \\
\mathrm{O}_{5.33} \mathrm{P}_{2} \mathrm{~S}\end{array}$ \\
\hline $\begin{array}{l}\text { formula } \\
\text { wt }\end{array}$ & 277.84 & 636.00 & 1318.07 & 857.58 & 751.04 & 776.20 \\
\hline $\mathrm{T}(\mathrm{K})$ & 100 & 200 & 100 & 100 & 100 & 99.98 \\
\hline $\mathrm{a}, \AA$ & $16.8334(5)$ & $24.0630(6)$ & $12.900(3)$ & $13.040(6)$ & $19.7535(3)$ & $31.152(3)$ \\
\hline $\mathrm{b}, \AA$ & $16.8334(5)$ & $24.0630(6)$ & $13.564(3)$ & $19.284(8)$ & $19.7535(3)$ & $31.152(3)$ \\
\hline c, $\AA$ & 20.9194(8) & $20.9789(6)$ & $19.638(3)$ & $15.679(6)$ & $19.7535(3)$ & $20.2695(18)$ \\
\hline$\alpha, \operatorname{deg}$ & 90 & 90 & $106.655(6)$ & 90 & 110.8200 & 90 \\
\hline$\beta, \operatorname{deg}$ & 90 & 90 & $91.342(6)$ & 108.373(18) & 110.8200 & 90 \\
\hline$\gamma, \operatorname{deg}$ & 90 & 90 & $97.356(5)$ & 90 & 110.8200 & 120 \\
\hline $\mathrm{V}, \AA^{3}$ & $5927.8(4)$ & $12147.4(7)$ & $3258.4(12)$ & $3742(3)$ & $5617.9(3)$ & 17035 \\
\hline $\mathrm{Z}$ & 16 & 16 & 4 & 4 & 6 & 18 \\
\hline cryst syst & Tetragonal & Tetragonal & Triclinic & Monoclinic & Rhombohedral & Trigonal \\
\hline $\begin{array}{l}\text { space } \\
\text { group }\end{array}$ & $\mathrm{P} 2 / \mathrm{ncc}$ & $\mathrm{P}-42{ }_{1} \mathrm{C}$ & P-1 & $\mathrm{P} 12_{1} / \mathrm{n} 1$ & $\mathrm{R} 3 \mathrm{cR}$ & $\mathrm{R} 3 \mathrm{c}$ \\
\hline $\begin{array}{l}\mathrm{d}_{\text {calcd }} \\
\mathrm{g} / \mathrm{cm}^{3}\end{array}$ & 1.245 & 1.391 & 1.344 & 1.515 & 1.332 & 1.362 \\
\hline $\begin{array}{l}\theta \text { range, } \\
\text { deg }\end{array}$ & 1.711 to 36.343 & 1.197 to 37.310 & 1.583 to 37.026 & 1.729 to 36.211 & 2.17 to 29.22 & 2.147 to 30.664 \\
\hline$\mu, \mathrm{mm}^{-1}$ & 0.797 & 0.745 & 0.696 & 0.767 & 0.685 & 0.723 \\
\hline abs cor & $\begin{array}{c}\text { Semi-empirical } \\
\text { from } \\
\text { equivalents }\end{array}$ & $\begin{array}{l}\text { Semi-empirical } \\
\text { from } \\
\text { equivalents }\end{array}$ & $\begin{array}{l}\text { Semi-empirical } \\
\text { from } \\
\text { equivalents }\end{array}$ & $\begin{array}{l}\text { Semi-empirical } \\
\text { from } \\
\text { equivalents }\end{array}$ & $\begin{array}{l}\text { Semi-empirical } \\
\text { from } \\
\text { equivalents }\end{array}$ & $\begin{array}{l}\text { Semi-empirical } \\
\text { from } \\
\text { equivalents }\end{array}$ \\
\hline GOFc & 0.952 & 1.167 & 0.874 & 1.022 & 0.972 & 0.904 \\
\hline $\begin{array}{c}\mathrm{R} 1, \mathrm{a}^{\mathrm{a}} \\
\mathrm{wR} 2^{\mathrm{b}}(\mathrm{I} \\
>2 \sigma(\mathrm{I}))\end{array}$ & $0.0406,0.1039$ & $0.0527,0.0995$ & $0.0521,0.0944$ & $0.0529,0.1286$ & $0.0318,0.0720$ & $0.0475,0.1239$ \\
\hline $\begin{array}{l}{ }^{\mathrm{a}} \mathrm{R} 1 \\
\{\Sigma\end{array}$ & $\begin{array}{l}=\Sigma|| \mathrm{F}_{\mathrm{o}}|-| \mathrm{F}_{\mathrm{c}} \\
\left.\mathrm{w}\left(\mathrm{F}_{\mathrm{o}}^{2}-\mathrm{F}_{\mathrm{c}}{ }^{2}\right)^{2}\right] /\end{array}$ & $\begin{array}{l}\left|/ \sum\right| \mathrm{F}_{\mathrm{o}} \mid \\
1-\mathrm{p})\}^{1 / 2}\end{array}$ & \multicolumn{4}{|c|}{${ }^{\mathrm{b}} \mathrm{wR} 2=\left\{\Sigma\left[\mathrm{w}\left(\mathrm{F}_{\mathrm{o}}^{2}-\mathrm{F}_{\mathrm{c}}{ }^{2}\right)^{2}\right] / \Sigma\left[\mathrm{w}\left(\mathrm{F}_{\mathrm{o}}^{2}\right)^{2}\right]\right\}^{1 / 2} \quad{ }^{\mathrm{c}} \mathrm{GOF}=\mathrm{S}=$} \\
\hline
\end{tabular}




\section{References}

1.Pangborn, A. B.; Giardello, M. A.; Grubbs, R. H.; Rosen, R. K.; Timmers, F. J., Organometallics 1996, 15, 1518-1520.

2.Berliner, M. A.; Belecki, K., J. Org. Chem. 2005, 70, 9618-9621.

3.Eisch, J. J.; Piotrowski, A. M.; Han, K. I.; Kruger, C.; Tsay, Y. H., Organometallics 1985, 4, 224-231.

4.Wariishi, K.; Morishima, S.; Inagaki, Y., Org. Process Res. Dev. 2003, 7, 98-100.

5.Zhang, J. X.; Cui, Y. P.; Wang, M. L.; Liu, J. Z., Chem. Commun. 2002, 2526-2527.

6.Horak, K. T.; Lin, S.; Rittle, J.; Agapie, T., Organometallics 2015, 34, 4429-4432.

7.Fulmer, G. R.; Miller, A. J. M.; Sherden, N. H.; Gottlieb, H. E.; Nudelman, A.; Stoltz, B. M.; Bercaw, J. E.; Goldberg, K. I., Organometallics 2010, 29, 2176-2179.

8.Velian, A.; Lin, S. B.; Miller, A. J. M.; Day, M. W.; Agapie, T., J Am Chem Soc 2010, 132, 6296-6297.

9.APEX2, Version 2 User Manual, M86-E01078, Bruker Analytical X-ray Systems, Madison, WI, June 2006.

10.Sheldrick, G. M. "SADABS (Version 2008/1): Program for Absorption Correction for Data from Area Detector Frames”, University of Göttingen, 2008. 